\title{
Blue light-dependent development of the filamentous fungus Aspergillus nidulans
}

\author{
Dissertation \\ zur Erlangung des Doktorgrades \\ der Mathematisch-Naturwissenschaftlichen Fakultäten \\ der Georg-August-Universität zu Göttingen \\ vorgelegt von \\ Özgür Bayram \\ aus Sakarya / Türkei
}

Göttingen 2007 
This Ph.D work was performed in the group of Prof. Dr. Gerhard H. Braus at the Department of Molecular Microbiology and Genetics at the Institute of Microbiology and Genetics, Georg-August-University, Göttingen.

A chapter of this Ph.D thesis has been published and two other chapters have been submitted for publication.

Bayram, Ö., Biesemann, C., Krappmann, S., Galland, P., and Braus, G.H. (2007). Fungal CryA: the missing link between cryptochromes and photolyases. (submitted for publication).

Bayram, Ö., Krappmann, S., Seiler, S., Vogt, N., and Braus, G.H. (2007). Neurospora crassa $v e-1$ affects asexual sporulation. Fungal Genet Biol (in press, doi: 10.1016/j.fgb.2007.06.001)

Bayram, Ö., Krappmann, S., Helmstaedt, K., Valerius, O., Ni, M., Yu, J-H., and Braus, G.H. (2007). The velvet complex coordinates light, fungal development and secondary metabolism. (under revision).

D7

Referent $\quad$ : Prof. Dr. G. H. Braus

Koreferent : Priv.-Doz. Dr. Sven K. Krappmann

Tag der mündlichen Prüfung : 01.11.2007 
To my parents, Bayram Ali Bayram and Hatice Bayram 


\section{Acknowledgements}

My special thanks to Prof. Dr. Gerhard Braus for his supervision and his support for this thesis with endless inspiration.

Special thanks to Dr. Sven Krappmann for his excellent bench supervision and friendly support outside the lab. He taught me a lot about cloning and molecular genetics.

I would like to thank Prof. Dr. Oliver Einsle for the oral biochemistry exam. I am also thankful to Dr. Kerstin Helmstaedt for her co-operative approach in the TAP tag project. Dr. Stephan Seiler for his collaboration in the Neurospora crassa velvet project, Dr. Oliver Valerius for his helpful discussions as well as mass spectrometry identification of velvetinteracting proteins. Christoph Bieseman for his excellent work in the Aspergillus cryA project. Additionally I would like to thank Prof. Dr. Paul Galland (Marburg University) for his advice in photobiology and his very valuable comments on the Aspergillus cryA project. Here, I would like to mention Verena Große especially from whom I learned a lot at the beginning of my Ph.D study. Of course, I do not forget to thank Fatih Sari as my very precious friend and for his collaboration in Aspergillus imeB project along with Dr. Stefan Irniger.

I am also thankful to Nirmala Padmanabhan for proof reading this thesis.

My thanks to all present and former members of the Aspergillus lab, Dr. Oliver Draht, Christoph Sasse, Karen Laubinger, Dr. Anne Krappmann, Nico Vogt, Verena Pretz, Maria Meyer and Dr. Lars Fichtner and others for providing a friendly atmosphere, our team of secretaries, Heidi Northemann and Nicole Scheiter and our technical team members, Patrick Regin for building the illumination chamber and Gerd Birke for his friendly approach.

I do not forget to mention my former supervisor Prof. Dr. Sezai Türkel (Uludag University, Turkey) who gave me invaluable advice and supported me a lot at the beginning and during my Ph.D study.

Finally, I want to thank my supportive wife Özlem Sarikaya Bayram and my parents who have always supported me and all my friends for their general support. 


\section{Table of contents}

Abbreviations 1

Summary 3

Zusammenfassung 5

Chapter 1:

$\begin{array}{ll}\text { Introduction } & 7\end{array}$

1. Ecological importance of fungi $\quad 7$

$\begin{array}{lll}1.1 & \text { Environmental factors affecting fungal growth } & 7\end{array}$

1.2 Effect of illumination on fungal development 8

2. Development in ascomycetes 11

$\begin{array}{ll}2.1 & \text { Fruiting body formation } \\ \end{array}$

$\begin{array}{ll}2.2 & \text { Requirements of fruit body formation } \\ \end{array}$

$\begin{array}{lll}2.3 & \text { Effect of environmental factors on sexual development } & 13\end{array}$

2.4 Genetic elements governing fruit body formation 15

2.5 velvet (veA) gene of Aspergillus nidulans 19

3. Secondary metabolism 20

$\begin{array}{ll}\text { Aim of the work } & 23\end{array}$

$\begin{array}{ll}\text { References } & 25\end{array}$

Chapter 2:

More than a repair enzyme: Aspergillus nidulans photolyase-like CryA is a regulator of sexual development $\quad 35$

$\begin{array}{ll}\text { Abstract } & 35\end{array}$

$\begin{array}{ll}\text { Introduction } & 36\end{array}$

Materials and Methods $\quad 39$

$\begin{array}{ll}\text { Results } & 46\end{array}$

Discussion $\quad 59$

References $\quad 63$ 
Chapter 3:

Neurospora crassa ve-1 affects asexual conidiation

$\begin{array}{ll}\text { Abstract } & 69\end{array}$

$\begin{array}{ll}\text { Introduction } & 70\end{array}$

$\begin{array}{ll}\text { Materials and Methods } & 73\end{array}$

$\begin{array}{ll}\text { Results } & 77\end{array}$

$\begin{array}{ll}\text { Discussion } & 85\end{array}$

$\begin{array}{lr}\text { References } & 89\end{array}$

Chapter 4:

The velvet complex coordinates light, fungal development and secondary $\begin{array}{lr}\text { metabolism } & 95\end{array}$

$\begin{array}{ll}\text { Abstract } & 95\end{array}$

$\begin{array}{ll}\text { Introduction } & 96\end{array}$

$\begin{array}{lr}\text { Materials and Methods } & 98\end{array}$

$\begin{array}{ll}\text { Results } & 109\end{array}$

$\begin{array}{ll}\text { Discussion } & 121\end{array}$

$\begin{array}{lr}\text { References } & 125\end{array}$

$\begin{array}{ll}\text { Conclusions } & 131\end{array}$

$\begin{array}{ll}\text { Fungal CryA: the missing link between cryptochromes and photolyases } & 131\end{array}$

$\begin{array}{ll}\text { Velvet proteins are highly conserved in ascomycetous fungi } & 132\end{array}$

The Velvet complex : A core element that regulates the light-dependent development and secondary metabolism in A. nidulans 132

$\begin{array}{lr}\text { References } & 135\end{array}$

$\begin{array}{ll}\text { Curriculum Vitae } & 139\end{array}$ 
Abbreviations

AF

BIFC

CBP

CPD

CRY

CRY-DASH

DTT

EDTA

EGTA

EYFP

FAD

GFP

HPLC

NES

NLS

PABA

PEST

PMSF

PCR

RFP

$\mathrm{SC}$

ST

SDS

TAP

TCA

TEV

UV

$\mathrm{Y} 2 \mathrm{H}$

XCorr

WC

$\mathrm{Wt}$ aflatoxin

bimolecular fluorescence complementation

calmodulin binding peptide

cyclobutane pyrimidine dimer

cryptohrome

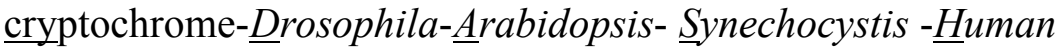

$D L$-dithiothreitol

ethylenediaminetetraacetic acid

ethylene glycol tetraacetic acid

enhanced yellow fluorescence protein

flavin adenin dinucleotide

green fluorescent protein

high performance liquid chromatography

nuclear export signal

nuclear localization signal

paraminobenzoic acid

pro, glu (or a spp), ser, thr motif

phenylmethanesulfonylfluoride

polymerase chain reaction

red fluorescent protein

synthetic complete

sterigmatocystin

sodium dodecyls sulfate

tandem affinity purification

trichloroacetic acid

tabacco etch virus

ultraviolet

yeast two-hybrid

cross-correlation

white collar

wild type 


\section{Summary}

Numerous studies have revealed that fungal development and the production of secondary metabolites are linked although the molecular mechanisms are yet unknown. Light seems to be one of the environmental triggers for the coordination of these processes. Here, the lightcontrolled synchronization of the formation of sexual fruitbodies (cleistothecia) and secondary metabolism was analysed in the filamentous model fungus Aspergillus nidulans.

The light response of the fungus was addressed by analyzing a cryptochrome-like gene product as putative blue light receptor. A. nidulans carries a single gene in its genome, termed $\operatorname{cry} A$, with the capacity to encode a cryptochrome/photolyase-like protein. We aimed at the characterization of cryA and its product as a first fungal cryptochrome. Detailed interpretation of experimental results and findings revealed that the A. nidulans CryA is a unique protein among the cryptochrome/photolyase-like protein family because it is a dual function protein which regulates gene expression in A. nidulans: it repairs UV-induced DNA damage both in a UV repair defective E.coli and in A. nidulans. Expression experiments revealed that it regulates a novel transcriptional feedback loop including the so-called light-dependent velvet regulatory protein. Expression of the veA gene is affected by CryA activity.

The $A$. nidulans velvet $A$ gene, veA, is an essential component of the light-dependent sexual development regulation whose function is inhibited in day light (red light) and actived in the absence of light (darkness). veA represses asexual development and promotes sexual fruit body formation in A. nidulans. Here, an orthologue of velvet in the heterothallic fungus Neurospora crassa, and showed that the N.crassa velvet, ve-1, is conserved structurally and functionally. It also regulates fungal development resulting in stunted hyphal growth and increased asexual conidiation of mutant strains.

In addition to its role in sexual development in $A$. nidulans, ve $A$ is also involved in the regulation of secondary metabolism, since deletion of $v e A$ results in a complete loss of cleistothecia formation and the loss of sterigmatocystin production as well as downregulation of the expression of genes responsible for penicillin biosynthesis. The molecular mechanism of this connection between light control and secondary metabolism is yet unknown and has been addressed in this work, too. For this purpose, interaction partners of VeA were identified using a generic Tandem Affinity Purification (TAP) tag approach. It turned out that VeA is a part of a trimeric protein complex constituted by VelB, VeA and the putative LaeA methyl transferase, a master regulator of secondary metabolism. This newly defined "velvet complex" appears to regulate both light-dependent sexual development and secondary metabolism in A. nidulans. 


\section{Zusammenfassung}

Zahlreiche Studien belegen, dass in Pilzen Entwicklungsprozesse und die Produktion von Sekundärmetaboliten gekoppelt sind, die molekulare Mechanismen dieses Zusammenhangs sind jedoch bislang unbekannt. Dabei scheint Licht ein Umweltfaktor zu sein, der diese Prozesse bedingt. In dieser Arbeit wurde die lichtvermittelte Synchronisation der Fruchtkörperbildung mit dem Sekundärmetabolismus des pilzlichen, filamentösen Modelorganismus' Aspergillus nidulans erforscht.

Die Lichtantwort dieses Pilzes wurde durch Analyse eines Cryptochrom-artigen Genprodukts als potentiellen Blaulichtrezeptor untersucht. Das Genom von A. nidulans beinhaltet ein einziges, als cryA bezeichnetes Gen, das für ein Cryptochrom- bzw. Photolyaseartiges Protein kodieren könnte. Eingehende experimentelle Untersuchungen zeigten, dass CryA von $A$. nidulans ein einzigartiges Protein der Cryptochrom/Photolyase-ähnlichen Proteinfamilie darstellt, da es aufgrund seiner dualen Funktionsweise die Genexpression in A. nidulans reguliert: UV-induzierte DNA-Schäden werden von CryA sowohl in einem reparaturdefizienten E. coli-Stamm als auch in A. nidulans behoben. Expressionstudien zeigten, dass es darüber hinaus eine bislang unbekannte Transkriptionsregulationsschleife reguliert, die das lichtabhängige Regulatorprotein velvet beinhaltet. Die Expression des kodierenden veA-Gens wird durch die CryA-Aktivität beeinflusst.

Das velvet $A$ (veA)-Gen von $A$. nidulans ist eine essentielle Komponente der Regulation der lichtabhängigen sexuellen Entwicklung, deren Funktion durch Tages- bzw. Rotlicht inhibiert und in Dunkelheit aktiviert wird. veA reprimiert in A. nidulans die asexuelle Entwicklung und unterstützt die Bildung sexueller Fruchtkörper. Im Rahmen dieser Arbeit wurde ein Orthologes in dem heterothallischen Pilz Neurospora crassa identifiziert, und es konnte gezeigt werden, dass das velvet-Gen aus $N$. crassa, ve-1, sowohl strukturell als auch funktionell konserviert ist. Darüber hinaus reguliert es die Pilzentwicklung, veranschaulicht durch das Erscheinungsbild (verkürzte Hyphen, vermehrte asexuelle Sporulation) entsprechender Mutanten.

Über seine Rolle innerhalb der sexuellen Differenzierung in A. nidulans hinaus ist VeA auch an der Regulation des Sekundärstoffwechsels beteiligt. Deletion von veA führt zur vollständigen Blockade der Kleistothezienentwicklung, der Sterigmatocystinproduktion und zu einer verminderten Expression von Genen, die zur Penizillinbiosynthese benötigt werden. Die molekularen Grundlagen dieser Verbindung von Lichtregulation und Sekundärmetabolismus sind bislang unbekannt und sollten innerhalb dieser Arbeit ebenfalls untersucht werden. Hierfür wurden Interaktionspartner von VeA mittels des sog. Tandem-AffinityPurification (TAP)-tag-Ansatzes identifiziert. Dabei stellte sich heraus, dass VeA in einem trimeren Proteinkomplex vorliegt, bestehend aus VelB, VeA und der potentiellen LaeAMethyltransferase, einem Hauptregulator des Sekundärmetabolismus. Dieser hier erstbeschriebene „Velvetkomplex” reguliert anscheinend sowohl die lichtabhängige sexuelle Entwicklung als auch den Sekundärmetabolismus von $A$. nidulans. 


\section{Chapter 1}

\section{Introduction}

\section{Ecological importance of fungi}

The fungi are a diverse group with an estimated 1.5 million member having an immense effect on human affairs, the world ecosystem (Borkovich et al., 2004). They produce numerous economically important compounds, such as enzymes, antibiotics and secondary metabolites, some of which are either carcinogenic or anti-therapeutic (Wainright, 1992). Along with bacteria, fungi are the major decomposers in terrestrial ecosystems, they process the decaying material and therefore make a great contribution to the recycling of organic material in the earth. They also play a critical role in biogeochemical cycles and in food webs. Fungi often have important symbiotic relationships with other organisms. Mycorrhizal symbiosis between plants and fungi is particularly important; over $90 \%$ of all plant species establish some kind of mycorrhizal relationship with various fungi and are dependent upon this relationship for survival (Smith, 1997). Sometimes fungi can be deleterious causing infections of humans, animals and plants and these infections result in billions of dollars of loss every year (Agrios, 1997; Latge, 1999). Some members of fungi are used as food such as mushrooms (Basidiomycetes). Some members of ascomycetes such as Saccharomyces cerevisiae (yeast ascomycetes) and Aspergillus oryzae (filamentous ascomycetes) are utilised for the fermentation of alcoholic beverages (beer, wine, champaign etc), pastry products by bakers and the production of the traditional japanese drink, sake respectively. Fungi are also a very important group as model organisms to understand the biology, physiology, genetics, and biochemistry of a eukaryotic cell. In summary fungi are an indispensable component of our ecosystem and they play an essential role for the sustenance of life on the earth.

\subsection{Environmental factors affecting fungal growth}

Fungi, like many other living organisms, need various external environmental parameters for growth, differentiation and reproduction. In some Basidiomycetes, the influence of environmental factors on fruiting body development is characterized at the physiological level (Kues and Liu, 2000). Despite the fact that there are many factors for fungal development including nutrition accessibility, temperature, $\mathrm{pH}$, minerals, chemicals, aeration and light, fungi require different parameters depending on the stage of fungal development, namely, 
vegetative, asexual sporulation and sexual fruiting body formation. The most important factors are the nutritional compounds which provide carbon (mono-, di- and polysaccharides, glycerol, acetate) and nitrogen sources (nitrate, ammonium, glutamate, amino acids, peptides) for the synthesis of macromolecules such as nucleic acids, lipids and supporting the complex differentiated structures like fruiting bodies. Along with nutrition, temperature, $\mathrm{pH}$ and light are very important environmental signals that control their growth rate and morphogenesis. Temperature is a physical parameter that affects enzymatic activity and the metabolism of a fungus. In addition, acidity and alkalinty of the environment are crucial factors and most of the fungi prefer an optimal $\mathrm{pH}$ of 7.0 or acidic $\mathrm{pH}$ in order to balance their homeostasis and maintain growth. Solar energy as a heat and as a light source provides an essential environmental signal. The presence or absence of light results in diverse cellular responses and morphological formations.

\subsection{Effect of illumination on fungal development}

Solar light is essential as an energy source for photosynthetic organisms such as plants and photosynthetic bacteria to maintain photosynthetic reactions. Light can also be recognized as an environmental signal by numerous non-photosynthetic organisms such as animals, fungi or non-photosynthetic bacteria. Different types of light absorbing molecules receive the various spectra of light. The molecular mechanisms of light perception seems to be well-conserved during evolution from archaea and fungi to humans (Sancar, 2000; Fankhauser and Staiger, 2002; Idnurm and Heitman, 2005; Purschwitz et al., 2006; Montgomery, 2007). There are mainly three types of light receptor in fungi: phytochromes (red light receptors, $665 \mathrm{~nm}$ ), cryptochromes (blue light sensors, 390-500 nm) and phototrophins (blue light sensors, 390$500 \mathrm{~nm})$.

Light carries another meaning for fungi most of which are sessile and saprophytic. Fungi have to produce spores in order to survive in nature because most fungi grow either on the surfaces or under the subtsrate. For efficient sporulation to occur, the fungi must sense an air-substrate interface. There are many consequences of light on fungi: promotion of asexual conidiation, entraining the circadian clock, induction of carotenoid pigment synthesis, regulation of secondary metabolism, inhibition of sexual development. The term, "fungus eye”, seems to be appropriate to describe the broad range of light responses of fungi and the corresponding light receptors (Fig. 1.) There are only two light responding mechanisms which have been studied but are not yet fully understood, i.e., response to blue and red light (Liu et al., 2003; Purschwitz et al., 2006). 
A

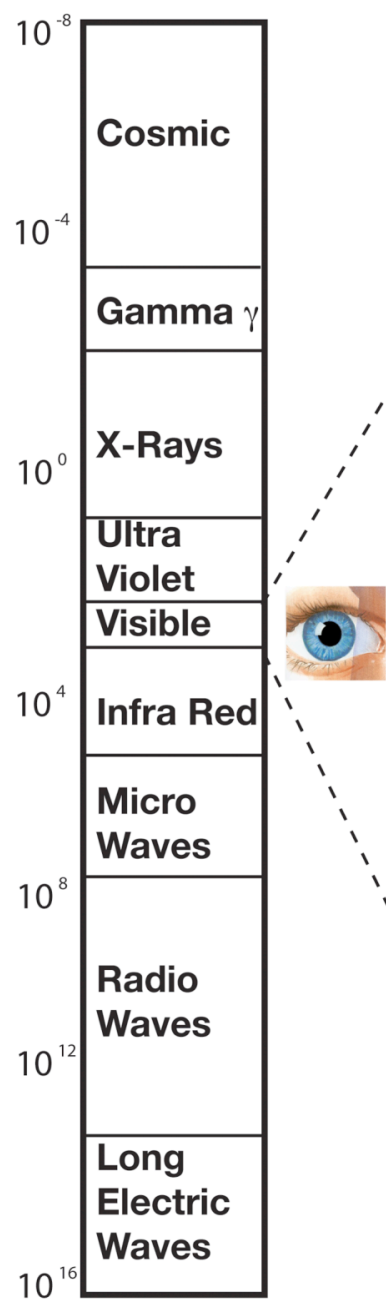

B

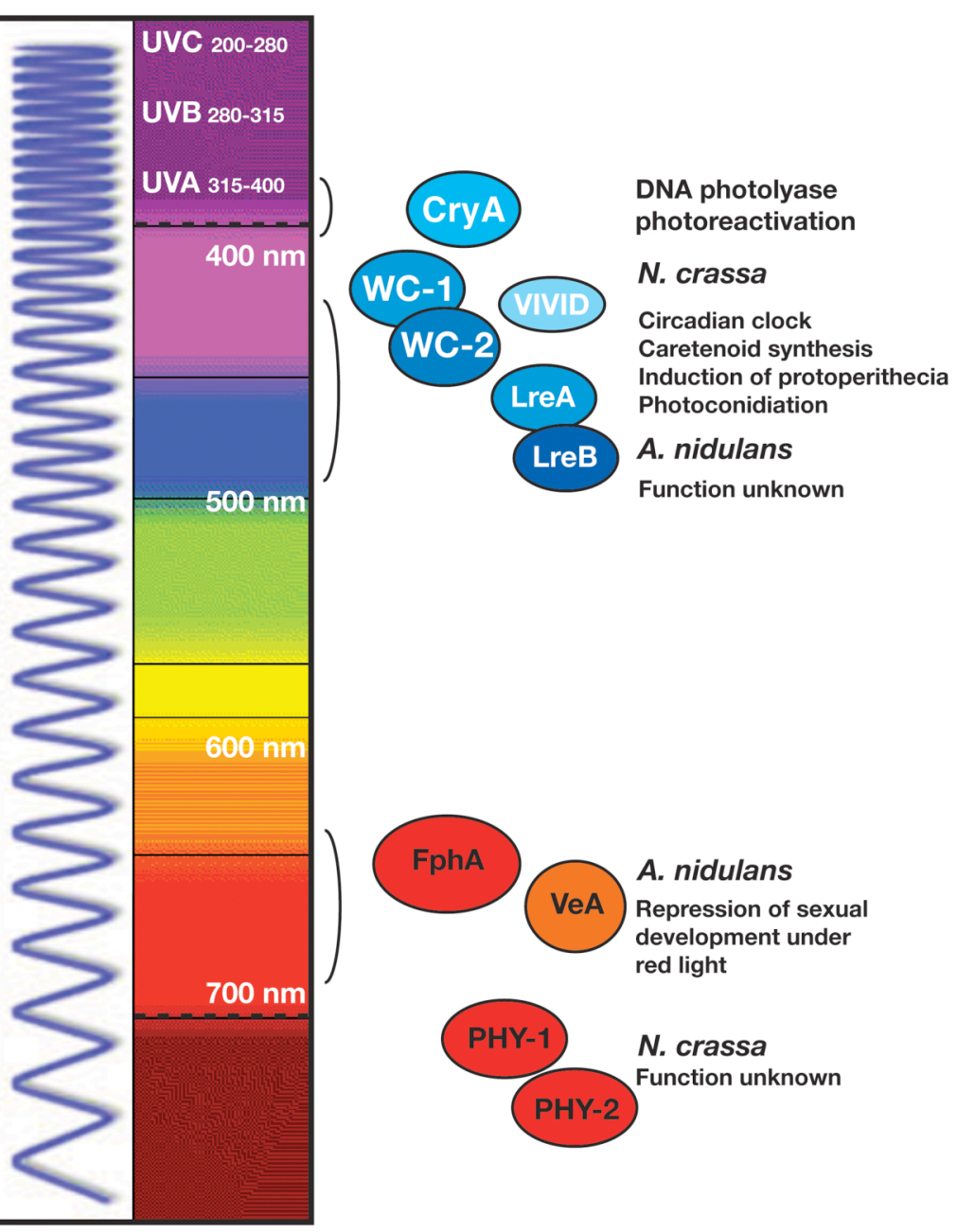

Figure 1: Physical light spectra and fungal receptors that sense visible light. (A) Depiction of solar radiation range from the biggest to the smallest wavelength (B) The visible light spectra (white light) contains wavelengths ranging from blue to far red. A small portion of UV is also depicted. The major fungal light receptors that sense the various wavelengths of the visible sprectra: WHITE COLLAR homologues from different fungi including $N$. crassa $\mathrm{WC}-1$ and WC-2 receive the blue light spectra and UV light to some extent and regulates the circadian rhythm, carotenoid synthesis, induction of protoperithecia and photoinduction (Ballario et al., 1998). Phytochrome protein FphA (Blumenstein et al., 2005) in A. nidulans acts over the velvet regulatory protein (Kim et al., 2002), acquires the red light signal. The receptor function of fungal cryptochromes and opsin homologues (nop-1 N. crassa (Bieszke et al., 1999), nopA A. nidulans) are yet unknown.

Blue light receptors in fungi can be seperated into two major groups: the cryptochromes and the phototropins. Cryptochromes demonstrate high sequence homologies to DNA photolyases, but they have lost the capability of repairing DNA pyrimidine dimers in response to UVA illumination. On the other hand, they have gained different abilities such as 
gene regulation, the entrainment of circadian rhythm and blue light dependent cellular responses. However, fungal cryptochromes have not been thoroughly characterized so far.

The second group of fungal blue light sensors are the phototrophins. WHITE COLLAR-1 and -2 (WC-1 and WC-2) of Neurospora crassa are well-characterised phototropin-like proteins. They govern the main light responses such as induction of protoperithecia, caretenoid biosynthesis, entrainment of circadian clock, induction of hyphal growth, asexual spore formation in response to blue light or UVA light spectrum (Liu et al., 2003). Detailed analyses of the WC-1 and WC-2 protein sequences disclosed that both proteins are GATA-type zinc-finger transcription factors that contain the Per-Arnt-Sim (PAS) domain (Ballario et al., 1998). WC-1 forms a complex with the PAS domain of WC-2 (Talora et al., 1999; Cheng et al., 2002) and hetero dimer plays a role as the transcriptional activator of light regulated genes. WC-1 domain of the complex binds to light-response elements (LRE) in the promoter of the frequency gene $(f r q)$ and confers light dependent transcriptional regulation. Both proteins WC-1 and WC-2 are nuclear proteins and are phosphorylated in a light-dependent fashion which does not affect their localization (Schwerdtfeger and Linden, 2000). WC-1/WC-2 complex controls the expression of frq gene which is the central component of the circadian clock. The phosphorylation of $\mathrm{WC}-1$ by protein kinase $\mathrm{C}$ (PKC) regulates the concentration of the protein and hyperphosphorylation alters its binding ability to the target promoters, which is vital for photoadaptation (He and Liu, 2005).

Another blue light receptor found in fungi is called VIVID and was shown to consist of one LOV (Light-oltage- $\underline{\text { Oxygen }}$ ) domain and to bind non-covalently to the flavin (FAD or FMN) (flavin adenin dinucleotide, flavin mononucleotide) in $N$. crassa. VIVID was speculated to sense alterations in light intensity and to involve in the modulation of the circadian clock (Elvin et al., 2005). Moreover VIVID's expression is regulated by the WC1/WC-2 compex. The shift in the frame of the circadian rhythm stems from the mutations in the $v v d$ gene. WC-like blue light receptors in the ascomycetes $T$. atroviridae (Casas-Flores et al., 2004), the rice blast fungus Magnaporthe oryzae, and A. nidulans have been described (Purschwitz et al., 2006). It has been recently published that the phototropism defective Phycomyces blakesleeanus bears mutations in madA gene that is responsible for photoropism and encodes a WC-1 photoreceptor (Idnurm et al., 2006).

The second prominent but less-studied light response observed in fungi is the red light response. Phytochromes, which are answerable for the red/far-red light-related responses from fungi to plants, utilize a linear tetrapyrrole as the chromophore (Rockwell et al., 2006). Phytochromes undergo a switch bewteen two different conformations: a red light-absorbing 
(Pr) form and a far-red light absorbing (Pfr) conformation. Phytochormes in general have more or less conserved motifs including an N-terminal input domain comprising PAS, GAF (cGMP-specific phosphodiesterases; cyanobacterial adenylate cyclases; formate hydrogen lyase) and PHY (phytochrome) subdomains and a C-terminal modulator domain. Several red light effects have been shown in fungi: in A. nidulans, red light triggers asexual conidiation while inhibiting sexual fruit body formation. The corresponding gene was identified as the $A$. nidulans phytochrome encoding gene $f p h A$. Deletion of $f p h A$ results in a fungus blind to red light and the disappearance of the inhibitory effect of red light on sexual development (Blumenstein et al., 2005). In the true slime-mould Physarum polycephalum, the fragmentation of the plasmodium and sporulation can be induced by far-red light and the induction of sporulation can be suppressed by a red light pulse (Starostzik and Marwan, 1995; Kakiuchi et al., 2001). Only two model ascomycetes A. nidulans and N. crassa were investigated for their red light response at the molecular level. N. crassa in contrast to $A$. nidulans possesses two phytochrome encoding genes phy-1 and phy-2. Disruption of both phytochrome encoding genes uncovered no obvious phenotype (Froehlich et al., 2005). Thus the role of phytochromes still remains elusive in this organism. Another effect was observed in strains that bear the mutant $v e A l$ allele of the $v e A$ gene which is the light-dependent sexual development regulator of A. nidulans (Mooney et al., 1990; Mooney and Yager, 1990; Kim et al., 2002). veAl laboratory starins are insensitive to red light and reduced in fruit body formation (see page 19). It was suggested that phytochrome FphA physically interacts with VeA (Purschwitz et al., 2006).

Opsins, which are the important component of animal eyes and the archaeal energy conservation mechanism, have seven transmembrane helices and are capable of transmitting light signals to optical nerves. Deletion of the opsin homologue encoding gene nop- 1 of $N$. crassa did not reveal any apparent phenotype indicating the yet unknown functions of fungal opsins (Bieszke et al., 1999).

\section{Development in ascomycetes}

Vegetative growth in ascomycetes, as in other filamentous group of fungi, starts with the germination of a spore that could be either a mitotically derived conidiospore or a meiotically produced ascospore. Germination of spores leads to the establishment of tubular hyphae that grow in a polar manner by apical extension of the Spitzenkörper and give rise to branches that organize into a network of interconnected cells named as a mycelium. Most of the time the mycelium forms a radially symmetric colony which expands indefinitely at a constant rate. At first glance, though the vegetative mycelium seems to be quite similar, in fact there are several diverse cells or different hyphae that have special functions such as in the acquisition of nutrients from the environment and in determining the precise time of differentiation to yield 
reproductive structures (Adams et al., 1998). The competence time indicates the time of which hypha commits to form the differentiated reproductive structures. This competence time varies depending on the organism and environmental conditions. However most of the time, it is about 10 hours after inoculation. For differentiation in most cases, a solid surface is required and as long as an ascomycetous organism grows in a submerged culture, it cannot differentiate into reproductive structures and only stays in the vegetative phase. However, these hyphae as soon as they are transferred on to a solid surface, immediately begin to establish reproductive organs because they are already competent for further development.

After vegetative growth, asexual spore-bearing conidiophores are established and these conidiophores generate conidia which are named differently depending on the group. $A$. nidulans produces only one type of asexual spores called conidia while $N$. crassa generates two types of spores, both multinucleate macrospores that originate from the conidiophores and single nucleus bearing microspores that directly stem from the growing hyphae. Many gene products, which participate in the spatial and temporal regulation of the asexual developmental programme, act as various types of transcription factors (Springer and Yanofsky, 1989).

Once asexual sporulation is complete, then sexual development commences with the formation of specialized cell types that are precursors of sexual structures. Sexual life cycle of ascomycetes can be either heterothallic (self-sterile) or homothallic (self-fertile). Depending on the group, sexual development might require the presence of an opposite mating type to be able to develop sexually. After fusion of similar nuclei as in homothallic species as different nuclei as in heterothallic species, the resulting zygote undergoes meiosis and consecutive mitosis. Finally sexually formed ascospores are produced in the ascus (sac like stuctures) inside the complex fruit bodies. However, there are many Aspergilli that do not have any known sexual cycle, e.g., A. fumigatus, A. flavus and A. niger.

\subsection{Fruiting body formation}

A typical feature of many ascomycetes as a consequence of their sexual reproduction cycle is a sac-like bag that is named the ascus (Gr. askos = sac, goat skin). The ascus is filled with meiotically formed ascospores (Braus et al., 2002b). The number of ascospores inside the ascus varies between one and 1000 depending on the species. In the classification of ascomycetes, fruit bodies have a significant place as they are characteristic for each group. There are three types of fruit bodies in ascomycetes: (1) Cleistothecium (closed), (2) Perithecia (closed with a neck) and (3) Apothecium (open).

Aspergillus nidulans produces closed fruit bodies (cleistothecia) that consist of meiotically generated purple-red-coloured ascospores inside the asci. A. nidulans is the only member of Aspergilli spp which has ability to propagate sexually. Sexual development characteristically begins after conidiophore differentiation. In fungi, two different mating 
types, an antheridium cell (the male sexual structure) and an ascogonium (the female sexual structure) fuses to give rise to a dikaryotic hypha. A. nidulans is a homothallic ascomycete. Therefore sexual development takes place either by mating of two strains or by selfing. $A$. nidulans possesses both mating type genes in its genome. In A. nidulans nests, dikaryotic hyphae are formed by fertilization events and afterwards undergo an extended series of coordinated cellular and nuclear divisions. The surrounding mycelium is subject to differentiation in order to form the cleistothecial envelope (Sohn and Yoon, 2002). Nests are covered with specialized thick-walled cells called Hülle cells because of their protective and nursing roles in sexual development. Inside the nests, first forms of ascogenous hyphae are established, which are dikaryotic and later give shape to the so-called croziers. Two nuclei are trapped in the topmost crozier cell by a series of nuclear divisions. In every single crozier, a nuclear fusion event (karyogamy) happens by forming a diploid nucleus and this short-life zygote immediately undergoes meiosis and four nuclei come into being. After meiosis, one round of mitosis generates eight nuclei that are seperated from each other by membranes. Another round of mitotis gives rise to eight binucleate ascospores in an octad of an $A$. nidulans ascus. On average, the size of a mature cleisothecia is about $200 \mu \mathrm{m}$ and generally contains approximately 80.000 viable ascospores. The colour of ascospores is red due to the accumulation of a distinctive red pigment named asperthecin.

\subsection{Requirements of fruit body formation}

Sexual development is controlled by many environmental and internal factors. As many other organisms, A. nidulans grows and reproduces within a certain microambience that shows some fluctuations and changes in its properties swiftly in time and space. The organisms have to decide whether to commence a certain programme based on the enviromental circumstances. Considering the fruit body formation, the fullfillment of a number of optimal conditions are required before the sexual cycle of $A$. nidulans can be initiated and successfully completed. Essential environmental and internal factors of fruit body formation are shown in Fig. 2 (Fig. 2 was adapted from (Braus et al., 2002b)).

\subsection{Effect of environmental factors on sexual development}

Light is a crucial parameter for fruit body formation of $A$. nidulans. Incubation of $A$. nidulans wt strain in the darkness leads to more number of fruit bodies while incubation in the light results in less number of fruit bodies. Conidiation is induced by red light pulse $(680 \mathrm{~nm})$ and fruit body formation is promoted by far-red light pulse $(740 \mathrm{~nm})$ (Mooney and Yager, 1990). 
On the other hand, in $N$. crassa, blue light is the prevailing signal to induce various developmental processes including protoperithecia formation (Harding and Melles, 1983; Degli-Innocenti et al., 1984). Another factor that affects fruitbody formation in combination with light is air exchange. Prevention of air exhcange stimulates an increase in the number of fruit bodies. Approximately 2000 fruit bodies per $10 \mathrm{~mm}^{2}$ are produced by A. nidulans, which is stimulated by wrapping the plates. It is speculated that this is due to a reduction of $\mathrm{CO}_{2}$ content of the air that stalls fruit body development. Including fruit body formation, all differentiation events depend on a proper surface. Differentiation of a mycelium, which does not take place under submersed culture even after prolonged incubation time, necessarily demands of a solid surface. This medium/air interface can be either solid or liquid for proper differentiation. In some extreme conditions such as poor amount of nitrogen source in liquid media may stimulate the hyphae to conidiate but amount is not as much as it is on the surface. Also overexpression of either sexual or asexual regulators might cause the propagation of differentiation programmes independent of surface (Adams et al., 1988; Han et al., 2001; Kim et al., 2002).

In addition to environmental conditions, the nutritional content of the growth media has an important effect on the cleistothecia formation. Limitation of carbon or nitrogen source inhibits sexual development or results in malformations in fruit body formation. Limited carbon source presumably inhibits fruit body formation through a lack of $\alpha$-1,3-glucan components indispensible for the establishment of the cleistothecial cell walls or as energy storing molecules. The availability of N-source such as ammonium, nitrate, amino acids, peptides, translational precursors of proteins affects developmental programs of $A$. nidulans. Imbalance in the nitrogen source influences fruit body formation negatively reducing the number and sizes of cleistothecia (Serlupi-Crescenzi et al., 1983). An additional environmental factor is so-called psi factor (precocious sexual inducer) that is an endogenous mixture of hydroxylinoleic acid moieties (Champe et al., 1987; Champe and el-Zayat, 1989). Application of psi factor on the A. nidulans cultures stimulate early sexual development by inhibiting asexual conidiation. The exact mechanism of psi was speculated to be at the level of the fungal cell membrane and induce the changes in the membrane properties leading to special hyphal fusions yielding the dikaryon. The psi could also be a putative hormone receptor that transmits signal into nucleus (Calvo et al., 1999). 


\subsection{Genetic elements governing fruit body formation}

As there are many external determinants of fruit body formation, there are also some internal regulatory proteins that govern the spatial and temporal establishment of fruit body formation. These developmental regulators are encoded by a part of the genome, most of which encodes house keeping proteins that are necessary for normal vegetative development. Sexual development requires the spatial, temporal and the right dose of gene expression in order to yield proper-shaped and numbered cleistothecia. There are some number of genes whose presence is indispensible for fruit body establishment. Sexual development is typically controlled by genes that reside in the mating-type locus. The mating-type locus encodes proteins that have transcription factor properties and activates the transcription of pheromone and pheromone receptor encoding genes. Mutation in the mating type locus cause asexual organism (Coppin et al., 1997; Kronstad and Staben, 1997; Poggeler and Kuck, 2001).

Among the genes necessary for sexual fruit body formation the velvet factor comes first. VeA protein regulates the light-dependent sexual development of $A$. nidulans. In the darkness it is active and promotes sexual development while it is inactive or inactivated in the light (red light) and results in asexual conidiation (details in next part) (Mooney and Yager, 1990). Another factor involved in the number of fruit bodies is the fungal phytochrome protein of $A$. nidulans called FphA. Deletion of $f p h A$ stimulates cleistothecia production under red light which normally represses sexual development. However this increase in the number of cleistothecia production is not as much as in the darkness. This suggests that the red light phenomenon or red light inhibition of fruit body formation is a consequence of the phytochrome mediated signal transduction pathway (Blumenstein et al., 2005).

A UV mutagenesis screen in the early 1990s detected another set of genes chategorized as $n s d$ mutants (never in sexual development) that were defective in sexual development (Han et al., 1990; Han et al., 1994a; Han et al., 1994b). Among them there were $b s d$ mutants (blocked in sexual development) that were blocked at certain stages of sexual development and asd mutants (abbnormal in sexual development) that exhibited differences in morphology of sexual structures or timing of sexual development. Among the nsd mutants, $n s d D$ gene encodes a putative transcription factor which controls the fruit body formation and was characterised finally in 2001 (Han et al., 2001). nsdD is a GATA type of transcription factor encoding gene, deletion of which causes the loss of fruit body formation and overexpression of which promotes formation of sexual stage specific Hülle cell formation even under submersed liquid culture that usually respresses any kind of differentiation (Han et al., 2001). Another putative $\mathrm{Zn}(\mathrm{II})(2) \mathrm{Cys}(6)$ transcription factor encoding gene $\operatorname{ros} A$ 


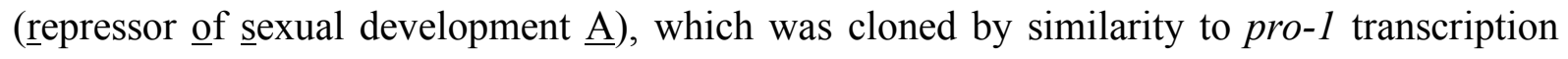
factor encoding gene of Sordaria macrospora, represses sexual development under inappropriate conditions such as low carbon source in liquid culture in contrast to pro- 1 of $S$. macrospora that has a positive role in sexual development of S. macrospora (Vienken et al., 2005). Deletion of $\operatorname{ros} A$ promotes the formation of Hülle cells in submerged culture but sexual development does not exceed beyond Hülle cell formation and never reaches to fruit body formation (Vienken et al., 2005). Another pro-1 related gene, nosA (number of sexual spores A) encodes a nuclear-localized putative $\mathrm{Zn}(\mathrm{II})(2) \mathrm{Cys}(6)$ transcription factor required for primordium maturation and is genetically connected to $\operatorname{ros} A$. Because $\operatorname{ros} A$ represses $\operatorname{nos} A$ expression it indicates that the balance of these two $\mathrm{Zn}(\mathrm{II})(2) \mathrm{Cys}(6)$ proteins determines the fate of vegetative hyphae to undergo sexual development (Vienken and Fischer, 2006). The $A$. nidulans steA encodes a homeodomain $\mathrm{C} 2 / \mathrm{H} 2-\mathrm{Zn}^{+2}$ finger transcription factor that is necessary for sexual production (Vallim et al., 2000). A steA $\Delta$ strain, despite being sterile, is able to form Hülle cells and cannot differentiate ascogenous tissue and fruiting bodies. STE12 of the budding yeast $S$. cerevisiae is the homolog of steA and encodes a transcription factor which is regulated by MAPK signal transduction and regulates cell identity, karyogamy, and morphogenesis (Mosch et al., 1996). In addition to these transcription factors, there are two more factors identified to be responsible both for asexual conidiation and sexual fruit body formation. The first is the medA gene that encodes a transctiption factor. Deletion of $m e d A$ in A. nidulans results in loss of celistothecia but the fungus is still able to form Hülle cells (Busby et al., 1996). The second is a basic helix-loop-helix (bHLH)-like transcription factor encoded by stuA gene of $A$. nidulans. Disruption of stuA brings about a completely acleistothecial phenotype and stuAD exhibits spatially abnormal conidiophores with spore production from the vesicles (Miller et al., 1991). stuA appears to be crucial for the appropriate spatial and medA for the correct temporal expression of the $\operatorname{brl} A$ gene which also encodes a Zn-coordinated transcription factor that is indispensable for conidiophore development during asexual sporulation. The absence of this locus removes conidiophores and the brlA mutant exhibit only bristle like hyphae (Adams et al., 1988).

In addition to these transcription factors, there are also different cell signaling pathways or physiological conditions controlling fruit body formation. Growth under amino acid starvation conditions permits the initiation of the sexual development program but prevent fruit body formation before meiosis. This block gives rise to microcleistothecia that are filled with ascogenous hypha instead of ascospores. The product of the yeast GCN4 homolog, $c p c A$ (cross-pathway control) of $A$. nidulans, which regulates the transcription of 
amino acid biosynthetic genes, encodes a leucine zipper putative transcription factor. Overexpression of $c p c A$ causes the same block in fruit body formation caused by amino acid starvation even in the absence of amino acid starvation suggesting that some intrinsic signals affect the sexual development of fruit bodies (Hoffmann et al., 2001). Although there are also some elements of signal transduction involved in the regulation of fruit body formation, the signal transduction compounds necessary for sexual development in A. nidulans are poorly understood. The reason behind this might be due to the fact that both asexual sporulation and sexual development processes and their regulatory signal transduction pathways are intermingled. Development of asexual conidiophores takes place earlier than sexual organs but some of the signals are also required for fruit body development. There are some phenotypes caused by mutations in $f l u$ ( $f l u f f y$ ) genes. $f l u$ mutations generate colonies with profuse aerial hyphae that look like the cotton wool (Wieser et al., 1994). Genetic analysis of $f l u$ mutations or their suppressors uncovered several signaling elements. They appear to be involved in the transmission of external signals into the nucleus. The prominent feature of $f l u$ mutations is that mutants are not capable of performing the sexual cycle.

Detailed inspection of the A. nidulans genome unveiled the nine genes (gprA-gprI) that have putative seven-transmembrane spanning G-PRCs (్-protein-coupled receptor). $\operatorname{gpr} A, \operatorname{gpr} B$ and $\operatorname{gprD}$ were shown to be crucial for the coordination of hyphal growth and sexual development (Han et al., 2004; Seo et al., 2004b). By similarity to $S$. cerevisiae Ste2p and Ste $3 p$ that encodes pheromone receptors, gprA and gprB were found to be homologous to Ste2p and Ste3p, respectively. Deletion of either gprA or gprB sparks the production of a few small cleistothecia that comprise of a reduced number of ascospores. A. nidulans gprA $\Delta / g$ prB $\Delta$ double knock-out is completely abolished in fruit body formation under homothallic conditions. In contrast, out-crossing of receptor mutants (gprA $/$ gprB $\Delta \mathrm{X}$ $\operatorname{gpr} A \Delta / \operatorname{gpr} B \Delta$ ) allows the fruit body and ascospore formation at wild type levels proposing that A. nidulans pheromone receptors GrpA and GprB are specifically required for selffertilization but not for complete sexual development (Seo et al., 2004b). Disruption of the gprD gene results in extremely restricted hyphal growth, delayed conidial germination and uncontrolled sexual development. It was suggested that $\operatorname{gprD}$ negatively regulates sexual development because elimination of sexual development reconstitutes both developmental and growth abnormalities (Han et al., 2004). Furthermore it was disclosed that growth defects caused by the loss of $g p r D$ is suppressed by either $\operatorname{gpr} A$ or $\operatorname{gpr} B$ deletions implying that GprD-mediated negative regulation of sexual development is hierarchically placed upstream 
of the two pheromone receptors, respectively GprA and GprB (Seo et al., 2004b). The environmental and internal factors that govern fruit body formation is depicted in Fig. 2.

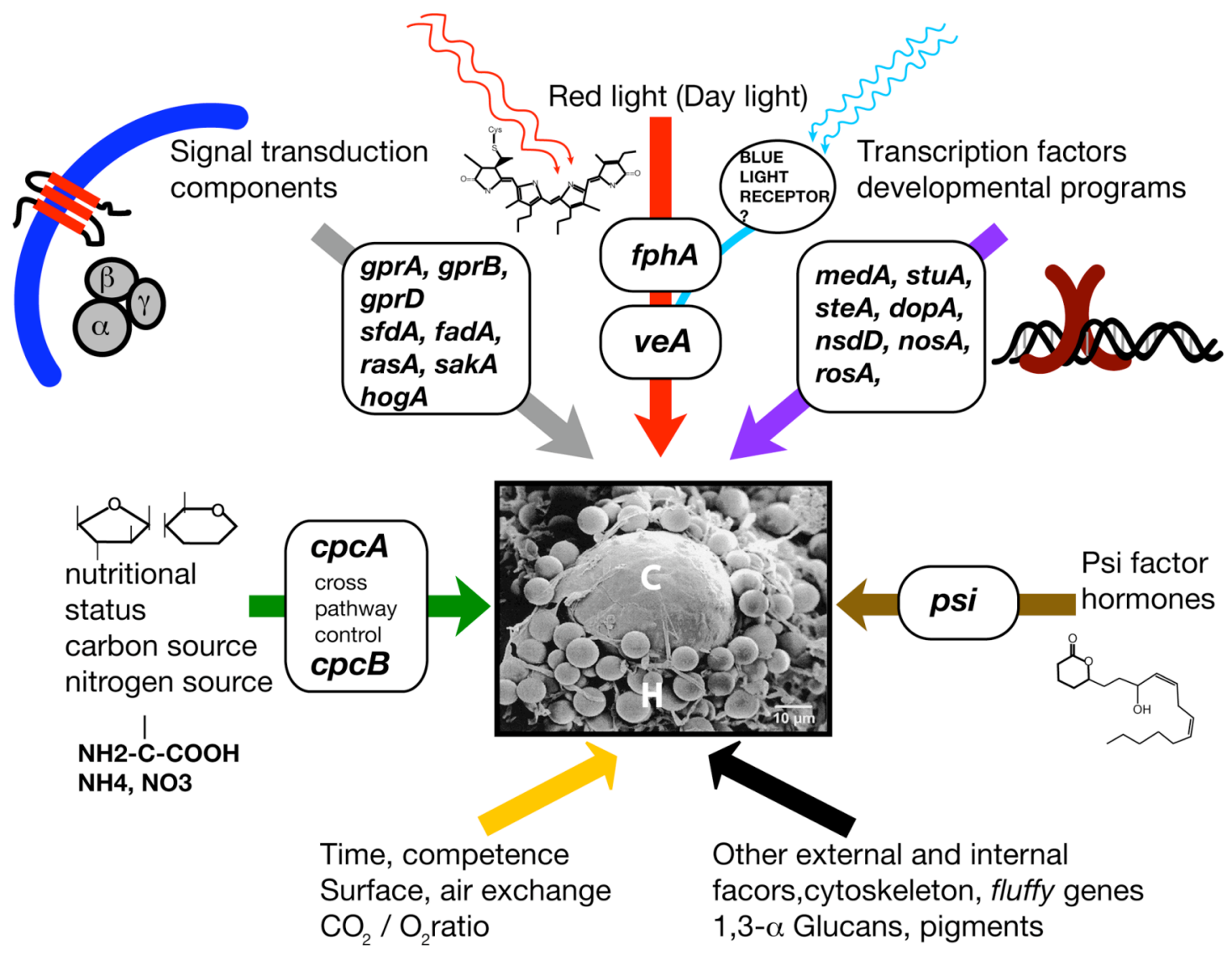

Figure 2: Factors that affect fruit body development in $\boldsymbol{A}$. nidulans. External (environmental) and internal (genetic) factors that are necessary in order to initiate and finalize a successful sexual development program. A scanning electron micrograph of a mature fruit body of $A$. nidulans is shown at the center. (C) is a mature cleistothecium filled with ascospores and $(\mathrm{H})$ Hülle cells that surround the cleistothecium (modified from Braus et al. 2002).

Some components of membrane receptors and heterotrimeric G-proteins have been determined to be involved in sexual development (Rosen et al., 1999). In A. nidulans, the Ga subunit, FadA, along with the G $\beta$ subunit, SfdA, was demonstrated to be an active player in signaling pathways that govern critical decisions in the ascocarp establishment. fadA mutant strains are unable to form cleistothecia. By contrast, a dominant-negative mutation in fadA $\left(f a d A^{\mathrm{G} 203 \mathrm{R}}\right)$ gene results in an increase in Hülle cell production but cannot give rise to cleistothecia. $s f d A \Delta$ also triggers the same kind of phenotype where no fruit bodies are formed (Rosen et al., 1999). Moreover flbA gene of $A$. nidulans, a homolog of yeast SST2, encodes an RGS protein (regulator of $\underline{\text { G-protein }}$ signaling) that functions as a GTPase activating protein, was shown to play a role in fruit body formation (Yu et al., 1996). Another group of players 
in the signaling pathways is ras proteins. Overexpression of constitutively inactive ras $A$ alleles results in an acleistothecial phenotype. Lowest ras protein activity is needed for sexual development but it is obvious that RAS- and RAS-like proteins function mostly in conidial germination and asexual development (Som and Kolaparthi, 1994; Osherov and May, 2000; Fillinger et al., 2002).

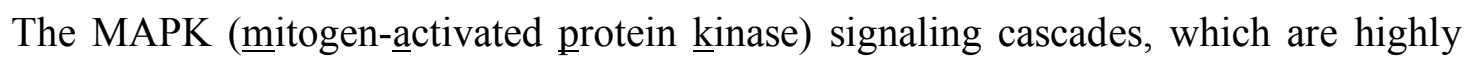
conserved pathways in the yeast systems $S$. cerevisiae and $S$. pombe, respond to various external stimuli by activating a variety of cellular programs such as mating and differentiation (Banuett, 1998). A crosstalk between the PKA and MAPK pathways in the mating, filamentous growth and stress response in the budding yeast has been shown (Mösch et al., 1996). It was demonstrated that the Hog1p yeast homolog SakA/HogA plays a role not only in stress signaling but also in sexual development. Deletion of $\operatorname{sakA}$ in $A$. nidulans results in premature fruit bodies with a temporal disruption of sexual development since the deletion strains generate cleisothecia 24 hours earlier than wild type strain (Kawasaki et al., 2002).

\subsection{Velvet (veA) gene of Aspergillus nidulans}

Among the group of gene products characterised so far to influence A. nidulans cleistothecia formation, the so-called velvet factor is the most ancient but also the most enigmatic one. In 1965, Käfer first described the phenotype of veAl mutants which form less aerial hyphae than a wild-type strain and which have a velvety appearance of older colonies on plates (Käfer, 1965). Moreover, veAl mutants show retarded sexual development and excessive asexual sporulation. It was demonstrated later that in a $v e A^{+}$background red light promotes the formation of conidiospores while veAl strains are not affected by this kind of illumination (Mooney et al., 1990; Mooney and Yager, 1990). Therefore, the veA gene product was proposed to be a negative regulator of asexual conidiation whose function could be suppressed by red light or day light (Champe et al., 1981; Mooney et al., 1990; Timberlake, 1990). It was shown that deletion of $A$. nidulans red light receptor $f p h A$ causes an increase in the number of Hülle cells under red light suggesting that there is a connection between velvet and phytochrome FphA (Blumenstein et al., 2005). The veA gene could be cloned and characterized demonstrating that the deletion of the veA gene results in an acleistothecial phenotype even under conditions that promote sexual development. Accordingly, overexpression results in Hülle cell formation even in submerged culture (Kim et al., 2002). This work also demonstrated that the veAl allele was formed as a result of $\mathrm{G}->$ $\mathrm{T}$ nucleotide substitution in the start codon of the wild-type $v e A$ gene (ATG $>$ ATT) leading 
to downstream translational initiation and synthesis of a truncated gene product. Moreover, velvet's role seems not to be restricted to sexual development in Aspergillus species. It was also shown that veA of $A$. parasticus affects sclerotial production as well as secondary metabolism and that a $v e A$ gene disruption in the asexual pathogen A. fumigatus reduces asexual sporulation depending on the nitrogen source (Calvo et al., 2004; Krappmann et al., 2005). It has been recently shown that the deletion of the heterothallic plant pathogen Fusarim verticilloides velvet gene has pleiotrophic effects on the development of the fungus regulating filamentous growth and the ratio of microconidia to macroconidia and cell wall formation ( $\mathrm{Li}$ et al., 2006).

Besides phenotypic analyses, no knowledge has been gained on the actual cellular function of VeA and its relationship to light dependency of the cleistothecia formation. As the gene product lacks similarity to characterised proteins outside the fungal kingdom, no further hints could be deduced from its amino acid sequence.

\section{Secondary metabolism}

Secondary metabolism is a typical feature of plant and fungal cells leading to the production of low-molecular-weight chemicals called secondary metabolites that have many potent physiological effects on living organisms. Digitalis, morphine and quinine belong to plant secondary metabolites whereas penicillin, cephalosporin, ergotate, aflatoxin, gliotoxin, sterigmatocystin (ST) are classified as fungal secondary metabolites (Keller et al., 2005). Primary metabolism of a cell is always required for survival and physiological activities whereas secondary metabolism is not crucial for survival under normal conditions and is only produced at a certain developmental stage and renders some benefits for the fungus as in the case of bacterial cleaning of ecological niches by penicillin products. After discovery of the anti-bacterial penicillin by Alexander Fleming, research on fungal secondary metabolites accelerated and many secondary metabolites were discovered. Fungal secondary metabolites have strong effects on the cell activities among which cytotoxic, mutagenic, carcinogenic, teratogenic, immunosuppressive, enzyme inhibitory effects are the most prominent ones. A recent survey of fungal metabolites disclosed that more than 1.500 fungal metabolites were found and characterized between 1993 and 2001 and more than half of these molecules had antifungal, antibacterial and antitumor activity (Keller et al., 2005).

Fungal secondary metabolites are classified into four groups depending on the chemical properties and the manner of synthesis. The main groups are polyketides, peptides, alkoloids and terpenes. Polyketides: They are the most abundant fungal secondary metabolites 
including the yellow $A$. nidulans spore pigment intermediate naphthopyrone (WA), the carcinogen aflatoxin (aftatoxin $\mathrm{B}_{1}, \mathrm{~B}_{2}, \mathrm{G}_{1}$ and $\mathrm{G}_{2}$ ) and the cholesterol-reducing compound lovastatin. Non-ribosomal peptides: penicillin G, cyclosporin and gliotoxin are among the important NRP (№n ribosomal peptides) group of chemicals. Terpenes: aristolochenes, caretenoids, gibberellins, trichothecenes that contain isoprene units. Indole alkoloids: derivatives of tryptophan and dimethylallyl pyrophosphate, gibberellin GA3, trichothecene T2 toxin and aristolochene (Keller et al., 2005).

The fungus Aspergillus nidulans, which has been utilized as a model system to analyse secondary metabolism in Aspergillus spp., produces the polyketide sterigmatocystin (ST) and aflatoxins (AF) which are related fungal secondary metabolites (Keller and Adams, 1995; Keller and Hohn, 1996). They are among the most toxic, mutagenic and carcinogenic natural products known (Trail et al., 1995; Sweeney and Dobson, 1999). The genes responsible for ST biosynthesis are placed in a cluster (Brown et al., 1996) which is shown in Fig. 3 . Structural genes involved in fungal secondary metabolism act as one locus (Keller et al., 2006). The regulation of the clustered genes is largely dependent on pathway specific transcription factors (Fernandes et al., 1998; Pedley and Walton, 2001), global regulators (Bok and Keller, 2004) and signal transduction pathways that connect the secondary metabolism with sporulation (Calvo et al., 2002). Transcription of the ST gene cluster is activated by a transcription factor called aflR that is located within the ST structural gene cluster (Fernandes et al., 1998). Further upstream regulation is conferred by LaeA, which is the master regulator of secondary metabolism in Aspergillus. spp. It is a methyl transferase possibly acting on histone methylation (Keller et al., 2005). Proposed mode of action of laeA on ST gene cluster regulation is given in Figure 3. Deletion of A. nidulans laeA causes the loss of expression of ST gene cluster including the transcription factor aflR (Bok and Keller, 2004), A. fumigatus laeAs strains have decreased virulence in a murine model (Bok et al., 2005). Fungal secondary metabolism is regulated by many factors and is associated with a specific stage of fungal development. It was shown that velvet gene of $A$. nidulans is involved in secondary metabolite regulation and deletion of veA gene results in loss of the ST metabolite which is in agreement with severe down-regulation of ST structural and aflR gene expression (Kato et al., 2003). It was also displayed that in the light, ST production is rather lower in comparison to darkness where much more ST is synthesized (Kato et al., 2003) suggesting that light has a negative effect on ST production, which is attributed to the velvet gene. In addition to ST production, expression of one of the penicillin biosynthesis genes, ipnA that encodes isopenicillin synthetase is repressed by veA. In contrast to ipnA, veA is 
necessary for the expression of $a c v A$, the key gene in the first step of penicillin biosynthesis encoding delta-(L-alpha-aminoadipyl)-L-cysteinyl-D-valine synthetase (Kato et al., 2003). However, there are controversial data for the role of veA on acvA (Sprote and Brakhage, 2007).
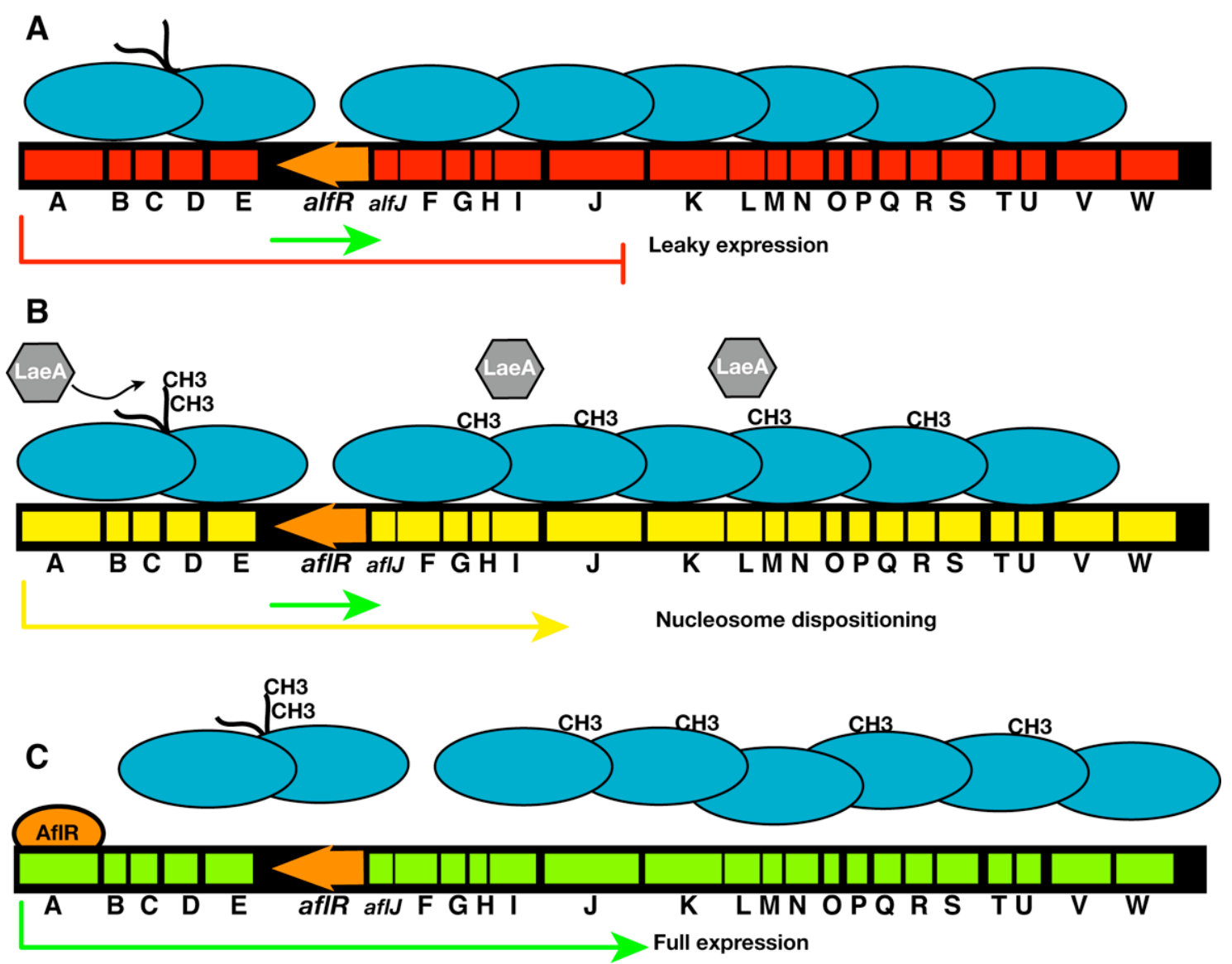

Figure 3: ST (Sterigmatocystin) biosynthesis gene cluster and regulation of gene expression in the ST cluster. Approximately 30 genes responsible for ST biosynthesis including the gene encoding the transcriptional regulatory protein aflR of the ST gene cluster are clustered and spans $65 \mathrm{~kb}$ in the subtelomeric region that is occupied by heterochromatin factors which represses gene expression. The cluster is transcirbed at the basal levels under normal conditions (A) Expression of the cluster increases upon activation by LaeA (global regulator of secondary metabolism) methyl transferase which probably acts on methylation of chromatin factors and results in an elevation of ST gene expression $(\mathbf{B}, \mathbf{C})$.

The exact mode of action of velvet in secondary metabolism is still elusive and only some genetic data support the concept of connection or coregulation. The idea of coregulation of secondary metabolism with development has been underestimated or overlooked for years. There have been proposals that some weak connections with development and secondary metabolism involving velvet (Kato et al., 2003) and some cell signaling components play a role (Calvo et al., 2002). To support these hypotheses, investigation at the 
level of protein is a prerequisite and any physical interaction between development and secondary metabolism regulators might explain the co-regulation phenomena.

\section{Aim of the work}

In this work, the blue light-dependent development of Aspergillus nidulans was investigated. Fungal development is a more complicated process that requires many environmental inputs and internal regulators. Blue light along with red light is one of the important environmental signals for the regulation of development. Phototropins are mainly responsible for blue light responses in fungi. However, there is another group protein family, cryptochromes/DNA photolyases, on which blue and UV light spectrum have regulatory effects. We were interested in the molecular mechanism of the blue light response in A. nidulans. The genome of $A$. nidulans contains only one cryptochrome/photolyase-like gene named cryA. However, many other fungi have more than one cryptochrome/photolyase-like protein encoding gene in their genome. As there are no fungal cryptochrome that have been characterized to date, in $A$. nidulans, cryptochrome/photolyase encoding gene cry $A$ had to be knocked out in order to gain further insight into the regulatory properties of the cryA gene product in the blue lightregulated fungal development. The main objective of the research was to investigate the role of cry $A$ in blue light perception and to try to find out if cryA had a genetical or direct connection with other developmental regulators. Another aim of this study was to analyse whether $\operatorname{cry} A$ was a real cryptochrome or a DNA repairing photolyase as the cryptochromes lack for DNA repair activity.

Genetical interaction between cryA and the other light-dependent player velvet prompted us to ask the question if there were any gene products that interact with VeA. Since veA of $A$. nidulans has various crucial roles for fungal development pathway, we wanted to investigate how velvet regulates light-dependent sexual development and other processes such as ST production. Therefore we aimed at targetting the interactome of velvet protein in order to find interaction partners or a cellular protein complex that relays the light signal to the nucleus. To that aim, the commonly used TAP tag (Tandem Affinity Purification) method, which was described by (Puig et al., 2001), had to be utilized (see chapter 4, page 110). In addition to elucidating the VeA interactome, we also analysed the Neurospora crassa velvetlike protein. We wondered whether the $N$. crassa velvet (ve-1) is conserved either structurally or functionally. Therefore, we wanted to investigate the function of ve-1 by knocking it out in a heterothallic organism and so as to reveal its role in the light-dependent development of $N$. 
crassa. The aim of the project was also to analyse the similarities and differences between $A$. nidulans veA and $N$. crassa ve-1. 


\section{References}

Adams, T.H., Boylan, M.T., and Timberlake, W.E. (1988). brlA is necessary and sufficient to direct conidiophore development in Aspergillus nidulans. Cell 54, 353-362.

Adams, T.H., Wieser, J.K., and Yu, J.H. (1998). Asexual sporulation in Aspergillus nidulans. Microbiol Mol Biol Rev 62, 35-54.

Agrios, G.N. (1997). Plant pathology. $4^{\text {th }}$ edition. (London, United Kingdom: Academic Press).

Ballario, P., Talora, C., Galli, D., Linden, H., and Macino, G. (1998). Roles in dimerization and blue light photoresponse of the PAS and LOV domains of Neurospora crassa white collar proteins. Mol Microbiol 29, 719-729.

Banuett, F. (1998). Signalling in the yeasts: an informational cascade with links to the filamentous fungi. Microbiol Mol Biol Rev 62, 249-274.

Bieszke, J.A., Braun, E.L., Bean, L.E., Kang, S., Natvig, D.O., and Borkovich, K.A. (1999). The nop-1 gene of Neurospora crassa encodes a seven transmembrane helix retinal-binding protein homologous to archaeal rhodopsins. Proc Natl Acad Sci U S A 96, 8034-8039.

Blumenstein, A., Vienken, K., Tasler, R., Purschwitz, J., Veith, D., Frankenberg-Dinkel, N., and Fischer, R. (2005). The Aspergillus nidulans phytochrome FphA represses sexual development in red light. Curr Biol 15, 1833-1838.

Bok, J.W., and Keller, N.P. (2004). LaeA, a regulator of secondary metabolism in Aspergillus spp. Eukaryot Cell 3, 527-535.

Bok, J.W., Balajee, S.A., Marr, K.A., Andes, D., Nielsen, K.F., Frisvad, J.C., and Keller, N.P. (2005). LaeA, a regulator of morphogenetic fungal virulence factors. Eukaryot Cell 4, 1574-1582. 
Borkovich, K.A., Alex, L.A., Yarden, O., Freitag, M., Turner, G.E., Read, N.D., Seiler, S., Bell-Pedersen, D., Paietta, J., Plesofsky, N., Plamann, M., GoodrichTanrikulu, M., Schulte, U., Mannhaupt, G., Nargang, F.E., Radford, A., Selitrennikoff, C., Galagan, J.E., Dunlap, J.C., Loros, J.J., Catcheside, D., Inoue, H., Aramayo, R., Polymenis, M., Selker, E.U., Sachs, M.S., Marzluf, G.A., Paulsen, I., Davis, R., Ebbole, D.J., Zelter, A., Kalkman, E.R., O'Rourke, R., Bowring, F., Yeadon, J., Ishii, C., Suzuki, K., Sakai, W., and Pratt, R. (2004). Lessons from the genome sequence of Neurospora crassa: tracing the path from genomic blueprint to multicellular organism. Microbiol Mol Biol Rev 68, 1-108.

Braus, G.H., Krappmann, S., and Eckert, S. (2002). Sexual development in ascomycetes: Fruit body formation in Aspergillus nidulans. In Molecular Biology of Fungal Development, Osiewacz, ed (New York: Marcel Dekker Inc), pp. 215-244.

Brown, D.W., Yu, J.H., Kelkar, H.S., Fernandes, M., Nesbitt, T.C., Keller, N.P., Adams, T.H., and Leonard, T.J. (1996). Twenty-five coregulated transcripts define a sterigmatocystin gene cluster in Aspergillus nidulans. Proc Natl Acad Sci U S A 93, 1418-1422.

Busby, T.M., Miller, K.Y., and Miller, B.L. (1996). Suppression and enhancement of the Aspergillus nidulans medusa mutation by altered dosage of the bristle and stunted genes. Genetics 143, 155-163.

Calvo, A.M., Hinze, L.L., Gardner, H.W., and Keller, N.P. (1999). Sporogenic effect of polyunsaturated fatty acids on development of Aspergillus spp. Appl Environ Microbiol 65, 3668-3673.

Calvo, A.M., Wilson, R.A., Bok, J.W., and Keller, N.P. (2002). Relationship between secondary metabolism and fungal development. Microbiol Mol Biol Rev 66, 447-459, table of contents.

Calvo, A.M., Bok, J., Brooks, W., and Keller, N.P. (2004). veA is required for toxin and sclerotial production in Aspergillus parasiticus. Appl Environ Microbiol 70, 47334739 . 
Casas-Flores, S., Rios-Momberg, M., Bibbins, M., Ponce-Noyola, P., and HerreraEstrella, A. (2004). BLR-1 and BLR-2, key regulatory elements of photoconidiation and mycelial growth in Trichoderma atroviride. Microbiology 150, 3561-3569.

Champe, S.P., and el-Zayat, A.A. (1989). Isolation of a sexual sporulation hormone from Aspergillus nidulans. J Bacteriol 171, 3982-3988.

Champe, S.P., Rao, P., and Chang, A. (1987). An endogenous inducer of sexual development in Aspergillus nidulans. J Gen Microbiol 133, 1383-1387.

Champe, S.P., Kurtz, M.B., Yager, L.N., Butnick, N.J., and Axelrod, D.E. (1981). Spore formation in Aspergillus nidulans: competence and other developmental processes. In Fungal spores: Morphogenic Controls, H.R. Hohl and G. Turian, eds (New York: Academic Press), pp. 255-276.

Cheng, P., Yang, Y., Gardner, K.H., and Liu, Y. (2002). PAS domain-mediated WC1/WC-2 interaction is essential for maintaining the steady-state level of WC-1 and the function of both proteins in circadian clock and light responses of Neurospora. Mol Cell Biol 22, 517-524.

Coppin, E., Debuchy, R., Arnaise, S., and Picard, M. (1997). Mating types and sexual development in filamentous ascomycetes. Microbiol Mol Biol Rev 61, 411-428.

Degli-Innocenti, F., Chambers, J.A., and Russo, V.E. (1984). Conidia induce the formation of protoperithecia in Neurospora crassa: further characterization of white collar mutants. J Bacteriol 159, 808-810.

Elvin, M., Loros, J.J., Dunlap, J.C., and Heintzen, C. (2005). The PAS/LOV protein VIVID supports a rapidly dampened daytime oscillator that facilitates entrainment of the Neurospora circadian clock. Genes Dev 19, 2593-2605.

Fankhauser, C., and Staiger, D. (2002). Photoreceptors in Arabidopsis thaliana: light perception, signal transduction and entrainment of the endogenous clock. Planta 216, $1-16$. 
Fernandes, M., Keller, N.P., and Adams, T.H. (1998). Sequence-specific binding by Aspergillus nidulans AflR, a C6 zinc cluster protein regulating mycotoxin biosynthesis. Mol Microbiol 28, 1355-1365.

Fillinger, S., Chaveroche, M.K., Shimizu, K., Keller, N., and d'Enfert, C. (2002). cAMP and ras signalling independently control spore germination in the filamentous fungus Aspergillus nidulans. Mol Microbiol 44, 1001-1016.

Froehlich, A.C., Noh, B., Vierstra, R.D., Loros, J., and Dunlap, J.C. (2005). Genetic and molecular analysis of phytochromes from the filamentous fungus Neurospora crassa. Eukaryot Cell 4, 2140-2152.

Han, D.M., Han, Y.J., Chae, K.S., Jahng, K.Y., and Lee, Y.H. (1994a). Effects of various carbon sources on the development of Aspergillus nidulans with velA+ or veAl allele. Korean Journal Mycol 22, 332-337.

Han, D.M., Han, Y.J., Lee, Y.H., Jahng, K.Y., Jahng, S.H., and Chae, K.S. (1990). Inhibitory conditions of asexual development and their application for the screening of mutants defective in sexual development. Korean Journal Mycol 18, 225-232.

Han, D.M., Han, Y.J., Kim, J.H., Jahng, K.Y., Chung, Y.S., Chung, J.H., and Chae, K.S. (1994b). Isolation and characterization of $n s d$ mutants in Aspergillus nidulans. Korean Journal Mycol 22, 1-7.

Han, K.H., Seo, J.A., and Yu, J.H. (2004). A putative G protein-coupled receptor negatively controls sexual development in Aspergillus nidulans. Mol Microbiol 51, 1333-1345.

Han, K.H., Han, K.Y., Yu, J.H., Chae, K.S., Jahng, K.Y., and Han, D.M. (2001). The $n s d D$ gene encodes a putative GATA-type transcription factor necessary for sexual development of Aspergillus nidulans. Mol Microbiol 41, 299-309.

Harding, R.W., and Melles, S. (1983). Genetic Analysis of Phototropism of Neurospora crassa Perithecial Beaks Using White Collar and Albino Mutants. Plant Physiol 72, 996-1000. 
He, Q., and Liu, Y. (2005). Molecular mechanism of light responses in Neurospora: from light-induced transcription to photoadaptation. Genes Dev 19, 2888-2899.

Hoffmann, B., Valerius, O., Andermann, M., and Braus, G.H. (2001). Transcriptional autoregulation and inhibition of mRNA translation of amino acid regulator gene $c p c A$ of filamentous fungus Aspergillus nidulans. Mol Biol Cell 12, 2846-2857.

Idnurm, A., and Heitman, J. (2005). Light controls growth and development via a conserved pathway in the fungal kingdom. PLoS Biol 3, e95.

Idnurm, A., Rodriguez-Romero, J., Corrochano, L.M., Sanz, C., Iturriaga, E.A., Eslava, A.P., and Heitman, J. (2006). The Phycomyces madA gene encodes a blue-light photoreceptor for phototropism and other light responses. Proc Natl Acad Sci U S A 103, 4546-4551.

Käfer, E. (1965). Origins of translocations in Aspergillus nidulans. Genetics 52, 217-232.

Kakiuchi, Y., Takahashi, T., Murakami, A., and Ueda, T. (2001). Light irradiation induces fragmentation of the plasmodium, a novel photomorphogenesis in the true slime mold Physarum polycephalum: action spectra and evidence for involvement of the phytochrome. Photochem Photobiol 73, 324-329.

Kato, N., Brooks, W., and Calvo, A.M. (2003). The expression of sterigmatocystin and penicillin genes in Aspergillus nidulans is controlled by veA, a gene required for sexual development. Eukaryot Cell 2, 1178-1186.

Kawasaki, L., Sanchez, O., Shiozaki, K., and Aguirre, J. (2002). SakA MAP kinase is involved in stress signal transduction, sexual development and spore viability in Aspergillus nidulans. Mol Microbiol 45, 1153-1163.

Keller, N., Bok, J., Chung, D., Perrin, R.M., and Keats Shwab, E. (2006). LaeA, a global regulator of Aspergillus toxins. Med Mycol 44 Suppl, 83-85. 
Keller, N.P., and Adams, T.H. (1995). Analysis of a mycotoxin gene cluster in Aspergillus nidulans. SAAS Bull Biochem Biotechnol 8, 14-21.

Keller, N.P., and Hohn, T.M. (1996). Metabolic pathway gene clusters in filamentous fungi. Fungal Genet Biol 21, 17-29.

Keller, N.P., Turner, G., and Bennett, J.W. (2005). Fungal secondary metabolism - from biochemistry to genomics. Nat Rev Microbiol 3, 937-947.

Kim, H., Han, K., Kim, K., Han, D., Jahng, K., and Chae, K. (2002). The veA gene activates sexual development in Aspergillus nidulans. Fungal Genet Biol 37, 72-80.

Krappmann, S., Bayram, O., and Braus, G.H. (2005). Deletion and allelic exchange of the Aspergillus fumigatus veA locus via a novel recyclable marker module. Eukaryot Cell 4, 1298-1307.

Kronstad, J.W., and Staben, C. (1997). Mating type in filamentous fungi. Annu Rev Genet 31, 245-276.

Kues, U., and Liu, Y. (2000). Fruiting body production in Basidiomycetes. Appl Microbiol Biotechnol 54, 141-152.

Latge, J.P. (1999). Aspergillus fumigatus and aspergillosis. Clin Microbiol Rev 12, 310-350.

Li, S., Myung, K., Guse, D., Donkin, B., Proctor, R.H., Grayburn, W.S., and Calvo, A.M. (2006). FvVE1 regulates filamentous growth, the ratio of microconidia to macroconidia and cell wall formation in Fusarium verticillioides. Mol Microbiol 62, 1418-1432.

Liu, Y., He, Q., and Cheng, P. (2003). Photoreception in Neurospora: a tale of two White Collar proteins. Cell Mol Life Sci 60, 2131-2138. 
Miller, K.Y., Toennis, T.M., Adams, T.H., and Miller, B.L. (1991). Isolation and transcriptional characterization of a morphological modifier: the Aspergillus nidulans stunted (stuA) gene. Mol Gen Genet 227, 285-292.

Montgomery, B.L. (2007). Sensing the light: photoreceptive systems and signal transduction in cyanobacteria. Mol Microbiol 64, 16-27.

Mooney, J.L., and Yager, L.N. (1990). Light is required for conidiation in Aspergillus nidulans. Genes Dev 4, 1473-1482.

Mooney, J.L., Hassett, D.E., and Yager, L.N. (1990). Genetic analysis of suppressors of the veA1 mutation in Aspergillus nidulans. Genetics 126, 869-874.

Mösch, H.U., Roberts, R.L., and Fink, G.R. (1996). Ras2 signals via the Cdc42/Ste20/mitogen-activated protein kinase module to induce filamentous growth in Saccharomyces cerevisiae. Proc Natl Acad Sci U S A 93, 5352-5356.

Osherov, N., and May, G. (2000). Conidial germination in Aspergillus nidulans requires RAS signaling and protein synthesis. Genetics 155, 647-656.

Pedley, K.F., and Walton, J.D. (2001). Regulation of cyclic peptide biosynthesis in a plant pathogenic fungus by a novel transcription factor. Proc Natl Acad Sci U S A 98, 14174-14179.

Poggeler, S., and Kuck, U. (2001). Identification of transcriptionally expressed pheromone receptor genes in filamentous ascomycetes. Gene 280, 9-17.

Puig, O., Caspary, F., Rigaut, G., Rutz, B., Bouveret, E., Bragado-Nilsson, E., Wilm, M., and Seraphin, B. (2001). The tandem affinity purification (TAP) method: a general procedure of protein complex purification. Methods 24, 218-229.

Purschwitz, J., Muller, S., Kastner, C., and Fischer, R. (2006). Seeing the rainbow: light sensing in fungi. Curr Opin Microbiol 9, 566-571. 
Rockwell, N.C., Su, Y.S., and Lagarias, J.C. (2006). Phytochrome structure and signaling mechanisms. Annu Rev Plant Biol 57, 837-858.

Rosen, S., Yu, J.H., and Adams, T.H. (1999). The Aspergillus nidulans sfaD gene encodes a $\mathrm{G}$ protein beta subunit that is required for normal growth and repression of sporulation. Embo J 18, 5592-5600.

Sancar, A. (2000). Cryptochrome: the second photoactive pigment in the eye and its role in circadian photoreception. Annu Rev Biochem 69, 31-67.

Schwerdtfeger, C., and Linden, H. (2000). Localization and light-dependent phosphorylation of white collar 1 and 2, the two central components of blue light signaling in Neurospora crassa. Eur J Biochem 267, 414-422.

Seo, J.A., Han, K.H., and Yu, J.H. (2004). The gprA and gprB genes encode putative G protein-coupled receptors required for self-fertilization in Aspergillus nidulans. Mol Microbiol 53, 1611-1623.

Serlupi-Crescenzi, O., Kurtz, M.B., and Champe, S.P. (1983). Developmental defects resulting from arginine auxotrophy in Aspergillus nidulans. J Gen Microbiol 129, 3535-3544.

Smith, S.E., and Read, D.J. (1997). Mycorrhizal symbiosis. (New York, N.Y.: Academic Press, Inc).

Sohn, K.T., and Yoon, K.S. (2002). Ultrastructural study on the cleistothecium development in Aspergillus nidulans. Mycobiology 30, 117-127.

Som, T., and Kolaparthi, V.S. (1994). Developmental decisions in Aspergillus nidulans are modulated by Ras activity. Mol Cell Biol 14, 5333-5348.

Springer, M.L., and Yanofsky, C. (1989). A morphological and genetic analysis of conidiophore development in Neurospora crassa. Genes Dev 3, 559-571. 
Sprote, P., and Brakhage, A.A. (2007). The light-dependent regulator velvet $A$ of Aspergillus nidulans acts as a repressor of the penicillin biosynthesis. Arch Microbiol.

Starostzik, C., and Marwan, W. (1995). A photoreceptor with characteristics of phytochrome triggers sporulation in the true slime mould Physarum polycephalum. FEBS Lett 370, 146-148.

Sweeney, M.J., and Dobson, A.D. (1999). Molecular biology of mycotoxin biosynthesis. FEMS Microbiol Lett 175, 149-163.

Talora, C., Franchi, L., Linden, H., Ballario, P., and Macino, G. (1999). Role of a white collar-1-white collar-2 complex in blue-light signal transduction. Embo J 18, 49614968.

Timberlake, W.E. (1990). Molecular genetics of Aspergillus development. Annu Rev Genet 24, 5-36.

Trail, F., Mahanti, N., and Linz, J. (1995). Molecular biology of aflatoxin biosynthesis. Microbiology 141 ( Pt 4), 755-765.

Vallim, M.A., Miller, K.Y., and Miller, B.L. (2000). Aspergillus SteA (sterile12-like) is a homeodomain-C2/H2-Zn+2 finger transcription factor required for sexual reproduction. Mol Microbiol 36, 290-301.

Vienken, K., and Fischer, R. (2006). The Zn(II)2Cys6 putative transcription factor NosA controls fruiting body formation in Aspergillus nidulans. Mol Microbiol 61, 544-554.

Vienken, K., Scherer, M., and Fischer, R. (2005). The Zn(II)2Cys6 putative Aspergillus nidulans transcription factor repressor of sexual development inhibits sexual development under low-carbon conditions and in submersed culture. Genetics 169, 619-630.

Wainright, M. (1992). An introduction to fungal biotechnology. (New York: John Wiley \& Sons, Inc.). 
Wieser, J., Lee, B.N., Fondon, J., 3rd, and Adams, T.H. (1994). Genetic requirements for initiating asexual development in Aspergillus nidulans. Curr Genet 27, 62-69.

Yu, J.H., Wieser, J., and Adams, T.H. (1996). The Aspergillus FlbA RGS domain protein antagonizes $\mathrm{G}$ protein signaling to block proliferation and allow development. Embo J 15, 5184-5190. 


\title{
Chapter 2
}

\section{More than a repair enzyme: Aspergillus nidulans photolyase-like CryA is a regulator of sexual development}

\begin{abstract}
Cryptochromes are blue light receptors that are believed to have evolved from the DNA photolyase protein family. The genomes of many organisms encode genes for both DNA photolyases as well as cryptochromes. However, in the genome of the filamentous fungus Aspergillus nidulans, only one cryptochrome/photolyase-encoding gene termed cryA was identified. N- and C- terminal sGFP fusions of CryA constitutively localise to the nucleus. Deletion of the cryA gene triggers sexual differentiation under inappropriate culture conditions and results in upregulation of transcripts encoding regulators of fruiting body formation. Strikingly, CryA exhibits photorepair activity as demonstrated by heterologous complementation of a DNA-repair deficient E. coli recipient; this is also substantiated by overexpression in an $A$. nidulans $u v s B \Delta$ genetic background while the single deletion $\operatorname{cry} A \Delta$ strain does not show increased sensitivity towards UV-induced damage. CryA act as a UVA-blue light receptor repressing cleistothecia formation under 350-370nm light. We propose that the cryptochrome/photolyase-like protein CryA represents a novel type of CPD I photolyase that bears a regulatory function as well as DNA repair activity and therefore might serve as a paradigm for an evolutionary transition state between photolyases and cryptochromes. Additionally, these data suggest that gene regulatory functions of class I CPD photolyases may have to be reconsidered.
\end{abstract}




\section{Introduction}

Cryptochromes (CRYs) are blue-light receptors and share great sequence homologies with DNA-photolyases. Members of the CRY/photolyase protein family are widely distributed among the three domains of life. They are presently divided into five subfamilies according to phylogenetic relationships and functions: class I cyclobutane pyrimidine dimer (CPD) photolyases, class II CPD photolyases, plant cryptochromes, animal cryptochromes including (6-4) photolyases, and the so-called CRY-DASH proteins. (reviewed by (Lin and Todo, 2005)). Even though members of the subfamilies are very similar in terms of sequence and structure, their functions are quite divergent. CRYs are usually nuclear localized to control gene expression (Lin and Todo, 2005). In animals, they play a role in modulating daily physiological and behavioural rhythms by mediating the entrainment of the circadian clock in response to day light (Cashmore, 2003; Lin and Todo, 2005) whereas in plants they are also involved in regulating photomorphogenetic responses such as stimulation of leaf expansion and photoperiodic flowering (Lin and Shalitin, 2003). The third subfamily of the CRY group called CRY-DASH has been identified in several organisms. The cyanobacteria (Synechocystis), Xenopus laevis, Danio rerio, Arabidopsis thaliana and the filamentous ascomycete Neurospora crassa are among them (Daiyasu et al., 2004; Lin and Todo, 2005). The exact function for members of the CRY-DASH family was elusive but recent data imply that they are actually photolyases that specifically repair CPDs in single stranded DNA (Selby and Sancar, 2006).

Class I and class II CPD photolyases share only limited sequence homology. While class I CPD photolyases are mainly found in prokaryotes and fungi, class II members are distributed over a broader range of organisms but show lesser sequence homologies to CRYs (Yasui et al., 1994; Kanai et al., 1997). CPD photolyases repair UV-induced DNA damage by employing a mechanism referred to as photorepair: they can absorb light in the blue spectrum to transfer an excited electron from a FAD cofactor to an enzyme-bound CPD and thereby cleave it (Sancar, 1990; Sancar, 1994).

Cryptochromes which also bind FAD as a cofactor and absorb wavelength in the bluelight spectrum are currently considered to exhibit no photorepair activity even though there has been a weak photorepair activity reported for members of the cry-DASH subfamily (Daiyasu et al., 2004). The structure of CRYs is characterized by an N-terminal photolyaserelated (PHR) region and a C-terminal domain varying in length. Members of the cry-DASH subfamily are missing for this C-terminal domain completely (Daiyasu et al., 2004). The PHR domain is the most conserved region of this group of proteins and contains similar cofactors 
as the photolyases. These cofactors play a crucial role in electron transfer reactions. There are, however, different findings concerning the functional parts of CRY proteins: In Xenopus and Drosophila the PHR-domain is physiologically active in the absence of the C-terminal domain. On the other hand, the C-terminal domain is important for localization or protein stability and its expression in Arabidopsis even results in a constitutive growth response (Lin and Todo, 2005). For the Arabidopsis thaliana CRYs, it was demonstrated that they are phosphorylated in response to light and several mechanisms of gene expression control have been proposed, which range from interactions with proteins directly or indirectly involved in the regulation of gene expression through to direct chromatin interactions (Lin and Todo, 2005). In mice, CRYs have been found to also affect the activity, interaction, degradation or nuclear trafficking of clock components in light-dependent and -independent manners (Griffin et al., 1999; Lin and Todo, 2005)

Most information gathered on light-mediated regulation in fungi deals with phototropin blue-light receptors. The white collar phototropin $\mathrm{WC}-1$ of the ascomycete $N$. crassa regulates fungal metabolism, development, mating, spore formation, and the circadian clock in response to blue- or UV-light (Liu et al., 2003). Zygomycetes and basidiomycetes also display conserved blue-light responses (Idnurm and Heitman, 2005; Idnurm et al., 2006). A. nidulans can undergo asexual and sexual reproduction depending on different environmental factors such as light or oxygen limitation. The asexual life-cycle of A. nidulans is characterized by the appearance of conidiophores that form asexual spores (conidia) by repeated mitotic divison from stem cell-like structures (reviewed in Adams et al., 1998). Alternatively, in the sexual reproductive cycle of $A$. nidulans, mycelia aggregate and supportive Hülle cells accompanied with ascospore-bearing fruiting bodies (cleistothecia) are formed (Braus et al., 2002a). In a wild-type strain, fruit body formation is repressed by light, aeration and growth in submerged culture while it is promoted by the presence of a medium/air interface and the absence of light. Until now, only a response to red-light illumination, for which the fungal phytochrome FphA was proposed to be one of the photosensors, has been characterised in detail in A. nidulans (Blumenstein et al., 2005).

Among the several genes that have been characterised as regulators of fruit body formation and the deletion of which results in complete loss of cleistothecia formation, the gene product of velvet $A$ (veA) exhibits a prominent role in mediating specifically lightdependent sexual development (Kim et al., 2002). Putative transcription factors are promising key regulators of differentiation and a variety of such has been characterised to date with respect to sexual development of $A$. nidulans: for instance, deletion of the so-called $n s d D$ 
(never in sexual development) locus encoding a GATA-type transcription factor results in a completely asexual phenotype (Han et al., 2001). Recently, the zinc finger transcription factor NosA was described to affect the number of ascospores (Vienken and Fischer, 2006). Besides the positive regulators of fruit body formation also factors that play a negative role have been

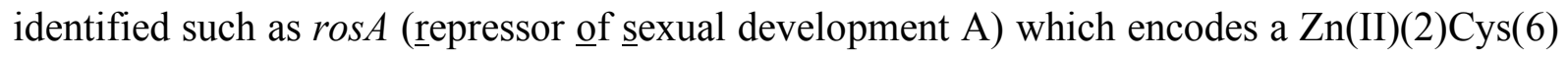
transcription factor. The deletion of $\operatorname{ros} A$ results in the formation of Hülle cells even in submerged culture (Vienken et al., 2005). In general, interactions of known regulators of the sexual reproductive pathway with sensors of environmental conditions or components of signal transduction cascades are still poorly understood.

Recently, the genome of the homothallic ascomycete $A$. nidulans has been published (Galagan et al., 2005b). Inspection of the sequence indicates one putative CRY/photolyaselike gene here termed cryA with the capacity to encode a protein of 567 aa that has a PHR domain and a small C-terminal extension. We made efforts to elucidate the cellular role of this CRY/photolyase-like gene during the A. nidulans life-cycle and data obtained from deletion, localization, and expression studies suggest that CryA holds a dual function as it appears to regulate gene expression in A. nidulans both in a light-dependent and independent manner and exhibits DNA photorepair activity in a heterologous E.coli test system. To our knowledge, this is the first instance where a member of a cryptochrome/photolyase-like protein family exhibits both regulatory and repair activity. This finding might give the first hints as to how cryptochromes have evolved from DNA photolyases. 


\section{Materials and Methods}

Sequence analyses. Sequence of CryA from A. nidulans was retrieved from the NATIONAL CENTER FOR BioteChNOLOGy INFORMATION (NCBI, gi: 40747330). Reference sequences were selected according to Daiyasu et al. (Daiyasu et al., 2004) and were also retrieved from the NCBI Entrez Protein database (see legend to figure 4C for corresponding gene identifier numbers). Multiple sequence alignments were carried out using T-COFFEE, ClustalW, and MAFFT with default parameters (Thompson et al., 1994; Notredame et al., 2000; Katoh et al., 2005). Phylogenetic trees were estimated by maximum parsimony (PAUP), neighbor-joining (PAUP) and maximum-likelihood (PhyML) methods using standard parameters (Guindon and Gascuel, 2003; Swofford, 2003); Bootstrapping was performed with 500 repetitions. Consensus trees were constructed using Clann (Creevey and McInerney, 2005) and visualized with TReeillustrator (Trooskens et al., 2005). Following orthonormal encoding of the alignments, principal component analysis, k-nearest-neighbour rule and nearest mean classification were computed essentially as described in (Duda et al., 2001).

Strains, media, and growth conditions. Fungal strains used during this study are listed in Table 1. The FGSC A4 strain served as wild-type recipient for deletion of cryA and strains AGB152 and AAH14 (uvsBA) were used for overexpression experiments (Hofmann and Harris, 2000; Busch et al., 2003). Standard laboratory Escherichia coli strains DH5 $\alpha$ and MACH-1 (INVITROGEN) were utilized for preparation of plasmid DNA and were propagated in $\mathrm{LB}$ medium ( $1 \%$ tryptone, $0.5 \%$ yeast extract, $1 \% \mathrm{NaCl}$ ) supplemented with 100-150 $\mu \mathrm{g} \cdot \mathrm{ml}^{-1}$ ampicillin, $40 \mu \mathrm{g} \cdot \mathrm{ml}^{-1}$ kanamycin, $2040 \mu \mathrm{g} \cdot \mathrm{ml}^{-1}$ tetracycline. Minimal medium $(0.52$ $\mathrm{g} \cdot 1^{-1} \mathrm{KCl}, 0.52 \mathrm{~g} \cdot \mathrm{l}^{-1} \mathrm{MgSO}_{4}, 1.52 \mathrm{~g} \cdot \mathrm{l}^{-1} \mathrm{KH}_{2} \mathrm{PO}_{4}, 0.1 \%$ trace element solution, $\mathrm{pH}$ 6.5) supplemented with appropriate amounts of 4-aminobenzoic acid (PABA, $1 \mu \mathrm{g} \cdot \mathrm{ml}^{-1}$ ), Uracil $\left(50 \mu \mathrm{g} \cdot \mathrm{ml}^{-1}\right)$, Pyridoxine $\left(0.05 \mu \mathrm{g} \cdot \mathrm{ml}^{-1}\right)$, nourseothricin-dihydrogen sulfate (clonNAT from WERnER BIOAGENTS, 100-120 $\mu \mathrm{g} \cdot \mathrm{ml}^{-1}$ ), pyrithiamine (TAKARA Bio Inc, $0.1 \mu \mathrm{g} \cdot \mathrm{ml}^{-1}$ ) was used for propagation of the fungal strains. $1 \% D$-glucose was used as carbon source together with $10 \mathrm{mM}$ ammonium or nitrate as source of nitrogen.

Transformation procedures. E. coli cells were transformed after calcium/manganesetreatment (Hanahan et al., 1991); A. nidulans was transformed by polyethylene glycolmediated fusion of protoplasts as described in detail by (Punt and van den Hondel, 1992). 
Recombinant DNA procedures and hybridisation techniques. Standard protocols were followed to generate recombinant DNA constructs (Sambrook et al., 1989a). Mostly, Taq, Pfu (MBI FERMENTAS), and Phusion ${ }^{\mathrm{TM}}$ (NEW ENGLAND BIOLABS) were used in polymerase chain reactions (Saiki et al., 1986), and crucial cloning steps were confirmed by sequence determination. Fungal genomic DNAs were prepared from ground mycelia (Kolar et al., 1988), and Southern blot analyses were performed as described (Southern, 1975). Total RNA samples were purified using the TRIzol reagent (INVITROGEN) followed by Northern hybridization according to the protocols cited by (Brown and Mackey, 1997). The StRATAgene PRIME-IT II kit was used to radioactively label hybridisation probes in the presence of $\left[\alpha-{ }^{32} \mathrm{P}\right] \mathrm{dATP}$ (Feinberg and Vogelstein, 1983). To produce autoradiographs, washed membranes were exposed to KoDAK X-OMAT films. Sequence data were analysed using the LASERGENE software package from DNASTAR (Thompson et al., 1994). cDNAs for RT-PCR were synthesized with the REVERTAID ${ }^{\text {TM }}$ First Strand cDNA Synthesis kit (MBI FERMENTAS) according to the manufacturer's protocol using equal amounts of DNA-free RNAs as input that had been isolated from different developmental stages.

Plasmid constructions. Plasmids constructed and used throughout this study are listed in Table 2. Oligonucleotide sequences are given in Table 3. Construction of the cryA replacement cassette in pME3181 was carried out as follows: the $1.8 \mathrm{~kb}$ cry A 3 ' flanking region was amplified $(\mathrm{OZG} 9 / 10)$ and inserted into the EcoRV site of pBluescript KS II (STRATAGENE) then the $1.8 \mathrm{~kb}$ cryA 5 'flanking sequence was amplified (OZG5/6) and cloned in the AatII site of 3'cryA DS-bearing plasmid, and finally a ptrA pyrithiamine resistance cassette with compatible $S f i$ I ends was inserted into the $S f i$ sites of the resulting plasmid. A $5.6 \mathrm{~kb}$ cryAA cassette was released from pME3181 by $B g l \mathrm{II} /$ NotI digestion prior to $A$. nidulans transformation to yield the cryA $\Delta$ deletion strain AGB288. A $6.8 \mathrm{~kb}$ cryA genomic fragment was sub-cloned into pGEM5 (SpeI/NsiI) creating pME3182. For construction of the complementation cassette using primers (OZG189/192) nat ${ }^{R}$ cassette was amplified from pNV1 and 3 UTR of cryA was amplified from the genomic (OZG191/10) DNA and these two fragments were fused by fusion PCR. The resulting $n a t^{R}+3$ UTR fusion fragment was cloned in pCR-Blunt II-TOPO (INVITROGEN). VspI site of this plasmid served as recipient for OZG199/200 amplified and $V s p$ I digested 5' UTR + cryA ORF from pME3182. This insertion resulted in complementation plasmid pME3183 from which $6.5 \mathrm{~kb}$ complementation fragment was released by PfIMI digest and used for complementation of cryA $\Delta$ mutant. For localization experiments, $\mathrm{N}$ - and C-sgfp-cryA fusions were constructed by one step fusion PCR: cryA 
cDNA was amplified from a cDNA library (OZG7/8 for N- and OZG7/89-noStop for Cterminal fusions, respectively) and sgfp was amplified with OZG 29/87 and OZG1/88, accordingly. $s g f p$ and cryA amplicons served as templates in PCRs to produce $s g f p:: c r y A$ and cryA::sgfp fusions that were cloned into the PmeI site of pME3160 yielding pME3184 and pME3185 for N- and C-terminal fusions, respectively. For photoreactivation experiments, cryA cDNA (OZG44/45) was cloned in SmaI/NotI sites of pGEX-4T-2 giving pME3187. For overexpression driven by the $A$. nidulans niiA promoter, the same amplicon was inserted into PmeI site of pME3160 resulting in pME3186.

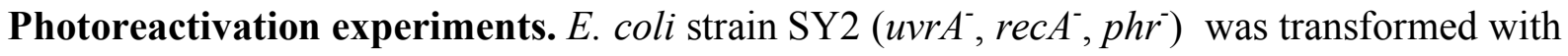
pMS969, pME3187 (cryA cDNA in pGEX-4T-2) and pGEX-4T-2 as an empty control plasmid. Transformants were propagated in LB medium supplemented with $150 \mu \mathrm{g} / \mathrm{ml}$ ampicillin at $37^{\circ} \mathrm{C}$ overnight; expression from pMS969, pME3187 and pGEX-4T-2 was induced by adding IPTG $(0.1 \mathrm{mM}$ final concentration) to the LB medium, and cultures were further incubated for 1 hour with shaking to be spread on LB plates. Plates were irradiated with intensities of 0.3 and $0.6 \mathrm{~J} / \mathrm{m}^{2} \mathrm{UV}$ light (SYLVANIA, G8T5 U.S.A, GERMICIDAL 8W) followed by illumination with $366 \mathrm{~nm}$ UVA-light (SYLVANIA, F8T5/BLB U.S.A, GERMICIDAL $8 \mathrm{~W}$ ). Plates were kept in the dark at $37^{\circ} \mathrm{C}$ overnight and surviving colonies were counted the following day. To test for photoreactivation in A. nidulans, all experiments were performed in a dark room partly illuminated by dim red light. Freshly harvested $A$. nidulans spores were counted and adjusted to $1.5 \times 10^{4}$ for each plate, and spores from strains FGSC A4, cryAA, AGB152-cryAOE, AAH14 and AAH14-cryAOE were spread on Petri dishes $(\varnothing 7 \mathrm{~cm})$ and grown up to 6-7 hours in the darkness to initiate germination. Germinating spores were irradiated with intensities of 448,897 and $1795 \mathrm{~J} / \mathrm{m}^{2} \mathrm{UV}$-light and illuminated with $366 \mathrm{~nm}$ UVA-light for one hour. Treated plates were kept at $30^{\circ} \mathrm{C}$ in the darkness for 3 days, and surviving Aspergillus colonies were counted. For light-dependent development experiments, a custom-made portable illumination chamber equipped with light gradient rooms was utilized. Plates were point-inoculated with $1 \times 10^{5}$ conidia, incubated in light gradient device under $\mathrm{UVA}_{350-370 \mathrm{~nm}}$, blue ${ }_{450 \mathrm{~nm}}$ and $\mathrm{red}_{680 \mathrm{~nm}}$ light or kept in the darkness at $30{ }^{\circ} \mathrm{C}$ for 6 days and the density of number of fruiting bodies was determined by counting the number of cleistothecia per $10 \mathrm{~mm}^{2}$ area.

Fluorescence microscopy. $A$. nidulans spores $\left(0.6 \times 10^{6}\right)$ were either inoculated on $18 \mathrm{~mm} \mathrm{x}$ $18 \mathrm{~mm}$ cover slips submerged in appropriately supplemented liquid medium or on large glass 
slides covered with a thin layer of medium and incubated at $30^{\circ} \mathrm{C}$ overnight. The effect of illumination on CryA localization was investigated by growing selected strains in darkness and light on an agar surface or in submerged culture. Cover slips were mounted on microscope slides using spore storage solution $(0.002 \%$ Tween, $0.5 \% \mathrm{NaCl})$ and fixed with wax. Fluorescence images were taken with a ZEISS Axiovert S100 microscope supported with a HAMAMATSU OCRA-ER digital camera employing the Open $l a b$ tmV5.0.1 software package (IMPROVISION, Coventry, UK). 


\section{Table 1: Strains used in this study}

Strain Genotype Reference

A. nidulans

FGSCA4

Glasgow wild-type

FGSC

AAH14 pabaA1 yA2; $\operatorname{argB2} ; \operatorname{uvsB} \triangle: \because \arg B$

(Hofmann and Harris, 2000)

KHH62 $\operatorname{niiA}(p):: n s d D, v e A+$

(Han et al., 2001)

AGB152 pyroA4, pyrG89, veA+

(Busch et al., 2003)

AGB288 cryA $2:$ ptrA

This study

AGB289 $\quad$ cry $A<n a t^{R}>$

This study

AGB290 $\quad{ }^{p}$ niiA::sgfp::cryA::niiA ${ }^{T}$, A.f. pyrG;

${ }^{p}$ gpdA::mrfp $:: h 2 A,{ }^{p}$ gpdA $:: n a t^{R}$; pyroA4, pyrG89 This study

AGB291 ${ }^{p}$ niiA::cryA::sgfp::niiA ${ }^{T}$, A.f. pyrG;

${ }^{p}$ gpdA ::mrfp $:: h 2 A,{ }^{p}$ gpdA $:: n a t^{R}$; pyroA4, pyrG89 This study

E. coli

SY2 uvrA $A^{-}, r e c A^{-}, p h r^{-}$

(Hitomi et al., 2000)

FGSC: Fungal Genetics Stock Center, Kansas City, MO, USA. 
Table 2: Plasmid constructs utilized in this study

\begin{tabular}{|c|c|c|}
\hline Plasmid & Description & Reference \\
\hline pBluescript II KS & general cloning plasmid [bla, multiple cloning site] & STRATAGENE \\
\hline pGEM5 & general cloning plasmid [bla, multiple cloning site] & PROMEGA \\
\hline pNV1 & $n a t^{R}$ cassette bearing plasmid $[\mathrm{bla}]$ & (Seiler et al., 2006) \\
\hline pGEX-4T-2 & bacterial overexpression plasmid & AMERSHAMBIOSCIENCES \\
\hline pMS969 & E.coli phr (photolyase) overexpression plasmid & (Sancar et al., 1984) \\
\hline pME3181 & cryA::ptrA deletion construct & This study \\
\hline pME3182 & cryA $6.2 \mathrm{~kb}$ genomic fragment & This study \\
\hline \multirow[t]{2}{*}{ pME3183 } & \multicolumn{2}{|c|}{5 UTR $(2 \mathrm{~kb})$ cryA ORF::nat ${ }^{R}:: 3$ UTR in pCR-Blunt II TOPO } \\
\hline & & This study \\
\hline pME3184 & $n$-sgfp $:: c r y A$ under nitrate promoter & This study \\
\hline pME3185 & $c r y A:: s g f p-c$ under nitrate promoter & This study \\
\hline \multirow[t]{2}{*}{ pME3186 } & \multicolumn{2}{|c|}{ cryA overexpression construct, $\operatorname{cry} A$ cDNA under nitrate $\operatorname{promoter}^{p}$ niiA } \\
\hline & & This study \\
\hline \multirow[t]{2}{*}{ pME3187 } & \multicolumn{2}{|c|}{ cryA cDNA in pGEX4-T2 (SmaI / NotI sites) for bacterial expression } \\
\hline & & This study \\
\hline
\end{tabular}


Table 3: Oligonucleotides applied in this study

\begin{tabular}{|c|c|c|}
\hline Designation & Sequence & Feature \\
\hline OZG1 & 5'-ATG GTG AGC AAG GGC GAG GAG CTG-3' & $s g f p-5$ 'start \\
\hline OZG5 & 5'-GAG CTG AGT CAG GCC ACA ACG ATG-3' & cryA 5UTRA \\
\hline OZG6 & 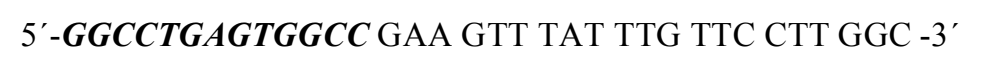 & SfiI-cryA 5UTRB \\
\hline OZG9 & 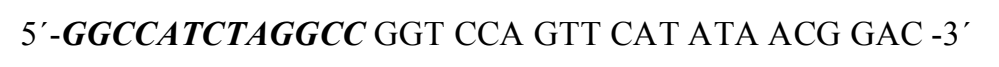 & SfiI-cryA 3UTRA \\
\hline OZG10 & 5'-GCT GCA ACC GCT GCT GCG GCG CC-3' & cryA 3UTRB \\
\hline OZG29 & 5'-CTA CTT GTA CAG TTC GTC CAT GCC GTG-3' & $s g f p-3$ 'end \\
\hline OZG44 & 5'-TTT $\boldsymbol{C} \boldsymbol{C} \boldsymbol{C} \boldsymbol{G} \boldsymbol{G} \boldsymbol{G}$ ATG CGG CAG AAG CGC AAA CTC TCC-3' & $\operatorname{SmaI}$ \\
\hline OZG45 & 5'-TT $\boldsymbol{G} \boldsymbol{C} \boldsymbol{G} \boldsymbol{G} \boldsymbol{C} \boldsymbol{C} \boldsymbol{G} \boldsymbol{C}$ TT ATG CTC CCC GCG CAG CAT CCTT-3' & Not $\mathrm{I}$ \\
\hline \multirow[t]{2}{*}{ OZG87 } & \multicolumn{2}{|c|}{ 5'-GCC ATT GGA GAG TTT GCG CTT CTG CCG CAT ACC ACC GCT ACC } \\
\hline & ACC CTT GTA CAG TTC GTC CAT GCC GTG-3' & $s g f p:: c r y A$ fusion \\
\hline \multirow[t]{2}{*}{ OZG88 } & \multicolumn{2}{|c|}{ 5'-CAT TAA GGA AGT ATA AGG ATG CTG CGC GGG GAG CAG GTG GTA } \\
\hline & GCG GTG GTA TGG GTG G-3' & $\operatorname{cryA}:: s g f p$ fusion \\
\hline OZG89 & $5^{\prime}$-TGC TCC CCG CGC AGC ATC CTT ATA C-3' & $\operatorname{cryA}\left(3^{\prime}\right.$-ex-stop) \\
\hline OZG98 & $5^{\prime}$-CCA TCA GCG AAA CCT CGC GAC AAC-3' & $\operatorname{ros} A$ (NorthernA) \\
\hline OZG99 & 5'-CAG TCC TGA GGT GGA TAG CTG ATA C-3' & $\operatorname{ros} A$ (NorthernB) \\
\hline OZG100 & 5'-GGG ATC ACT AGA GGC TGG ACA TAG-3' & $n s d D$ (NorthernA) \\
\hline OZG101 & 5'-CGC TAA ACT TGA ACG TGA TGG TTC-3' & $n s d D$ (NorthernB) \\
\hline OZG189 & 5'-GGC GGC TCT GAG GTG CAG TGG ATG-3' & $n a t^{R} 5^{\prime}$ \\
\hline \multirow[t]{2}{*}{ OZG191 } & \multicolumn{2}{|c|}{ 5'-GCG AGC AGG CGC TCT ACA TGA GCA TGC CCT GCC CCT GAT ATA } \\
\hline & TAC AGG TCCAGT TCA TAT AAC GG-3' & $\begin{array}{l}3 \text { UTR } n a t^{R} \\
\text { connector }\end{array}$ \\
\hline OZG192 & 5'-TCA GGG GCA GGG CAT GCT CAT GTA GAG-3' & $n a t^{R} 3$ \\
\hline OZG199 & 5'-CCC A $\boldsymbol{A} \boldsymbol{T} \boldsymbol{T} \boldsymbol{A} \boldsymbol{A} \boldsymbol{T}$ TGT ACA AGT ATT ATC AAG GGA CAA G-3' & ' cryA $5 \mathrm{UTR}(V s p \mathrm{I})$ \\
\hline \multirow[t]{2}{*}{ OZG200 } & 5'-CCC A $\boldsymbol{A} \boldsymbol{T} \boldsymbol{T A \boldsymbol { A }} \boldsymbol{T}$ TCC TTA TGC TCC CCG CGC AGC ATC & \\
\hline & CTT ATA $-3^{\prime}$ & $\operatorname{cryA} \mathrm{C}$-ter $(V s p \mathrm{I})$ \\
\hline Sv142 & 5'-ATG GCT ACA CTT GCA GCA CCA CCA-3' & $v e A$ (NorthernA) \\
\hline Sv143 & 5'-TTA ACG CAT GGT GGC AGG CTT TGA GAC C-3' & veA (NorthernB) \\
\hline GPDA1 & 5'-ATG GCT CCC AAG GTC GGA ATC-3' & gpdA (NorthernA) \\
\hline GPDA2 & 5'-CTA TTG GGC ATC AAC CTT GGA G-3' & gpdA (NorthernB) \\
\hline
\end{tabular}




\section{Results}

\section{Aspergillus nidulans encodes a cryptochrome-like protein closely related to class I CPD photolyases}

The cryptochrome/photolyase protein family comprises six sub-groups, which are distinguished according to phylogenetic relationships and functions: class I CPD photolyases, class II CPD photolyases, plant cryptochromes, animal cryptochromes, which include (6-4) photolyases and cry-DASH proteins. All sub-families, except class II CPDs share great sequence homologies. The $A$. nidulans genome reveals only one putative cryptochrome/photolyase gene (AN0387.2, gi 40747330), which we termed cryA. In order to assign this putative cryptochrome/photolyase CryA to one of the subfamilies, several analyses based on multiple sequence alignments with 56 members of the cryptochrome/photolyase family as classified by Daiyasu et al. (Daiyasu et al., 2004) were performed (some data shown) (Fig. 4). Due to their divergence from the other sub-families, the class II CPD photolyases were not included. In the multiple sequence alignments performed with TCOFFEE, ClustalW and MAFFT the deduced cryA sequence showed a high degree of conservation with all other groups (Fig. 4A). Phylogenetic trees were estimated by maximum parsimony, neighbor joining and maximum likelihood methods to result in the expected classification of the reference sequences into the appropriate cryptochrome/photolyase subfamilies. As a general result for all three alignments, the CryA sequence clustered with the class I CPD photolyases (Fig. 4B).

In addition, the multiple alignments were analyzed with nearest-mean and k-nearestneighbour-rule classification methods as well as by principal component analysis (PCA). By means of orthonormal encoding, each position in the alignment was encoded as a 20dimensional vector where each dimension represents one amino acid. In this way, each sequence in the alignment is represented as a vector in a 22.520-dimensional vector space. In order to visualize the positioning of the sequences in the resulting vector space, a dimension reduction by PCA was carried out. Colour coding of the sequences from different subfamilies shows that the subfamilies form separate clusters within this high-dimensional feature space (Fig. 4C). The CryA sequence is located within the group of class I CPD photolyases. For nearest-mean-classification, the mean of the vectors of each subfamily was calculated and the distance to the CryA sequence was analyzed. From the calculations, the nearest mean belonged to the group of class I CPD photolyases with a distance of 0.6982 (CRY-DASH 1.0611, plant CRYs 1.1599, 6-4 photolyases 1.1994, animal CRYs 1.2195). For the k-nearest- 
neighbour-rule classification, the distance of the CryA sequence with respect to all other sequences from the alignment was determined. For $\mathrm{k}=7$, all sequences in the vector space neighbourhood belong to the class I CPD photolyase family. Therefore, all analyses carried out in the present study indicate that in terms of amino-acid sequence CryA is most closely related to class I CPD photolyases.

A

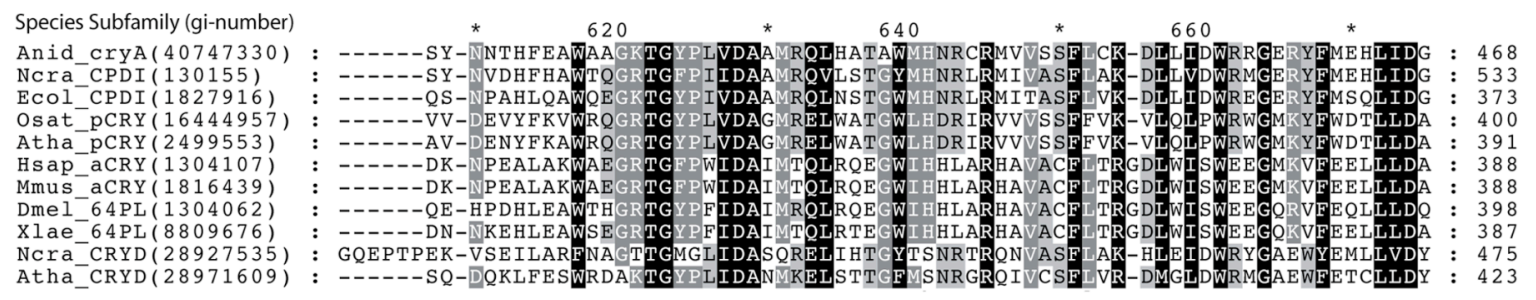

C

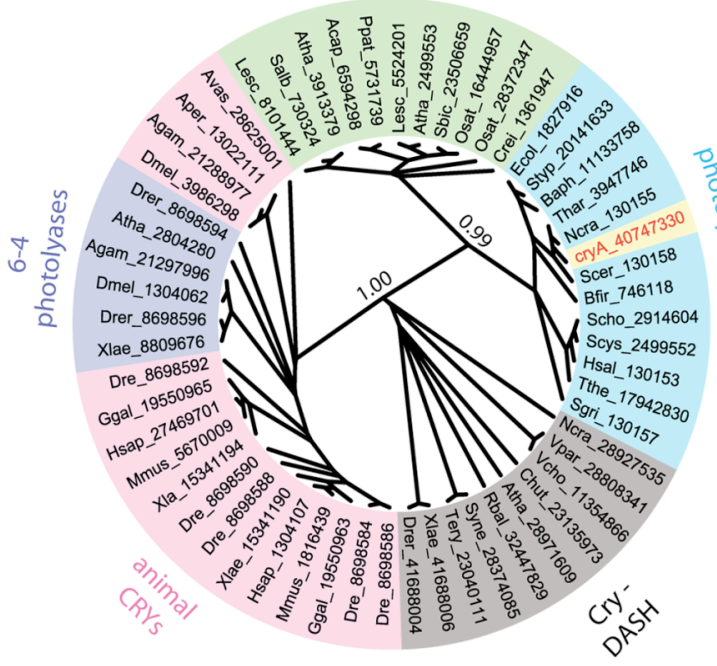

B

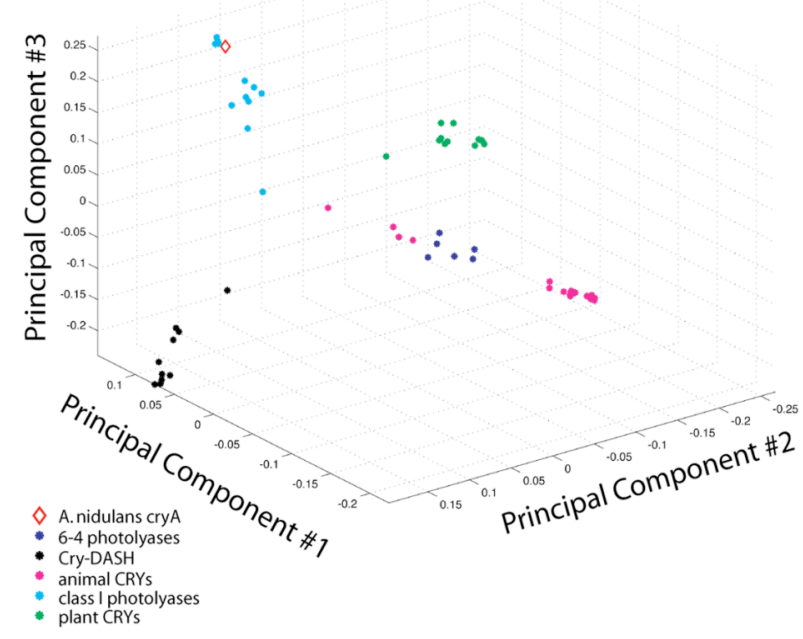

Figure 4: CryA is classified among CPD photolyases. The deduced amino acid sequence of cryA was aligned to 56 previously classified members of the cryptochrome/photolyase family (see (Daiyasu et al., 2004)): six (64)-photolyases, 17 animal cryptochromes, 10 CRY-DASH proteins, 12 class I CPD-photolyases, and 11 plant cryptochromes. Sequence analyses were performed in order to assign CryA to one of these sub-families. (A) Extract of the multiple sequence alignment constructed with T-COFFEE (position 601-675), which illustrates the sequence conservation of CryA and two sequences from each cryptochrome/photolyase subfamily in their FADbinding domain. The degree of sequence conservation is indicated by different shadings: black (100\%), dark grey $(80 \%$ ), light grey $(60 \%$ ), white (less than $60 \%$ ); amino acids of the same similarity group were treated as identical for shading. (B) Radial cladogram to illustrate the phylogenetic relationships of the 56 known cryptochromes/photolyases and CryA. The cladogram represents a consensus tree combining the results of maximum parsimony, neighbor-joining and maximum-likelihood calculations based on the multiple-sequence alignment generated with T-COFFEE. For clarity, bootsrap probabilites are only given for the nodes separating animal CRYs/6-4 photolyases from plant CRYs/class I CPD photolyases (1.00) and plant CRYs from class I CPD photolyases (0.99). (C) Pseudo 3D-representation of the PCA from the ClustalW alignment. The first three principal components of a vector space which represent the subspace with the greatest variance are shown. 


\section{$\operatorname{cry} \boldsymbol{A}$ is expressed in $\boldsymbol{A}$. nidulans late during development}

Since temporal and spatial expression patterns of developmental regulators determine morphogenesis and many other developmental processes that are vital for any organism, we wanted to see how and during which stages the cry $A$ gene is expressed in $A$. nidulans. To this end, first hints were obtained by sequencing of the $c r y A$ cDNA amplified from an A. nidulans cDNA plasmid library. The cryA cDNA amplicon was sequenced and found to lack the intronic region that separates the two exons that encode the DNA photolyase and FAD binding domains on the genomic locus (Fig. 5A). Thus, we concluded that the cryA gene is indeed expressed and that the corresponding pre-mRNA is spliced to yield the mature transcript. Interestingly, the cry $A$ cDNA could be reproducibly amplified from a sexual cDNA library (Krappmann et al., 2006) but not from a vegetative cDNA library (Krappmann et al., 1999). Therefore, cryA mRNA expression was followed in a time course experiment growing the fungus up to 24 hours vegetatively, 12 and 24 hours asexually, and different time periods sexually. Samples of total RNA, which served both as template for the synthesis of PCRcompatible cDNA and RNA for Nothern blot analysis, were prepared from each stage. Results from RT-PCRs (Fig. 5A) and Northern hybridisations (Fig. 5B) were in good agreement to show increased mRNA levels at $24 \mathrm{~h}$ of asexual as well as $48 \mathrm{~h}$ and $72 \mathrm{~h}$ of sexual development.

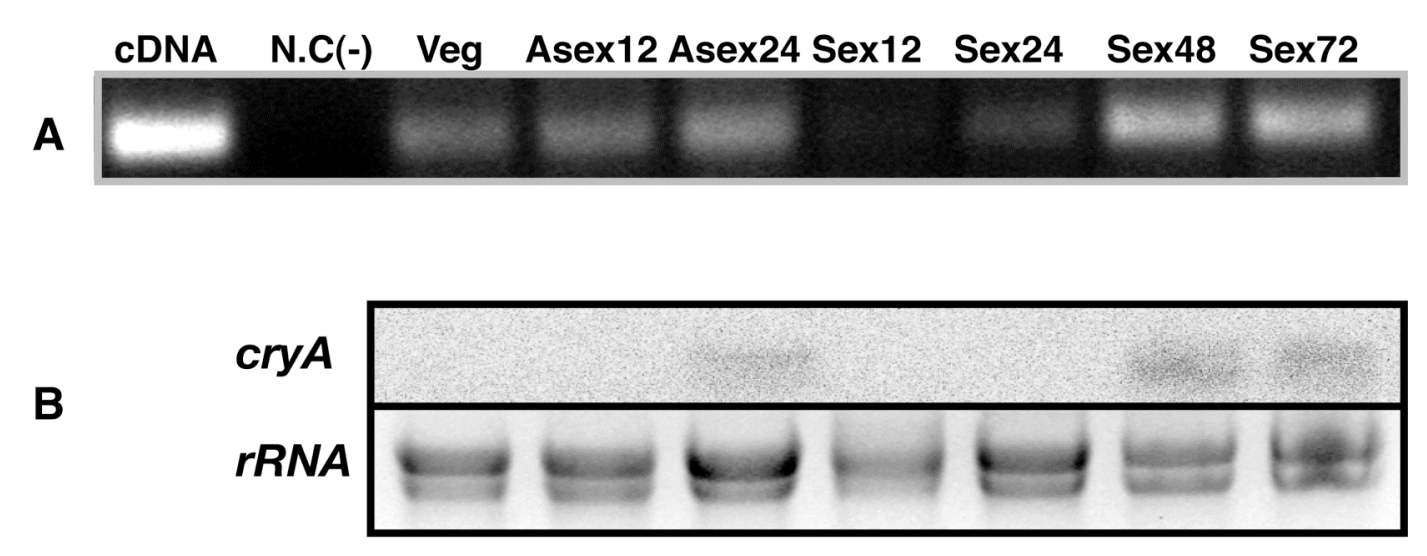

Figure 5: The $\operatorname{cry} \boldsymbol{A}$ gene is expressed during late asexual and sexual developments. $c r y A$ expression was monitored in an A. nidulans wild-type strain by (A) RT-PCR and (B) Northern hybridization experiments. Strain FGSC A4 was vegetatively grown in submerged culture for $24 \mathrm{~h}$ (Veg), transferred onto plates, and kept in the light in order to induce asexual sporulation (Asex) or incubated in the darkness to induce sexual development (Sex). Samples were prepared at the given time points of 12 and 24 hours of asexual and 12, 24, 48, and 72 hours of sexual development. Ethidium bromide rRNA was used as loading control in Northern blots. Signals from RT-PCRs and Northern hybridizations are compatible with each other indicating that $c r y A$ expression is low during vegetative growth and early asexual and sexual development. 
From RT-PCR signals, basal expression can still be observed during vegetative growth and early asexual development. This basal expression increases during late asexual sporulation. Similarly, expression levels remain low during early stages of cleistothecia formation but after 48-72 hours post induction of sexual development, steady-state levels of the cry $A$ transcript increase. Taken together, this expression patterns hint towards a potential role of the cryA gene product during late asexual and sexual differentiation.

\section{Deletion of $\operatorname{cry} A$ results in Hülle cell formation in liquid culture associated with pigment secretion}

Cryptochromes and photolyases were shown to be involved in several cellular functions such as gene regulation, blue-light perception, circadian rhythm entrainment, and DNA photorepair. Therefore, we deleted the cryA gene to address its cellular and presumably photobiological functions with respect to $A$. nidulans development. A suitable deletion cassette was constructed to replace the cryA coding sequence entirely by the selectable marker gene ptrA that confers pyrithiamine resistance. The homologous gene replacement was confirmed by Southern blot analysis (Fig. 6B). Inspection of the resulting cryA-null strain did not show any obvious phenotype in terms of asexual or sexual development under standard growth conditions on solid media. The $c r y A \Delta$ mutant produces similar numbers of asexual spores like the wild-type progenitor and undergoes normal sexual differentiation. Elaborate investigations involving the light response of this strain towards different wave-lengths and doses of light sources did not unveil any abnormality (data not shown). However, when the cryA $\Delta$ and wild-type strains were cultivated submerged in liquid minimal medium, the cry $A \Delta$ strain displayed a distinct phenotype, which is the formation of Hülle cells after 48 hour of growth. While the wild-type strain grows vegetatively and forms long hyphal structures, the mutant forms large numbers of Hülle cells that cover the mycelial balls (Fig. 6C). The formation of cleistothecia was not observed and sexual development remained in an incomplete state in the mutant. Hülle cells are normally formed in the nests that are primordial structures for the formation of $A$. nidulans fruiting bodies where they cover the ripening cleistothecia. The exact function of Hülle cells is still unclear but there are some suggestions for their involvement in protection and nursing. The knock-out phenotype could be successfully rescued by introduction of a $6.5 \mathrm{~kb}$ complementation fragment that was integrated into original locus as confirmed by Southern blot (not shown) (Fig. 6C). The cryA $\Delta$ mutant also exhibits an additional phenotype, which is the secretion of a pink-purple coloured pigment after prolonged incubation in submerged culture. This might be the result of an 
already committed sexual development (Fig. 6D). This phenotype is also complemented by the re-integration of the cryA genomic fragment.

A
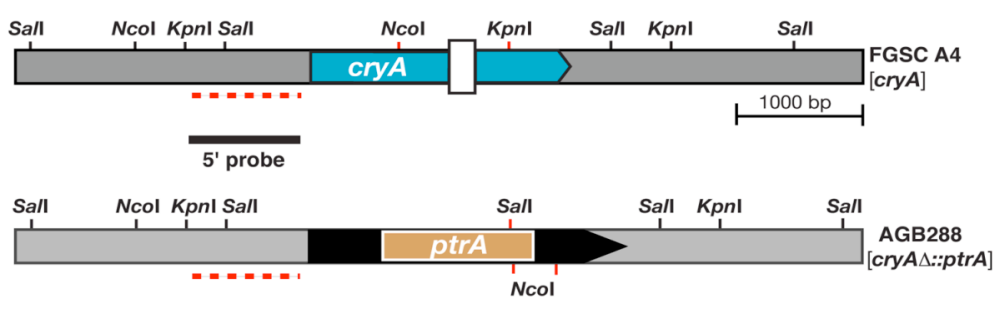

C

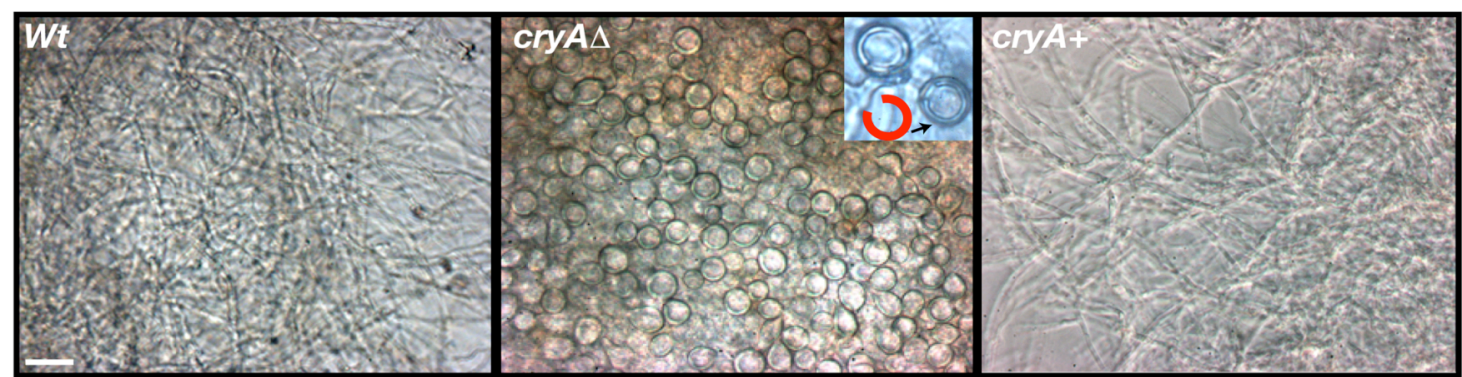

D
B

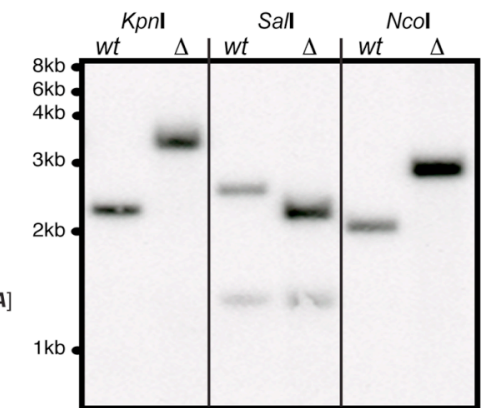

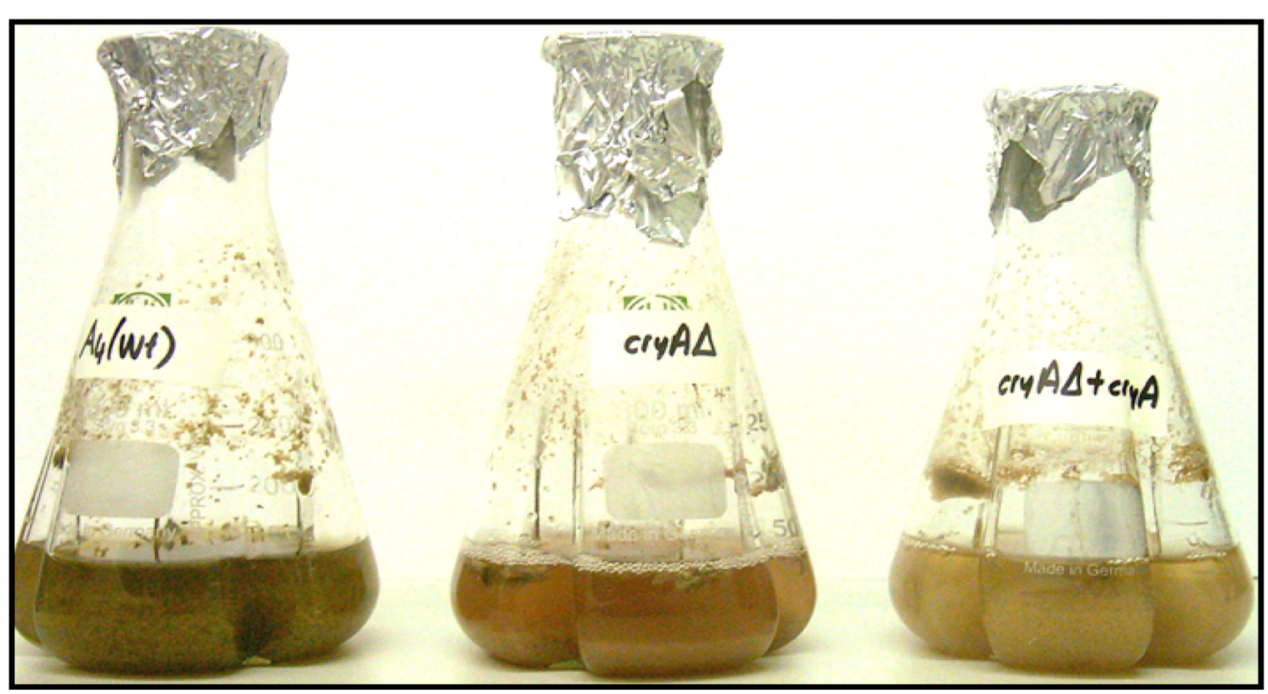

Figure 6: Deletion of $\operatorname{cry} A$ results in Hülle cell formation in submerged culture. (A) Comparative picture of the cryA locus in the wild-type strain A4 and the cryA::ptrA locus in the knock-out strain AGB289. The black bar indicates the region covered by the probe employed in Southern analyses. (B) Autoradiography after Southern hybridization to confirm the deletion of cryA by homologous gene replacement. Signals are compatible with fragments' sizes deduced from theoretical maps. (C) Phenotypical characterisation of cry A deletion strain AGB289 unveils Hülle cell production in submerged culture after 48 hours. Close-up light microscope images illustrate wild-type growth and the development of hyphal mats (left panel) whereas a cryA $\Delta$ mutant produces Hülle cells with their circular, banana-like cell wall shape (middle panel); the deficiency is complemented by integration of a cryA homologous gene replacement fragment (right panel). Pictures were taken after 48 hours of cultivation of strains inoculated with $1 \times 10^{6}$ spores per $\mathrm{ml}$ in submerged culture with shaking in the light at $37^{\circ} \mathrm{C}$. (D) Culture flasks are shown to illustrate the purple-pink pigment production of the cryA $\Delta$ deletion mutant strain in submerged culture. (The white bar represents $20 \mu \mathrm{m}$ ) 
These results suggest that cryA represses sexual development of $A$. nidulans as revealed by the disruption of cryA leading to Hülle cell formation in submerged culture and related to this, secretion of a pink-purple coloured pigment into the culture medium.

\section{CryA is a nuclear protein and represses transcription of the regulators of sexual development}

Most of the cryptochrome/photolyases are nuclear proteins regulating gene expression by an unknown mechanism in both light-dependent and independent manners. For instance, it was demonstrated that the Arabidopsis cryptochromes CRY1 and CRY2 are nuclear proteins regulating blue-light-controlled cell elongation and photoperiodic induction of flowering, respectively whereas CRY3 exhibits a chloroplast localization pattern (Cashmore et al., 1999; Kleiner et al., 1999; Kleine et al., 2003). It was also shown that the fungal WHITE COLLARS, WC-1 and WC-2 are nuclear proteins that shuttle into the nucleus for the entrainment of the circadian clock (Dunlap and Loros, 2004). Conclusively, we wanted to answer the question as to where CryA localizes in the fungal cell and how the localization of the cryA gene product is modulated. For that purpose, we constructed two types of expression cassettes with the cryA cDNA fused $\mathrm{N}$ - or C-terminally to the green fluorescent protein (sGFP) and driven from the nitrogen source-regulated promoter ${ }^{p}$ niiA, which is induced by the presence of nitrate and repressed by ammonium (Muro-Pastor et al., 1999). These constructs were co-transformed in the recipient strain AGB152 together with a $m r f p:: h 2 A$ module to label the nuclei with the monomeric red fluorescent protein (mRFP). Additionally, the sgfp::cryA constructs were transformed into the $\operatorname{cry} A \Delta$ strain to confirm its functionality by mutant phenotype complementation. Both $\mathrm{N}$ - and C-terminal fusion proteins partially restored Hülle cell formation with the former construct complementing to a higher degree (not shown). To see the effect of illumination, we executed the localization experiments in two different conditions darkness and daylight. Despite the fact that no conventional nuclear localization signal (NLS) could be identified in the deduced CryA protein sequence, the sGFP-CryA fusion proteins localize in the nuclei together with the mRFP::H2A fusion along the hypha (Fig. 7). There was no significant difference for the sub-cellular localization of the cry $A$ gene product when the strain was grown in the absence or presence of illumination (not shown). Accordingly, these data demonstrate that the A. nidulans cryptochrome/photolyase-like protein CryA localizes to the nucleus and that its localization is constitutive irrespectively of illumination. 


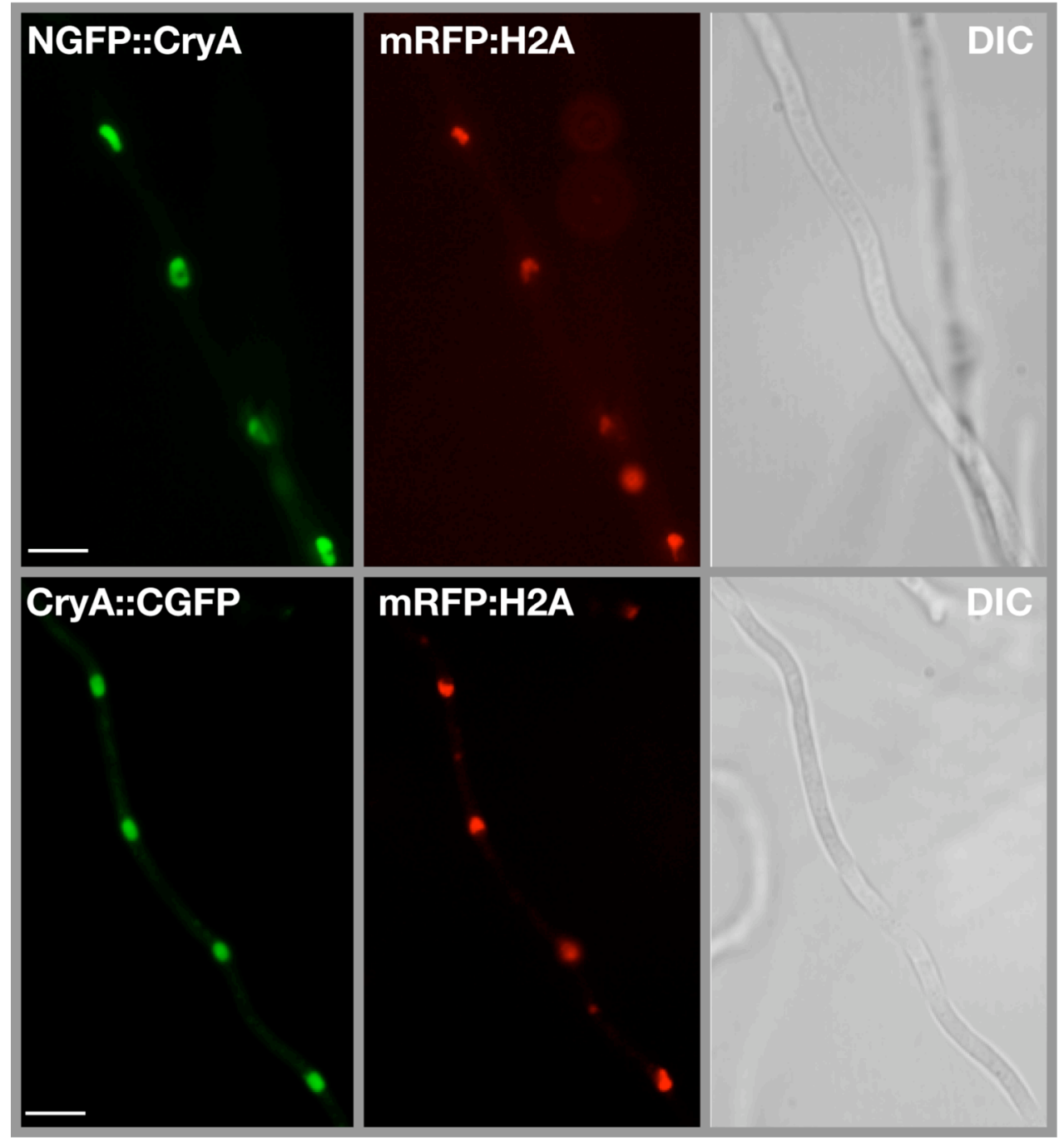

Figure 7: CryA is localized in the nucleus. N- and C-terminal fusions of CryA to sGFP were expressed in order to monitor the subcellular localization of the fusion proteins. To visualize the nuclei in vivo, a mRFP::H2A fusion was utilized. The red fluorescence-containing nuclei line up along the hyphae in accordance with the sGFP signals that demonstrate nuclear localisation of both the chimeric proteins. White bars represent $20 \mu \mathrm{m}$.

The cryptochromes identified to date have been shown to be involved in transcriptional regulation. Conversely, photolyase-like proteins mostly have DNA repair functions and do not operate at the level of transcription. The formation of Hülle cells under inappropriate conditions aroused the question on the conditions that lead to the establishment of these untimely Hülle cells which are normally specific for the sexual developmental programme in $A$. nidulans. When overexpressed, the sexual development regulators veA (velvet $A$ ) and $n s d D$ (never in sexual development $D$ ) cause Hülle cell formation even in submerged culture (Han et al., 2001; Kim et al., 2002). Deletion of the $\mathrm{C}_{2} \mathrm{H}_{2}$ transcription factor rosA (repressor of sexual development $A$ ) results in Hülle cell production in submerged conditions as well (Vienken et al., 2005). Since the cryA $\Delta$ knock-out strain generates Hülle 
cells after 48 hour in submerged growth conditions, we asked how the expression of the developmental regulators, $v e A, n s d D$, and $\operatorname{ros} A$ are regulated in the $c r y A$-null mutant. For that purpose, cryA $\Delta$ mutant and the wild-type strains were grown in liquid culture up to 24,48 and 72 hours of vegetative development. In the mutant, Hülle cells are formed after 48 hours of submerged growth, therefore the transcripts' steady-state levels of these regulators were examined in a time frame before $(24 \mathrm{~h})$ and after $(72 \mathrm{~h})$ this time point (Fig. 8A).

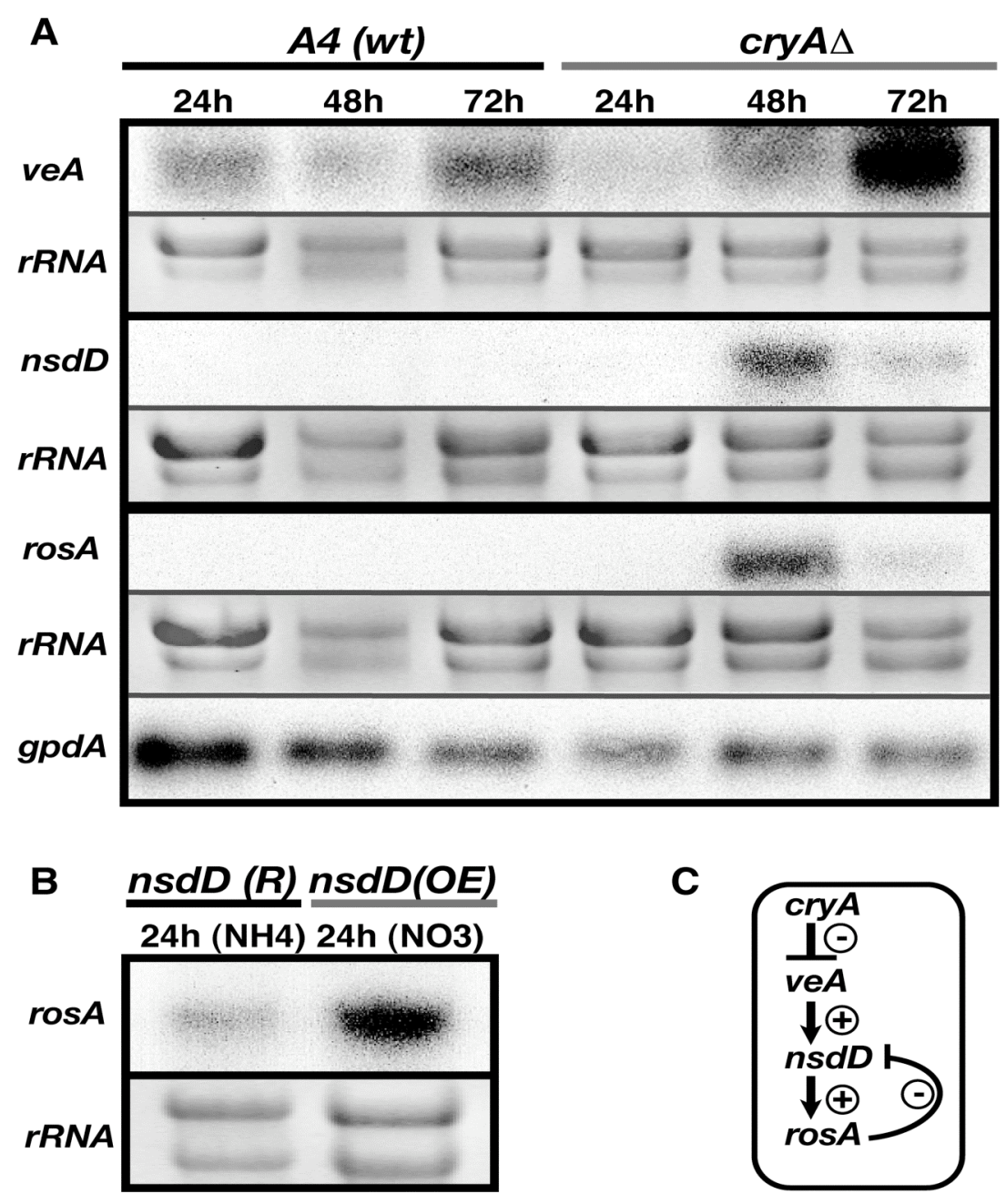

Figure 8: CryA represses the transcripts of regulatory factors of sexual development to reveal a negative feedback loop. (A) Comparative Northern hybridization to monitor transcript levels of selected genes during sexual development in the wild-type strain A4 and the cryA $\Delta$ deletion strain: RNA levels coding for the lightdependent regulator $v e A$ increase in the mutant after 48 hours post induction, corresponding to the appearance of Hülle cells, and increase further up to 72 hours. $n s d D$ and $\operatorname{ros} A$ mRNAs encoding a transcriptional regulators are elevated in the mutant while almost undetectable in A4. As an internal standard, expression of the glycolytic gene $g p d A$ was monitored. (B) $\operatorname{ros} A$ expression is dependent on the expression of the $n s d D$ gene as demonstrated by the overexpression of this gene encoding a regulator of A. nidulans sexual development. (C) Schematic depiction of a negative feedback loop revealed by deletion of cryA gene: deletion of cryA results in elevated levels of $v e A$, which in turn acts positively on $n s d D$ expression; NsdD switches on downstream regulators including its own repressor, the $\operatorname{ros} A$ gene product. 
The transcript encoding the light-dependent activator of sexual development VeA normally shows a faint expression pattern in a wild-type background during vegetative growth whereas $v e A$ expression increases during 48 hour of vegetative growth in the cryA-null strain and further intensifies during 72 hours of growth. Expression of the transcription factor NsdD transcript also escalated during 48 hours of growth corresponding to the observation of Hülle cells but its transcript levels decreased sharply after this time point. mRNA encoding the RosA protein, which is responsible for repressing sexual development under inappropriate conditions, is undetectable during vegetative growth and also accumulates exactly at the time when Hülle cells come into being, which imitates the $n s d D$ expression pattern. As deduced from these expression experiments, transcript levels for two positively-acting sexual development regulators, $v e A$ and $n s d D$ increase in the cry $A \Delta$ strain while remaining at moderate levels in the wild-type strain. On the other hand, expression levels of the negativeacting, transcriptional repressor RosA also elevates in the cryAs strain, which gives some evidence on a presumed co-regulation of these regulators by the $A$. nidulans cryptochrome/photolyase-like protein CryA. Moreover, it had been demonstrated that in a $\operatorname{ros} A \Delta$ strain, which gives rise to Hülle cells in submerged culture, transcripts of $n s d D$ and $v e A$ are up-regulated (Vienken et al., 2005). Since a similar expression pattern could be detected in our Northern hybridisations for $n s d D$ and $\operatorname{ros} A$, we analyzed $\operatorname{ros} A$ mRNA levels in a $n s d D$-overexpression strain (Fig. 8B). The $\operatorname{Ros} A$ transcript levels were only up-regulated in this strain when grown under conditions that forced $n s d D$ expression. Thus, we have found that $n s d D$ over-expression activates $\operatorname{ros} A$ transcription. Moreover, our transcript data support a model in which $v e A$ can be placed upstream of $n s d D$ which activates expression of downstream genes including its own repressor as $\operatorname{ros} A$. The increase in $\operatorname{ros} A$ expression may subsequently cause repression of $n s d D$ and accordingly result in a decrease in $\operatorname{ros} A$ expression implying a regulatory feedback loop (Fig. 8C).

\section{CryA is a blue light sensor for $\mathrm{UVA}_{350-370}$ light}

Light perception is the major function of a typical light receptor. CRYs as blue-light receptors, which are capable of sensing blue-light spectra with different intensities, regulate gene expression from bacteria to fungi to human. In contrast, DNA photolyases only perceive UVA-light for photoreactivation and do not influence gene expression. To test whether a cryA $\Delta$ mutant is impaired in any type of light response, we investigated the cryA $\Delta$ strain under different light sources using blue $450 \mathrm{~nm}$-light and $\mathrm{UVA}_{350-370 \mathrm{~nm}}$-light and red ${ }_{680 \mathrm{~nm}}$-light as a control. To explore a putative interaction with other light regulators, mutant strains 
carrying double deletions such as $\operatorname{cry} A \Delta ;$ veA $\Delta, \operatorname{cry} A \Delta ; \operatorname{csn} D \Delta$ and $\operatorname{cry} A \Delta ;$ fphA $\Delta$ were generated and no apparent phenotype could be detected (not shown). Since CryA resembles a DNA photolyase whose DNA repair feature is activated in E. coli by $\mathrm{UVA}_{366 \mathrm{~mm}}$-light, and regulates gene expression (see below), we looked for any UVA-light-dependent phenotype in the $c r y A \Delta$ strain. Interestingly, the mutant formed more fruit bodies under illumination with UVA $_{350-370 ~ n m}$-light (Fig. 9A, B) and blue ${ }_{454} \mathrm{~nm}^{-}$-light (Fig. 9C) while there is no difference between the cryA mutant and wt under red $_{680 \mathrm{~nm}}$-light (Fig. 9D).

A

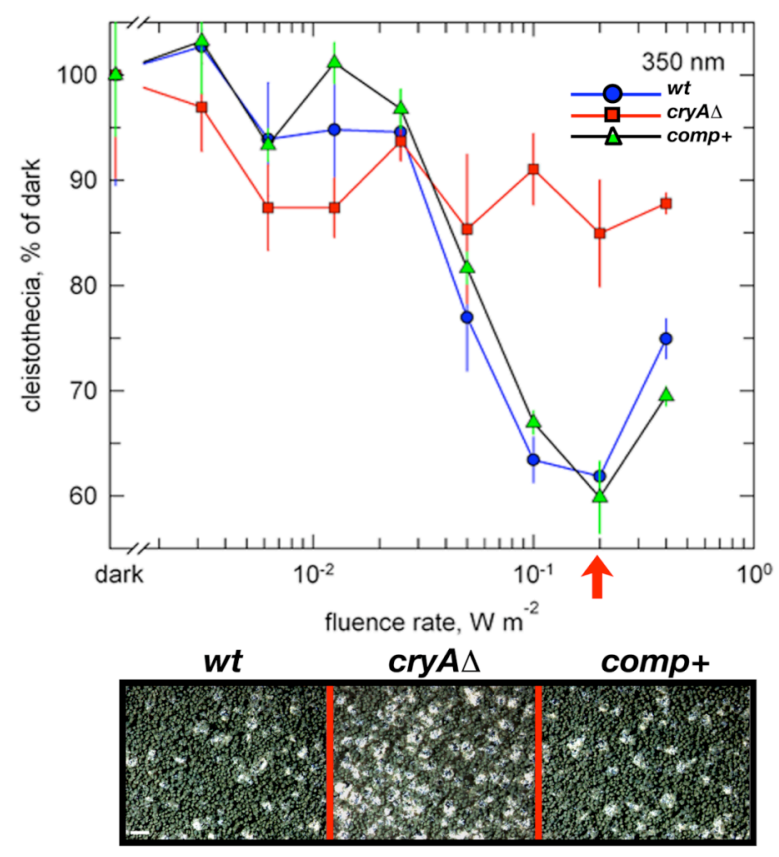

C

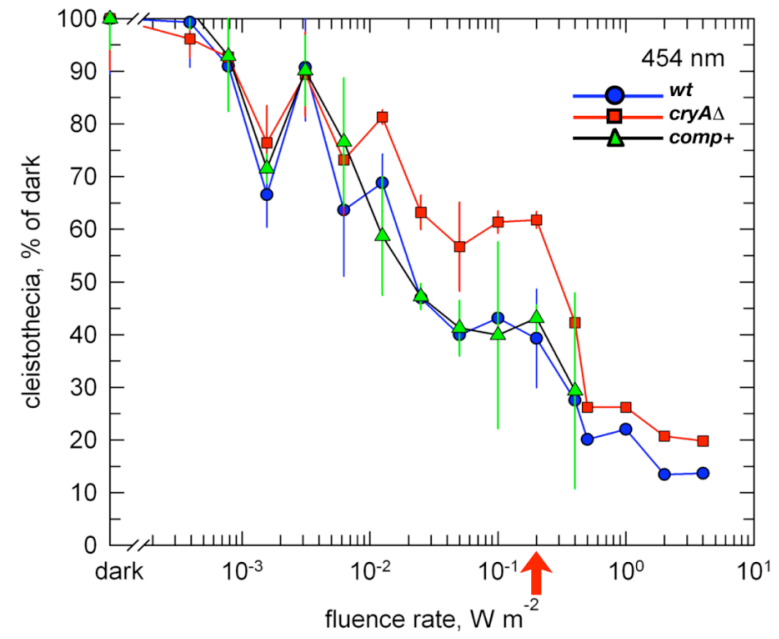

wt

$\operatorname{cry} A \Delta$

comp+
B
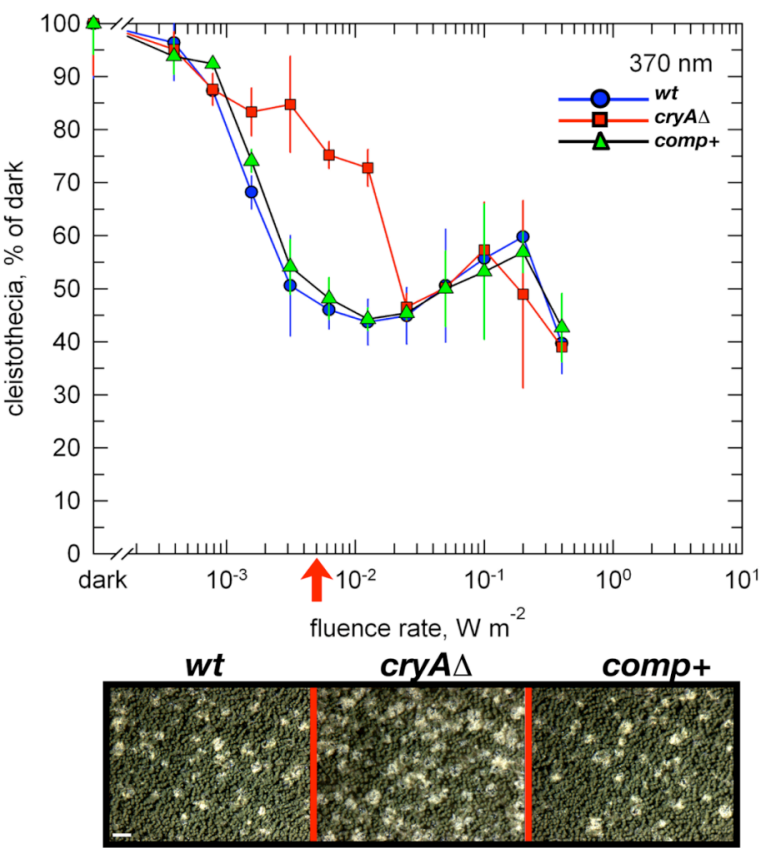

D

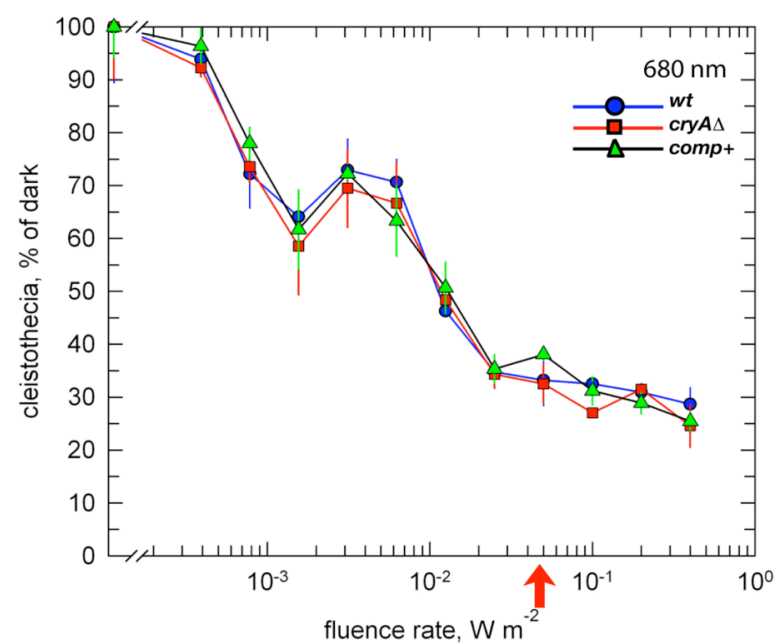

wt

cryA $\Delta$
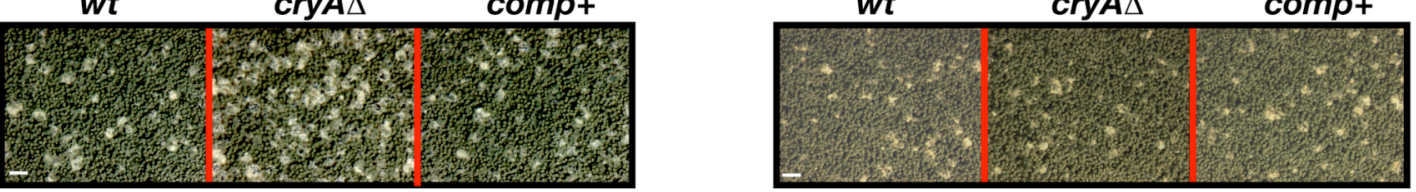
Figure 9: CryA act as a UVA-blue light sensor. Light dose responses of $w t$, cry $A 4$, and complementation strains. (A) At $350 \mathrm{~nm}$ UVA-light, a cryA $\Delta$ knock-out strain forms more sexual structures (lower panel, cleistothecia pictures), (B) At $370 \mathrm{~nm}$ UVA-light, a cryA $\Delta$ knock-out strain is less sensitive to UVA light (lower panel, cleistothecia pictures), (C) Blue light $454 \mathrm{~nm}$ is also partly received by CryA. (D) As a control, at $680 \mathrm{~nm}$ red light spectrum, all the three strains $w t$, cryA $\Delta$ and the complementation strain produce similar number of cleistothecia confirming the UVA light specific sensory function of CryA. The above results are from the densitometric quantification of cleistothecia formation; a $1 \mathrm{~cm}^{2}$ area was cut out from ten different plates and the number of fruiting bodies was counted under binocular microscope. Bars represent $200 \mu \mathrm{m}$.

This finding suggests that cryA has a sexual development repressive function depending on UVA-light and that this effect manifests only under UVA-blue light spectrum implying that the cryptochrome/photolyase-like factor CryA involves in light signalling. Moreover, this indicates that UVA-light, which is usually absorbed by DNA photolyases, somehow regulates the number of cleistothecia via a new class of regulatory protein CryA.

\section{CryA exhibits DNA photorepair activity and confers increased UV-light resistance}

CRYs and photolyases, both being flavoproteins that contain FAD and deazaflavin, are blueor UV-light receptors that display a high degree of similarity. Therefore, it is hard to distinguish a cryptochrome sequence from that of a DNA photolyase by naive inspection of the putative structure or protein domains. When working with cryptochrome/photolyase-like proteins, a valuable functional test is to demonstrate if the studied protein has UV-inducible DNA repair activity. A common method to do so is to express the CRY/photolyase candidate gene in an E. coli host defective in DNA repair and to test whether it rescues the UV sensitivity of this tester strain. To follow this procedure, we cloned the cryA coding sequence in the over-expression plasmid pGEX-4T-2 (a gift from Prof. Dr. Takeshi Todo) and used the construct pMS969 (a gift from Prof. Dr. Aziz Sancar), which contains the native E. coli DNA photolyase $p h r$ gene, as a positive control. Both the expression plasmid pGEX4-cryA and pGEX4-T-2 as empty vector control were transformed into the E. coli recipient strain SY2. Photoreactivation experiments carried out with this set of strains clearly demonstrate that CryA holds a photoreactivated DNA repair activity (Fig. 10A). E. coli strains expressing the endogenous photolyase or the A. nidulans CryA were able to survive irradiation with high doses of UV-light to a greater extent after activation of their repair pathway by illumination with UVA-light. Strains that do not express any photolyase activity or that had not been treated with UVA-light were characterised by low survival rates. These results indicate that the $A$. nidulans cryptochrome/photolyase-like gene $\operatorname{cry} A$ encodes a DNA photolyase activity that complements the phenotype of DNA repair-defective E.coli cells. 
Detecting the photoreversal activity of $A$. nidulans CryA in the DNA repair-deficient E. coli strain, we wondered whether $c r y A$ displays any UV-damaged DNA repair activity in the native host $A$. nidulans. To demonstrate DNA repair function of the CryA protein, we made the use of several strains: wild-type, a cryA $\Delta$ deletion mutant and a $c r y A$ overexpression strain, and strain AAH14, which carries a $u v s B \Delta$ deletion, is deficient for the DNA damage response (Hofmann and Harris, 2000). A distinct number of spores were spread on plates, and after germination at $30^{\circ} \mathrm{C}$, the plates were exposed to doses of UV-light (254 nm) (Fig. 10B).

A

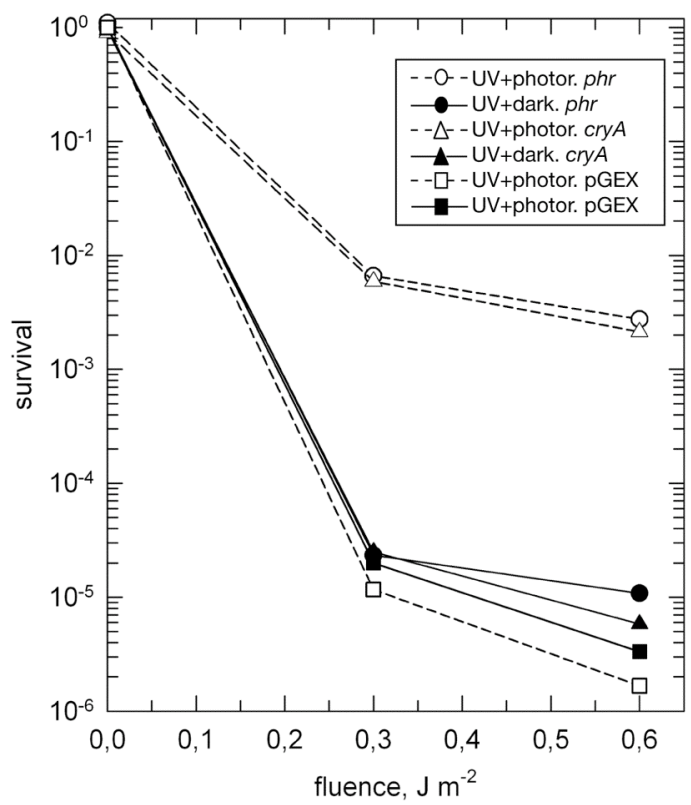

B

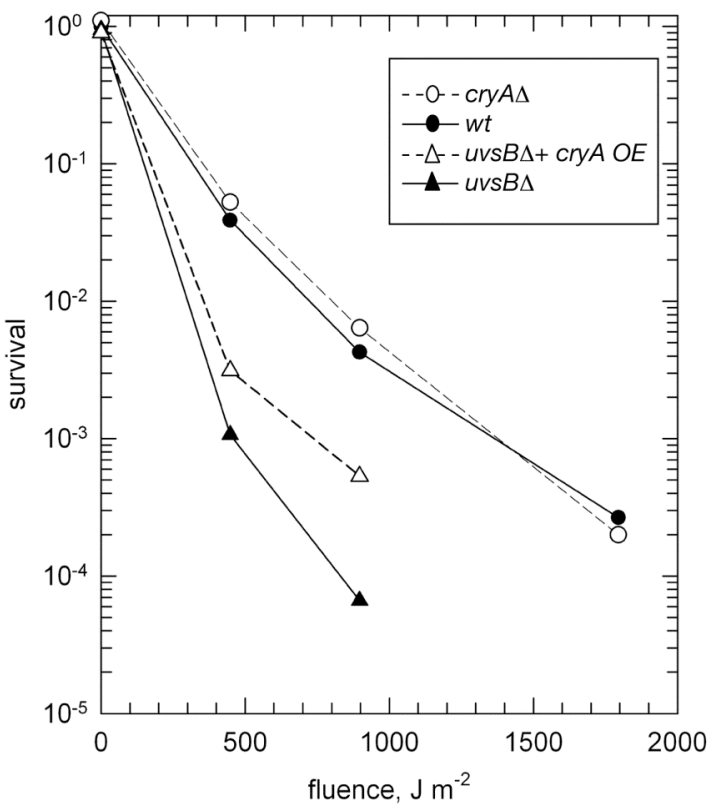

Figure 10: CryA exhibits photoreversal activity. (A) Photoreactivation experiments performed in the repairdefective E. coli strain SY2 ( $\left.u v r A^{-}, r e c A^{-}, p h r^{-}\right)$transformed with the plasmid pGEX-4T-2 as a vector control, pGEX-cryA for CryA expression, or pMS969 as positive control. After UV irradiation, E.coli cells were either kept in the dark (black symbols) or illuminated with $366 \mathrm{~nm}$ light (light symbols). E. coli $\mathrm{Phr}$ and A. nidulans CryA show photoreversal activity after illumination with $366 \mathrm{~nm}$ light for photoreactivation. (B) UV sensitivity experiments performed for A. nidulans; spores of A. nidulans strains A4 (wild-type), AGB288 (cryA4), AAH14 ( $u v s B \Delta$ ) and AAH14 (cryA OE) were exposed to UV light of $254 \mathrm{~nm}$ wavelength with indicated intensities, and the extent of surviving spores was determined by quantification of colony forming units. No difference is evident between A4 and the cryA $\Delta$ deletion mutant whereas UV resistance of strain AAH14 is increased by overexpression of the cryA gene.

After UV illumination of the conidia, plate were illuminated with UVA-light of 366 $\mathrm{nm}$ wave-length for photoreactivation, and eventually plates were incubated either in the dark or at day-light for 3-4 days to assess the number of surviving fungal colonies formed. No UVinduced sensitivity was observed for the $c r y A \Delta$ mutant while the $u v s B \Delta$ strain appeared as highly sensitive towards UV irradiation. However, this phenotype was partially rescued when 
the cryA gene dose was increased by forced overexpression whereas in a wild-type background, cryA overexpression did not result in elevated UV-light resistance (not shown). This suggests that in the wild-type strain, mechanisms such as excision repair might mask the DNA photolyase activity of CryA but in the $u v s B \Delta$ background where these repair mechanisms are inactivated, cryA overexpression increases the resistance to UV irradiation. Taken together, these results from photoreactivation experiments clearly indicate that the CryA cryptochrome/photolyase-like protein confers a pronounced DNA photolyase activity to E.coli and a weak or at least masked DNA photolyase activity to the endogenous host A. nidulans.

Accordingly, all our results propose that the A. nidulans cryA gene encodes a protein possessing a dual function, i.e., DNA photolyase activity and gene regulation. Gene regulation takes place at the level of transcriptional repression of genes encoding key regulators of sexual development. This function is likely to be independent of light perception, as indicated by the constitutive nuclear localization of sGFP::CryA fusion proteins. Moreover, CryA has the ability to transmit a UVA-light signal to the nucleus to repress sexual development under UVA illumination at the identical wavelength that is required for photoreactivation of DNA photolyase activities. Overall, these findings suggest that CryA is an unusual photolyase-like protein that resembles a cryptochrome in terms of regulating gene expression. 


\section{Discussion}

In the presented study, we were able to characterise a chryptochrome/photolyase-like protein encoded by the cryA locus of the filamentous ascomycete $A$. nidulans with respect to its cellular role. In order to determine which sub-family of cryptochrome/photolyases the cry A gene product belongs to, we compared its deduced amino-acid sequence to 56 reference sequences by several methods based on multiple sequence alignments. The reference sequences represent plant and animal cryptochromes, 6-4 photolyases, the so-called CRYDASH proteins, and class I CPD photolyases. In all alignments, CryA showed a high degree of sequence conservation with all subfamilies. This is not surprising considering the great sequence homology commonly found among members of this protein family (Lin and Todo, 2005). The phylogenetic analysis, however, strongly indicates that the CryA sequence is most closely related to class I CPD photolyases. This was independent of the alignment algorithm or phylogeny estimation used in the present study (see Fig. 4C). The phylogenetic analysis was confirmed by independent analyses of the alignment using k-nearest-neighbor rule and nearest-mean classification as well as PCA (Fig. 4B). Therefore, we conclude that the CryA sequence, even though it had been originally retrieved from BLAST searches using cryptochrome sequences as input strings, belongs to the class I CPD photolyase family as it has been defined to date.

To gain a first hint on the cellular function of this protein, a defined deletion mutant strain was generated and phenotypically characterised. The cryA $\Delta$ mutant develops sexual organs, Hülle cells in submerged culture conditions that usually repress sexual and asexual differentiation of $A$. nidulans to maintain vegetative growth. This phenotype has also been described for a mutant strain of $\operatorname{ros} A$, as well as for strains overexpressing sexual regulators $n s d D$ and veA (Han et al., 2001; Kim et al., 2002; Vienken et al., 2005). The veA gene has been isolated and characterized already in 1965 (Kaefer, 1965) but its protein sequence does not reveal any common protein motifs which can explain its function. VeA serves as a positive activator of sexual development and as a negative regulator in asexual development at the same time (Braus et al., 2002a; Kim et al., 2002). Deletion of the veA locus causes a loss of cleistothecia formation while there is increased sexual development and an accumulation of Hülle cells in submerged culture upon veA overexpression. Recently we have shown that $\mathrm{VeA}$ interacts with the master regulator of secondary metabolism thus connecting light-dependent sexual development and secondary metabolite production. The $n s d D$ gene encodes a putative GATA-type transcription factor and has been identified as positive regulator of sexual development and it can induce Hülle cell formation in liquid culture in a 
veA dependent manner upon overexpression (Han et al., 2001). During sexual reproduction $n s d D$ expression is low but shows a significant increase about $12 \mathrm{~h}$ after induction of sexual reproduction, which coincides with the formation of Hülle cells (Han et al., 2001). Since a $\operatorname{ros} A \Delta$ mutant strain also showed an accumulation of Hülle cells in submerged culture conditions and expression analysis revealed an upregulation of $v e A$ and $n s d D$ transcript levels in this genetic background, this putative $\mathrm{Zn}(\mathrm{II})_{2} \mathrm{Cys}_{6}$ transcription factor was proposed as negative regulator of sexual development (Vienken et al., 2005). In fact, the previously mentioned decrease in $n s d D$ expression after induction of asexual reproduction nicely correlates with an increase of $\operatorname{ros} A$ expression and therefore indicates that $\operatorname{ros} A$ downregulates $n s d D$ expression and thereby leads to asexual reproduction (Vienken et al., 2005). $v e A$ transcript levels, on the other hand, gradually increase from $24 \mathrm{~h}$ to $72 \mathrm{~h}$ after the onset of sexual development. A detailed analysis of the transcription data revealed a hierarchical negative feedback loop between activator and repressor elements. Since downstream targets as well as upstream regulators of $n s d D$, veA and $\operatorname{ros} A$ are widely unknown, there are of course many possible ways how this might be explained. In our opinion, a very likely scenario is that cryA normally regulates veA expression negatively in response to light or in a lightindependent manner while $v e A$ positively regulates $n s d D$ expression, which in turn activates expression of its own negative regulator $\operatorname{ros} A$. $\operatorname{ros} A$ does not affect veA expression but represses the transcription of $n s d D$ by preventing sexual commitment at the level of Hülle cells. This implies that $\operatorname{ros} A$ and $n s d D$ constitute a feedback loop which would make sense as $n s d D$ expression seems to be primarily important for the development of Hülle cells, one of the early stages of the sexual life cycle while $v e A$ also is essential for the development of the other sexual organs which are formed subsequently. In response to illumination, VeA changes its nuclear localization. In the light it mostly stays in the cytoplasm and in the darkness, enters the nucleus (Stinnett et al., 2007). FphA (phytochrome) localizes to the cytoplasm and it was suggested that VeA and FphA interacts in yeast two hybrid system (Purschwitz et al., 2006).

The light-independent nuclear localization of CryA indicates that it might regulate gene expression by interacting with other nuclear proteins such as transcription factors, activators or by directly binding to DNA where it represses gene expression crucial for sexual development. As CryA exhibits light-induced photorepair activity in E. coli, it is reasonable to assume that it has the ability to absorb light in the UVA-spectrum to carry out electron transfer reactions. It is substantiated by a cryA $\Delta$ mutant which is less sensitive to UVA-light and produces more cleistothecia under $\mathrm{UVA}_{350-370 \mathrm{~nm}}$-light. These findings propose that in 
addition to light-independent repression of sexual transcription, CryA also possesses a light sensing function that couples the cleistothecia formation to UVA light.

E.coli photoreactivation experiments and the A. nidulans UV survival results indicate that in terms of function, CryA is related to the DNA photolyases. However, its regulatory function is a complete novelty for this class of proteins. An analysis of the photospectroscopic properties of CryA should provide additional hints on the mechanism of CryA function. In summary, we propose that the cryA gene product is a class I CPD photolyase-like protein, a novel member of the class I CPD photolyase subfamily, which localizes in the nucleus independently of light, is a negative regulator of sexual development upon integration of environmental factors, most likely light. We assume that CryA normally represses the veAmediated initiation of a feedback loop between $n s d D$ and $\operatorname{ros} A$ that is important in Hülle cell formation. Furthermore, we speculate that CryA might be the first example of a true dualfunction protein that acts as a photolyase and a cryptochrome. Accordingly, it represents a missing link between the established CRYs and DNA photolyases. Current data collected through photobiological, genetical and biochemical experiments indicate the importance of the so-called white collar genes in blue-light sensing of lower eukaryotes including fungi. In the A. nidulans genome, there are two white collar orthologues that might mediate blue-lightdependent development, which in turn might have caused a slow-down in the evolution of A. nidulans cryptochromes. Repression of the light-independent and $\mathrm{UVA}_{350-370 \mathrm{~nm}}$-dependent sexual development support this hypothesis. Another evolutionary scenario might be that usually most organisms genome encode two or more cryptochrome/photolyase-like genes, some of which have photolyase activity and some have cryptochrome function. The closely related ascomycete $N$. crassa encodes one photolyase and one CRY-DASH protein but in the available genome sequeneces from Aspergilli, only one CRY/photolyase-like protein can be retrieved. In contrast, the basidiomycete Ustilago maydis or Fusarium spp. have more than two CRY/photolyase-like protein encoding genes. It might be possible that some time after the divergence of an Aspergillus progenitor from the Neurospora progenitor, Aspergillus lost the CRY/photolyase-like gene, probably CRY-DASH, through chromosome rearrangements. The loss of the CRY-DASH protein might have caused the endogenous DNA photolyase to gain regulatory functions without losing its DNA photolyase activity, which of course might have slowed down its evolution to a completely functional cryptochrome. Our findings suggest that the characterized photolyase proteins might also possess regulatory functions. 
Chapter 2 


\section{References}

Adams, T.H., Wieser, J.K., and Yu, J.H. (1998). Asexual sporulation in Aspergillus nidulans. Microbiol Mol Biol Rev 62, 35-54.

Blumenstein, A., Vienken, K., Tasler, R., Purschwitz, J., Veith, D., FrankenbergDinkel, N., and Fischer, R. (2005). The Aspergillus nidulans phytochrome FphA represses sexual development in red light. Curr Biol 15, 1833-1838.

Braus, G.H., Krappmann, S., and Eckert, S.E. (2002). Sexual Development in Ascomycetes - Fruit Body Formation of Aspergillus nidulans. In Molecular Biology of Fungal Development, H.D. Osiewacz, ed (New York, Basel: Marcel Dekker, Inc.), pp. 215-244.

Brown, T., and Mackey, K. (1997). Analysis of RNA by Northern and Slot Blot Hybridization. In Current Protocols in Molecular Biology (John Wiley \& Sons Inc.), pp. 4.9.1-4.9.16.

Busch, S., Eckert, S.E., Krappmann, S., and Braus, G.H. (2003). The COP9 signalosome is an essential regulator of development in the filamentous fungus Aspergillus nidulans. Mol Microbiol 49, 717-730.

Cashmore, A.R. (2003). Cryptochromes: enabling plants and animals to determine circadian time. Cell 114, 537-543.

Cashmore, A.R., Jarillo, J.A., Wu, Y.J., and Liu, D. (1999). Cryptochromes: blue light receptors for plants and animals. Science 284, 760-765.

Creevey, C.J., and McInerney, J.O. (2005). Clann: investigating phylogenetic information through supertree analyses. Bioinformatics 21, 390-392.

Daiyasu, H., Ishikawa, T., Kuma, K., Iwai, S., Todo, T., and Toh, H. (2004). Identification of cryptochrome DASH from vertebrates. Genes Cells 9, 479-495. 
Duda, R.O., Hart, P.E., and Stork, D.G. (2001). Pattern classification. (New York ; Chichester England: Wiley).

Dunlap, J.C., and Loros, J.J. (2004). The neurospora circadian system. J Biol Rhythms 19, 414-424.

Feinberg, A.P., and Vogelstein, B. (1983). A technique for radiolabeling DNA restriction endonuclease fragments to high specific activity. Anal Biochem 132, 6-13.

Galagan, J.E., Calvo, S.E., Cuomo, C., Ma, L.J., Wortman, J.R., Batzoglou, S., Lee, S.I., Basturkmen, M., Spevak, C.C., Clutterbuck, J., Kapitonov, V., Jurka, J., Scazzocchio, C., Farman, M., Butler, J., Purcell, S., Harris, S., Braus, G.H., Draht, O., Busch, S., D'Enfert, C., Bouchier, C., Goldman, G.H., BellPedersen, D., Griffiths-Jones, S., Doonan, J.H., Yu, J., Vienken, K., Pain, A., Freitag, M., Selker, E.U., Archer, D.B., Penalva, M.A., Oakley, B.R., Momany, M., Tanaka, T., Kumagai, T., Asai, K., Machida, M., Nierman, W.C., Denning, D.W., Caddick, M., Hynes, M., Paoletti, M., Fischer, R., Miller, B., Dyer, P., Sachs, M.S., Osmani, S.A., Birren, B.W., Eckert, S.E., and Krappmann, S. (2005). Sequencing of Aspergillus nidulans and comparative analysis with $A$. fumigatus and A. oryzae. Nature 438, 1105-1115.

Griffin, E.A., Jr., Staknis, D., and Weitz, C.J. (1999). Light-independent role of CRY1 and CRY2 in the mammalian circadian clock. Science 286, 768-771.

Guindon, S., and Gascuel, O. (2003). A simple, fast, and accurate algorithm to estimate large phylogenies by maximum likelihood. Syst Biol 52, 696-704.

Han, K.H., Han, K.Y., Yu, J.H., Chae, K.S., Jahng, K.Y., and Han, D.M. (2001). The $n s d D$ gene encodes a putative GATA-type transcription factor necessary for sexual development of Aspergillus nidulans. Mol Microbiol 41, 299-309.

Hanahan, D., Jessee, J., and Bloom, F.R. (1991). Plasmid transformation of Escherichia coli and other bacteria. Methods Enzymol 204, 63-113. 
Hitomi, K., Okamoto, K., Daiyasu, H., Miyashita, H., Iwai, S., Toh, H., Ishiura, M., and Todo, T. (2000). Bacterial cryptochrome and photolyase: characterization of two photolyase-like genes of Synechocystis sp. PCC6803. Nucleic Acids Res 28, 2353-2362.

Hofmann, A.F., and Harris, S.D. (2000). The Aspergillus nidulans uvsB gene encodes an ATM-related kinase required for multiple facets of the DNA damage response. Genetics 154, 1577-1586.

Idnurm, A., and Heitman, J. (2005). Light controls growth and development via a conserved pathway in the fungal kingdom. PLoS Biol 3, e95.

Idnurm, A., Rodriguez-Romero, J., Corrochano, L.M., Sanz, C., Iturriaga, E.A., Eslava, A.P., and Heitman, J. (2006). The Phycomyces madA gene encodes a blue-light photoreceptor for phototropism and other light responses. Proc Natl Acad Sci U S A 103, 4546-4551.

Kaefer, E. (1965). Origins of translocations in Aspergillus nidulans. Genetics 52, 217-232.

Kanai, S., Kikuno, R., Toh, H., Ryo, H., and Todo, T. (1997). Molecular evolution of the photolyase-blue-light photoreceptor family. J Mol Evol 45, 535-548.

Katoh, K., Kuma, K., Toh, H., and Miyata, T. (2005). MAFFT version 5: improvement in accuracy of multiple sequence alignment. Nucleic Acids Res 33, 511-518.

Kim, H., Han, K., Kim, K., Han, D., Jahng, K., and Chae, K. (2002). The veA gene activates sexual development in Aspergillus nidulans. Fungal Genet Biol 37, 72-80.

Kleine, T., Lockhart, P., and Batschauer, A. (2003). An Arabidopsis protein closely related to Synechocystis cryptochrome is targeted to organelles. Plant J 35, 93-103.

Kleiner, O., Kircher, S., Harter, K., and Batschauer, A. (1999). Nuclear localization of the Arabidopsis blue light receptor cryptochrome 2. Plant J 19, 289-296. 
Kolar, M., Punt, P.J., van den Hondel, C.A., and Schwab, H. (1988). Transformation of Penicillium chrysogenum using dominant selection markers and expression of an Escherichia coli lacZ fusion gene. Gene 62, 127-134.

Krappmann, S., Jung, N., Medic, B., Busch, S., Prade, R.A., and Braus, G.H. (2006). The Aspergillus nidulans F-box protein GrrA links SCF activity to meiosis. Mol Microbiol 61, 76-88.

Krappmann, S., Helmstaedt, K., Gerstberger, T., Eckert, S., Hoffmann, B., Hoppert, M., Schnappauf, G., and Braus, G.H. (1999). The aroC gene of Aspergillus nidulans codes for a monofunctional, allosterically regulated chorismate mutase. J Biol Chem 274, 22275-22282.

Lin, C., and Shalitin, D. (2003). Cryptochrome structure and signal transduction. Annu Rev Plant Biol 54, 469-496.

Lin, C., and Todo, T. (2005). The cryptochromes. Genome Biol 6, 220.

Liu, Y., He, Q., and Cheng, P. (2003). Photoreception in Neurospora: a tale of two White Collar proteins. Cell Mol Life Sci 60, 2131-2138.

Muro-Pastor, M.I., Gonzalez, R., Strauss, J., Narendja, F., and Scazzocchio, C. (1999). The GATA factor AreA is essential for chromatin remodelling in a eukaryotic bidirectional promoter. Embo J 18, 1584-1597.

Notredame, C., Higgins, D.G., and Heringa, J. (2000). T-Coffee: A novel method for fast and accurate multiple sequence alignment. J Mol Biol 302, 205-217.

Punt, P.J., and van den Hondel, C.A. (1992). Transformation of filamentous fungi based on hygromycin B and phleomycin resistance markers. Methods Enzymol 216, 447457.

Purschwitz, J., Muller, S., Kastner, C., and Fischer, R. (2006). Seeing the rainbow: light sensing in fungi. Curr Opin Microbiol 9, 566-571. 
Saiki, R.K., Bugawan, T.L., Horn, G.T., Mullis, K.B., and Erlich, H.A. (1986). Analysis of enzymatically amplified beta-globin and HLA-DQ alpha DNA with allele-specific oligonucleotide probes. Nature 324, 163-166.

Sambrook, J., Fritsch, E.F., and Maniatis, T. (1989). Molecular cloning: a laboratory manual. (New York: Cold Spring Harbor Laboratory Press).

Sancar, A. (1994). Structure and function of DNA photolyase. Biochemistry 33, 2-9.

Sancar, A., Smith, F.W., and Sancar, G.B. (1984). Purification of Escherichia coli DNA photolyase. J Biol Chem 259, 6028-6032.

Sancar, G.B. (1990). DNA photolyases: physical properties, action mechanism, and roles in dark repair. Mutat Res 236, 147-160.

Seiler, S., Vogt, N., Ziv, C., Gorovits, R., and Yarden, O. (2006). The STE20/Germinal Center Kinase POD6 Interacts with the NDR Kinase COT1 and Is Involved in Polar Tip Extension in Neurospora crassa. Mol Biol Cell 17, 4080-4092.

Selby, C.P., and Sancar, A. (2006). A cryptochrome/photolyase class of enzymes with single-stranded DNA-specific photolyase activity. Proc Natl Acad Sci U S A 103, 17696-17700.

Southern, E.M. (1975). Detection of specific sequences among DNA fragments separated by gel electrophoresis. J Mol Biol 98, 503-517.

Stinnett, S.M., Espeso, E.A., Cobeno, L., Araujo-Bazan, L., and Calvo, A.M. (2007). Aspergillus nidulans VeA subcellular localization is dependent on the importin alpha carrier and on light. Mol Microbiol 63, 242-255.

Swofford, D.L. (2003). PAUP*. Phylogenetic Analysis Using Parsimony(*and other methods). (Sunderland, Massachusets: Sinauer Associates). 
Thompson, J.D., Higgins, D.G., and Gibson, T.J. (1994). CLUSTAL W: improving the sensitivity of progressive multiple sequence alignment through sequence weighting, position-specific gap penalties and weight matrix choice. Nucleic Acids Res 22, 4673-4680.

Trooskens, G., De Beule, D., Decouttere, F., and Van Criekinge, W. (2005). Phylogenetic trees: visualizing, customizing and detecting incongruence. Bioinformatics 21, 3801-3802.

Vienken, K., and Fischer, R. (2006). The Zn(II)2Cys6 putative transcription factor NosA controls fruiting body formation in Aspergillus nidulans. Mol Microbiol 61, 544554.

Vienken, K., Scherer, M., and Fischer, R. (2005). The Zn(II)2Cys6 putative Aspergillus nidulans transcription factor repressor of sexual development inhibits sexual development under low-carbon conditions and in submersed culture. Genetics 169, 619-630.

Yasui, A., Eker, A.P., Yasuhira, S., Yajima, H., Kobayashi, T., Takao, M., and Oikawa, A. (1994). A new class of DNA photolyases present in various organisms including aplacental mammals. Embo J 13, 6143-6151. 


\title{
Chapter 3
}

\section{Neurospora crassa ve-1 affects asexual conidiation}

\begin{abstract}
The velvet factor of the homothallic fungus Aspergillus nidulans promotes sexual fruiting body formation. The encoding veA gene is conserved among fungi, including the ascomycete Neurospora crassa. There, the orthologous ve-1 gene encodes a deduced protein with high similarity to $A$. nidulans VeA. Cross-complementation experiments suggest that both the promoter and the coding sequence of $N$. crassa ve-1 are functional to complement the phenotype of an A. nidulans deletion mutant. Moreover, ve-1 expression in the heterologous host A. nidulans results in development of reproductive structures in a light-dependent manner, promoting sexual development in the darkness while stimulating asexual sporulation under illumination. Deletion of the $N$. crassa ve-1 locus by homologous gene replacement causes formation of shortened aerial hyphae accompanied by a significant increase in asexual conidiation, which is not light-dependent. Our data suggest that the conserved velvet proteins of A. nidulans and N. crassa exhibit both similar and different functions to influence development of these two ascomycetes.
\end{abstract}




\section{Introduction}

Light is an essential source of energy that drives life on earth. Animals, plants, fungi, and cyanobacteria exploit the various spectra of light in order to survive. Light can cause many behavioural and developmental changes in metabolism, growth, directionality, or morphogenesis, respectively (Gyula et al., 2003). In addition, light is a trigger for adjusting diurnal duration or circadian rhythms, which can be found from cyanobacteria to fungi to humans (Bell-Pedersen et al., 2005). Among living organisms, fungi occupy an important position to study light and its effects on development in a straight-forward and effortless manner. Light-driven experiments with different groups of fungi have demonstrated that fungi are responsive to a broad spectral range of light, among which blue and red light display prominent effects on fungal growth (Mooney and Yager, 1990; Kües, 2000; Cerda-Olmedo, 2001; Liu et al., 2003; Idnurm and Heitman, 2005; Purschwitz et al., 2006).

The two model organisms, Neurospora crassa and Aspergillus nidulans, which belong to the ascomycetes, are distantly related fungi that exhibit different responses to the light spectrum. N. crassa is more receptive to blue light while $A$. nidulans responds primarily to red light illumination (see below). Both fungi are suitable to study molecular mechanisms leading to development and cell differentiation. They are predominantly haploid during their life cycle, which makes them preferable to investigate and observe the interaction between genes and development. In submerged culture, $N$. crassa and $A$. nidulans produce a web of branched vegetative hyphae called mycelium. When exposed to an aerial interface, mycelia differentiate and form asexual spores termed conidia. Two types of asexual spores are produced by N. crassa: multinucleate macroconidia and uninucleate microconidia (Siegel et al., 1968; Springer, 1993; Maheshwari, 1999). Conidiation is induced primarily by dessication, carbon source limitation as well as light and changes in $\mathrm{CO}_{2}$ partial pressure. A phlethora of conidiaspecific genes has been described (reviewed by (Springer and Yanofsky, 1989). In A. nidulans, mycelia grown on a solid and illuminated surface develop asexual sporeproducing structures called conidiophores. This is initiated by a foot cell that swells and gives rise to layers of metulae, which in turn result in the phialide cells producing chains of asexual conidia by consecutive budding (Adams et al., 1998). Every stage of this conidiophore formation is controlled by temporal and spatial expression of transcription factors that are specific for each developmental stage, such as BrlA, AbaA, and WetA (Adams et al., 1988; Marshall and Timberlake, 1991; Andrianopoulos and Timberlake, 1994).

Sexual differentiation in ascomycetes results in fruiting bodies of different shapes, depending on the taxonomical group. The homothallic fungus $A$. nidulans forms sexual 
fruiting bodies called cleistothecia that are spherical, closed structures enveloping the asci (reviewed by Braus et al., 2002). N. crassa produces bottle-shaped sexual architectures named perithecia, and sexual development of this fungus is controlled by two mating type genes (Pöggeler et al., 2006). Nitrogen limitation causes the formation of protoperithecia which serve as recipient for male nuclei of the opposite mating type, and fertilized protoperithecia develop into perithecia in which eight linearly-ordered homokaryotic ascospore containing asci are formed. Sexual development in A. nidulans can also occur either by mating of two strains but also via selfing in the absence of a partner. In this ascomycete, sexual development takes place following asexual sporulation and is initiated by the formation of specific mycelial aggregates formed by so-called Hülle cells. Within these nests, the spherical products of sexual differentiation develop that contain meiotically derived, red-pigmented ascospores (Braus et al., 2002b; Sohn and Yoon, 2002).

Developmental programmes such as spore formation are often subject to various environmental stimuli, among them illumination. Especially the blue light responses of $N$. crassa have been widely studied: blue or UV light stimulates carotenoid synthesis, induction of protoperithecia and their prototropism, asexual spore formation, hyphal growth induction and entrainment of the circadian clock (Linden et al., 1997). Many of these responses are regulated through the phototropin-like proteins, WHITE COLLAR-1 (WC-1) and WHITE COLLAR-2 (WC-2), the former being the first fungal photoreceptor described (Ballario et al., 1996; Linden and Macino, 1997; Froehlich et al., 2002; He et al., 2002). A second type of blue light receptor called VIVID (VVD), which contains a sensory light-oxygen- $\underline{\text { voltage }}$ (LOV) domain that binds flavins like FAD or FMN, is presumed to sense light intensity changes (Crosson et al., 2003; Schwerdtfeger and Linden, 2003).

In contrast, A. nidulans shows a distinct red light response. For A. nidulans, illumination negatively affects fruiting body formation as it inhibits sexual development and promotes the asexual developmental programme. When incubation is carried out in the dark, increased numbers of fruiting bodies are formed; on the other hand, growth in day light conditions or under red light illumination leads to asexual sporulation (Zonneveld, 1977). Recently, a phytochrome red light receptor encoded by the fphA gene was shown to be responsible for repression of sexual fruiting body formation and induction of conidiation under red light conditions (Blumenstein et al., 2005). The so-named velvet factor, firstly described by (Käfer, 1965), plays a prominent role in light-dependent development of A. nidulans: veAl mutants show retarded sexual development and excessive asexual sporulation. Later it was demonstrated that in a wild-type $v e A^{+}$background red light promotes 
the formation of conidiospores, while veAl strains are not affected by this kind of illumination (Mooney and Yager, 1990). Therefore, the veA gene product was proposed to be a negative regulator of asexual conidiation whose function could be suppressed by red or day light (Champe et al., 1981; Mooney and Yager, 1990; Timberlake, 1990; Yager, 1992). The encoding veA locus was cloned and preliminary characterized, demonstrating that deletion of the gene results in an acleistothecial phenotype even under conditions that promote sexual development (Kim et al., 2002). Accordingly, veA overexpression results in Hülle cell formation even in submerged culture. This work also demonstrated that the veAl allele was formed as a result of G-to-T nucleotide substitution in the start codon of the wild-type veA gene leading to downstream translational initiation and synthesis of a truncated gene product. Moreover, VeA's role seems to be not only restricted to sexual development in Aspergillus species, as it was also shown that the veA gene product of $A$. parasticus affects sclerotial production as well as secondary metabolism (Calvo et al., 2004) and that a veA gene disruption in the asexual pathogen A. fumigatus reduces asexual sporulation in dependency of the nitrogen source (Calvo et al., 2004; Krappmann et al., 2005). Moreover, recent data suggest that the Fusarium verticilloides velvet orthologue FvVE1 regulates filamentous growth and the ratio of microconidia to macroconidia, and that velvet-like proteins are conserved throughout the fungal kingdom (Li et al., 2006).

The genome sequences of $N$. crassa and A.nidulans have been determined (Galagan et al., 2003; Galagan et al., 2005a), and inspection of both genomes reveals many orthologous sequences, whose function may be conserved or altered during the course of evolution. In this report we show that the $N$. crassa genome contains one copy of a velvet gene orthologue encoding a protein that shares $50 \%$ similarity to that of $A$. nidulans, VeA. The locus was targeted via homologous gene replacement to uncover that deletion of the $N$. crassa ve- 1 gene results in shortened aerial hypha formation accompanied by increased conidiation, which can be complemented by re-introduction of a $7 \mathrm{~kb}$ genomic fragment comprising ve-1. Crosscomplementation experiments indicate that the $N$. crassa ve-l gene rescues the phenotype of an $A$. nidulans veA $\Delta$ deletion strain and that it is regulated in the same manner like veA in the exogenous host, supporting a functional as well as structural conservation of the velvet factor among these two ascomycetes. 


\section{Materials and Methods}

\section{Strains, media, and growth conditions}

Fungal strains used during this study are listed in Table 4. N. crassa strain FGSC987 (74-OR23-1A) served as recipient for the ve-1 deletion cassette and for over-expression experiments; A. nidulans strain DVAR1 was used for cross-complementation experiments. Standard laboratory Escherichia coli strains DH5 $\alpha$ and MACH-1 (INVITROGEN) were used for preparation of plasmid DNA and were propagated in LB medium (1\% tryptone, $0.5 \%$ yeast extract, $1 \% \mathrm{NaCl})$ supplemented with $100-150 \mu \mathrm{g} \cdot \mathrm{ml}^{-1}$ ampicillin. Minimal medium $\left(0.52 \mathrm{~g} \cdot \mathrm{l}^{-1}\right.$ $\mathrm{KCl}, 0.52 \mathrm{~g} \cdot \mathrm{l}^{-1} \mathrm{MgSO}_{4}, 1.52 \mathrm{~g} \cdot \mathrm{l}^{-1} \mathrm{KH}_{2} \mathrm{PO}_{4}, 0.1 \%$ trace element solution, $\mathrm{pH}$ 6.5) was used for growth of $A$. nidulans strains (Käfer, 1965), supplemented with appropriate amounts of 4-aminobenzoic acid (PABA, $1 \mu \mathrm{g} \cdot \mathrm{ml}^{-1}$ ), nourseothricin-dihydrogen sulfate (100$120 \mu \mathrm{g} \cdot \mathrm{ml}^{-1}$ ) (clonNAT, WERNER BIOAGENTS), pyrithiamine (TAKARA Bio Inc.) $\left(0.1 \mu \mathrm{g} \cdot \mathrm{ml}^{-1}\right) ; 1 \%$ D-glucose was used as the source of carbon together with $10 \mathrm{mM}$ ammonium or nitrate as nitrogen source. $N$. crassa strains were grown in either liquid or solid (supplemented with $2 \%$ agar) Vogel's minimal media with $1.5 \%$ sucrose, and as selective agents nourseothricin-dihydrogen sulfate $\left(35 \mu \mathrm{g} \cdot \mathrm{ml}^{-1}\right)$ (clonNAT, WERNER BIOAGENTS) or hygromycin (INVIVOGEN) $\left(200 \mu \mathrm{g} \cdot \mathrm{ml}^{-1}\right.$ ) were added. SC media was prepared as described by (Davis and DeSerres, 1970), composition of cornmeal agar media was $2 \%$ cornmeal agar (SIGMA) containing $0.1 \%$ glucose. For light response experiments, a portable self-made illumination chamber equipped with blue and red lights of $470 \mathrm{~nm}$ and $630 \mathrm{~nm}$ wavelengths, respectively, was used.

\section{Transformation procedures}

E. coli cells were transformed using calcium- and manganese-treated cells (Hanahan et al., 1991). A. nidulans was transformed by polyethylene glycol-mediated fusion of protoplasts described in detail by (Punt and van den Hondel, 1992). Transformation of N.crassa spheroplasts was performed essentially as described earlier by (Vollmer and Yanofsky, 1986).

\section{Recombinant DNA procedures and hybridisation techniques}

For recombinant DNA technology, standard protocols were performed (Sambrook et al., 1989b). Taq, Pfu (MBI Fermentas) and, for long templates, Platinum Taq DNA high fidelity polymerase (INVITROGEN) were used in polymerase chain reactions (Saiki et al., 1986), and crucial cloning steps were validated by sequencing on an ABI Prism 310 capillary sequencer (PE BIOSYSTEMS). Fungal genomic DNAs were prepared from ground mycelia 
(Kolar et al., 1988), and hybridisation analyses using nylon membranes were conducted essentially as described (Southern, 1975) using the OZG20/32 amplified ve-1 5' UTR flanking region as a probe. Total RNA samples were purified using the TRIzol reagent (INVITROGEN) followed by Northern hybridization according to the protocols cited by (Brown and Mackey, 1997). The StRATAgene Prime-It II kit was utilized to radioactively label hybridization probes in the presence of $\left[\alpha_{-}{ }^{32} \mathrm{P}\right] \mathrm{dATP}$ (Feinberg and Vogelstein, 1983). To produce autoradiographies, washed membranes were exposed to KODAK X-Omat films. Quantification of signal intensities was carried out employing a Bio-Imaging Analyzer from Fuji Photo Film Co. LtD. Sequence data were analysed using the LASERGENE software package from DNASTAR, and protein sequence alignments were created by the Clustal W method (http://www.ebi.ac.uk/clustalw/index.html; (Thompson et al., 1994). Mapping and alignment of promoter regions for transcription factor binding sites was performed via the web server http://genome.imim.es/software/meta/meta.html (Blanco et al., 2006).

\section{Plasmid constructions}

The plasmid constructs created and used throughout this study are listed in Table 5; oligonucleotide sequences are given in Table 6. A 5' UTR flanking region of the $N$. crassa ve-1 gene was amplified with OZG21/22 and cloned into the SmaI site of pBluescript KS II (STRATAGENE), and the OZG19/20-amplified 3' UTR of ve- 1 was cloned into the EcoRV site of this plasmid. Finally, the $h p h$ marker gene conferring hygromycin resistance was amplified using pMP6 as template with OZG17/18, digested with SfiI and inserted into the SfiI site between the flanking regions yielding pME3174. A $7.4 \mathrm{~kb}$ genomic fragment (SspI) of the $N$. crassa ve-1 locus was cloned in EcoRV of pBluescript KS II giving pME3175; from this, a $7.2 \mathrm{~kb}$ fragment was released with ApaI and inserted into pNV1 (ApaI) to yield pME3176, which was used for complementation of NCOB1. The $5 \mathrm{~kb}$ genomic fragment of $v e-1$ of $N$. crassa was amplified with OZG47/48, digested with ApaI and ligated into the ApaI site of pNV1 to result in pME3177. To create the pME3179 chimeric plasmid, pME3178 was digested with $X b a \mathrm{I}$ and $B g l \mathrm{II}$ to remove the veA coding sequence, and the OZG36/37amplified, $X b a \mathrm{I}-B g l \mathrm{II}$ digested $v e-1$ ORF was inserted yielding the ve-1 gene driven from the A. nidulans veA promoter. The pME3180 chimeric construct was generated by digesting pME3177 with SpeI/PmiI and inserting OZG49/50-amplified and SpeI/PmiI-digested cDNA of $v e A$, which had been amplified from the sexual stage-specific cDNA plasmid library pCNS4 (Krappmann et al., 2006). 
Table 4: Fungal strains used in this study

\section{Strain Genotype}

\section{Reference}

Neurospora crassa

wild-type FGSC987 (74-OR23-1A)

FGSC

NCOB1

ve-1 $1 \Delta:: h p h$

This study

NCOB2

${ }^{p}$ ve-1::ve-1::ve-1 ${ }^{t},{ }^{p}$ gpdA $:: n a t^{R}$, ve-1 $1::$ hph

This study

Aspergillus nidulans

DVAR1 pabaA1, $y A 2 ; \arg B \Delta:: \operatorname{trp} C ; \operatorname{trp} C 801 ; \operatorname{veA} \Delta:: \arg B$

(Kim et al., 2002)

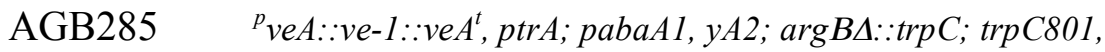
veA $\Delta:: \arg B$

This study

AGB286 ${ }^{p} v e-1:: v e A:: v e-1^{t},{ }^{p}$ gpdA::nat ${ }^{R} ;$ pabaA1, yA2; $\arg B \Delta:: \operatorname{trp} C$; $\operatorname{trp} C 801, \operatorname{ve} A \Delta:: \arg B$

This study

AGB287 ${ }^{p}$ ve-1::ve-1::ve-1 ${ }^{t},{ }^{p}$ gpdA::nat ${ }^{R} ;$ pabaA1, yA2; $\arg B \Delta:: \operatorname{trp} C$;

$\operatorname{trp} C 801, \operatorname{ve} A \Delta:: \arg B$

This study

Table 5: Plasmid constructs employed in this study

\begin{tabular}{|c|c|c|}
\hline Plasmid & Description \& Characteristics & Reference \\
\hline pMP6 & ${ }^{p}$ cpc-1::hph::trpC $C^{t}$ bla & (Seiler et al., 2006) \\
\hline pBluescript II K & general cloning plasmid [bla, multiple cloning site] & STRATAGENE \\
\hline pPTRII & autonomously replicating Aspergillus plasmid $[p \operatorname{tr} A, A M A 1, b l a]$ & TAKARA \\
\hline pNV1 & $n a t^{R}$ plasmid conferring resistance for clonNAT & (Seiler et al., 2006) \\
\hline pME3174 & $v e-1$ deletion cassette $[v e-1: \because h p h]$ & This study \\
\hline pME3175 & $7.4 \mathrm{~kb} v e-1$ locus (SspI) subcloned in EcoRV of pBluescript II KS & This study \\
\hline pME3176 & $7.2 \mathrm{~kb} v e-1$ genomic locus in ApaI site of $\mathrm{pNV} 1$ & This study \\
\hline pME3177 & $5 \mathrm{~kb} v e-1$ locus in ApaI site of $\mathrm{pNV} 1$ & This study \\
\hline pME3178 & $4.6 \mathrm{~kb}$ veA HindIII locus with ptrA resistance in pBluescript II KS & This study \\
\hline pME3 179 & $\operatorname{chimeric}^{p} v e A: \because v e-1: \because v e A^{t}$ construct & This study \\
\hline pME3180 & chimeric $^{p} v e-1: \because v e A: \because v e-1^{t}$ construct & This study \\
\hline
\end{tabular}


Table 6: Oligonucleotides utilized in this study

\begin{tabular}{|c|c|c|}
\hline Designation & Sequence & Restriction site \\
\hline OZG17 & 5'- ggccactcaggcc GAA TTC AAC TGT CCG ATA TCG GTA -3' & $S f i \mathrm{I}$ \\
\hline OZG18 & 5'- ggcctagatggce TCT AGA AAG AAG GAT TAC CTC TAA AC-3 & 'SfiI \\
\hline OZG19 & $5^{\prime}$ - GTC GAC GAC TTT GTT GGT TGG CAT TCA CCG -3' & \\
\hline OZG20 & 5'- ggcetgagtggcc GGT TGA TCA TTT TTA CAC GAC GGT -3' & $S f i \mathrm{I}$ \\
\hline OZG21 & 5'- ggccatctaggcc CCT GTT CAC CTC ACA ACC AAA TGG -3' & $S f i \mathrm{I}$ \\
\hline OZG22 & $5^{\prime}-\boldsymbol{g} \boldsymbol{t c g} \boldsymbol{a c}$ CGG CAC TTA CAG TAT CCT CCG GTA -3' & SalI \\
\hline OZG32 & 5'- CTT CAA AGG GCT CTC TGA GGT TGG CT -3' & \\
\hline OZG36 & $5^{\prime}$ - agatct TCA ATA CCC GCC AAT ATC TGC CTG -3' & $B g l \mathrm{II}$ \\
\hline OZG37 & $5^{\prime}-$ tctaga ATG GGT GCT CAG GTT ATC GCC GCT -3' & $X b a \mathrm{I}$ \\
\hline OZG47 & $5^{\prime}-\operatorname{ggg} \boldsymbol{g} c \boldsymbol{c}$ TCG TCG AAC CTA ATA AGT GGT AG -3' & ApaI \\
\hline OZG48 & $5^{\prime}-\operatorname{ggg} \boldsymbol{g} c \boldsymbol{c}$ GTC CCT TGA TAA ACG TTC CCT GG -3' & ApaI \\
\hline OZG49 & $5^{\prime}$ - actagt ATG GCT ACA CTT GCA GCA CCA CCA - $3^{\prime}$ & SpeI \\
\hline OZG50 & $5^{\prime}-\boldsymbol{c} \boldsymbol{c} \boldsymbol{c} \boldsymbol{c} \boldsymbol{t} \boldsymbol{g}$ TAA CGC ATG GTG GCA GGC TTT GAG A -3' & Pmi I \\
\hline
\end{tabular}




\section{Results}

\section{Neurospora crassa VELVET-1 displays high similarity to Aspergillus nidulans VeA}

The heterothallic ascomycete Neurospora crassa is known for its response to blue light, which is controlled by the protein WC-1. In the homothallic ascomycete Aspergillus nidulans, however, a developmental response to red light is more pronounced: illumination with red light inhibits sexual development and induces asexual conidiation through phytochromemediated signal transduction (Blumenstein et al., 2005), which presumably involves the veA gene product. The established roles of VeA in A. nidulans development raised the question as to whether there is any veA-like gene in $N$. crassa and what roles it may perform in the N. crassa light response.

Inspection of the $N$. crassa protein database (www.broad.mit.edu/annotation/ genome/neurospora/Home.html) disclosed one protein orthologous to A. nidulans VeA encoded by the locus NCU01731.2. According to the automatic annotation data, the locus contains two exons interrupted by one intron located in the first half of the gene with the capacity to encode a protein of 555 amino acids. This deduced protein displays a high degree of similarity to VeA of $A$. nidulans (similarity index of $49.5 \%$ ) and other VeA-like proteins of various Aspergillus species (Fig. 11A). Accordingly, the identified gene of N. crassa was designated ve-1. When aligning VE-1 with VeA by the EMBOSS Pairwise Alignment Algorithm (http://www.ebi.ac.uk/emboss/align/index.html), many conserved amino acid substitutions are noticable. VeA contains a putative nuclear localization signal (NLS) sequence in its N-terminus (Kim et al., 2002; Stinnett et al., 2007) and a putative nuclear export signal (NES,_http://www.cbs.dtu.dk/services/NetNES/) in the middle of the protein sequence, while VE-1 apparently does not comprise any classical NLS or NES motif. VeA also contains a so-named PEST (Pro, Glu or Asp Ser, Thr-rich) box responsible for protein degradation (Rechsteiner, 1990; Kim et al., 2002); when submitting the VE-1 sequence to the PEST finder internet tool (https://emb1.bcc.univie.ac.at/toolbox/pestfind/pestfind-analysiswebtool.htm), one putative PEST motif ( $\mathrm{K}^{172}$ DPEEPNAPPDGSPGSFDF $^{191}$ ) could be identified for this protein. Moreover, highly conserved patterns in the N-termini of both proteins exist, which might indicate a specific function of the $\mathrm{N}$-terminus. This is supported by the $A$. nidulans veAl allele, which encodes an N-terminal-truncated protein and results in reduced levels of sexual development (Mooney et al., 1990). Transcription factor map alignment based on the upstream regions of both genes demonstrate that conserved stretches along the 5' untranslated regions (5' UTRs) can be detected (Fig. 11B), and suggests similar elements conferring transcriptional regulation of both genes. 
A

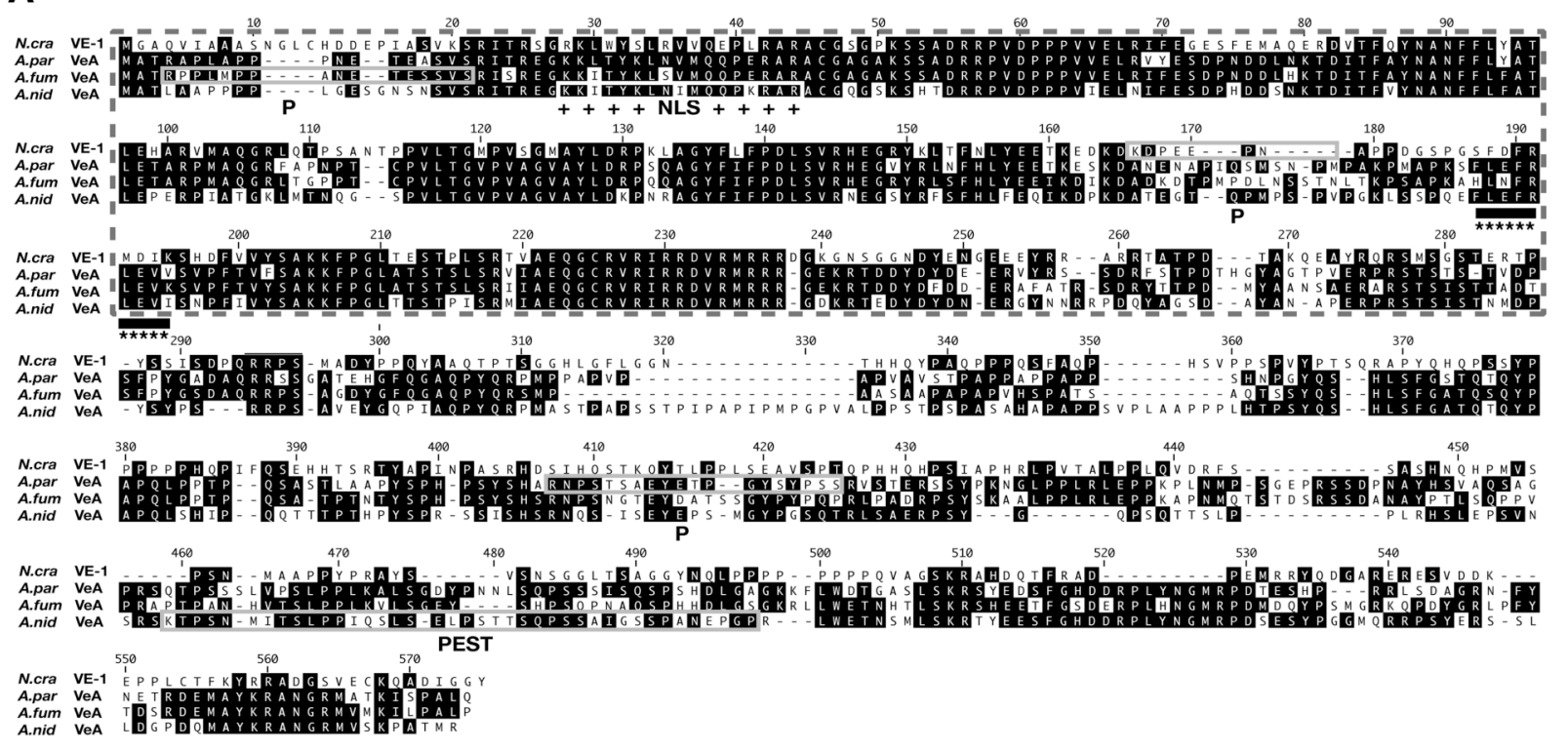

B

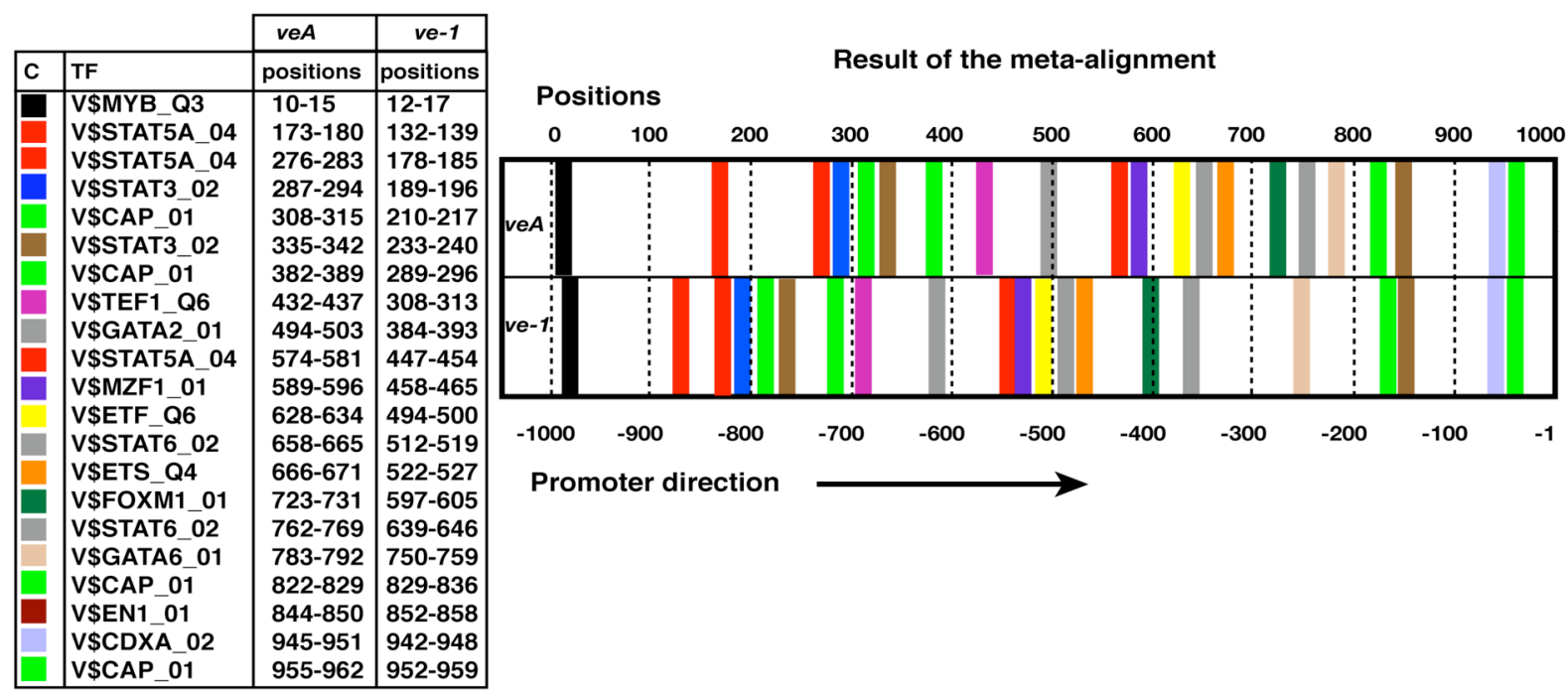

Figure 11: Velvet proteins are evolutionary conserved in filamentous fungi. (A) Clustal $\mathrm{W}$ alignment of velvet proteins from Aspergillus species and Neurospora Crassa with conserved residues shaded in black. The proteins comprise several highly conserved motifs in their N-termini, shown as large gray-dashed rectangle. A. nidulans VeA bears a bipartite nuclear localization signal (NLS) motif (white rectangle and plus symbol), and all VeA orthologues contain a putative PEST (Pro, Ser, Asp, Thr) motif in different positions of the proteins (gray rectangles). A. nidulans VeA also bears a potential nuclear export signal (NES) indicated as thick black bar and stars. N. cra: N. crassa VE-1; A. par: A. parasiticus VeA; A. fum: A. fumigatus VeA; A. nid: A. nidulans VeA. (B) Result of TF-map alignment (Blanco et al., 2006) considering 1000 nucleotides upstream of the veA and $v e-1$ coding sequence, respectively. TF-maps were created using TRANSFAC matrices at a threshold of 0.75 to result in 190 elements for the veA and 149 elements for the ve-1 upstream region. The deduced TF-map alignment, containing 21 elements, is schematically presented on the right hand side. Transcription factors with coordinates of respective binding sites are listed on the left hand side table (C: colour, TF: transcription factor designation). 


\section{Deletion of ve-1 results in increased conidiation associated with stunted aerial hyphae}

Observing the high degree of conservation of the $N$. crassa ve-1 gene product, it was tempting to address the cellular function of this protein in order to answer the question if it has conserved functions in N. crassa. For that aim, we constructed a deletion module containing a hygromycin resistance cassette flanked by $2.5 \mathrm{~kb}$ upstream and downstream regions of ve-1. The construct was transformed into the recipient $N$. crassa strain FGSC987 (74-OR23-1A), and a homologous gene replacement event was screened by Southern hybridization using a probe complementary to the 5'UTR region of ve-1 (Fig. 12A, B). As a result, three transformants could be confirmed to carry the desired gene replacement.

These deletion strains were backcrossed with the wild-type strain in order to remove secondary genetic defects and were screened for their normal developmental stages. All three deletion strains were identical in phenotypic appearance, therefore one representative, strain NCOB1, was investigated further. This ve-1 $\Delta$ deletion strain displays various phenotypic defects: an increase in asexual sporulation associated with reduced aerial hyphae formation on Vogel's minimal medium (Fig. 12C, D). In addition, its hyphal branching pattern appears slightly disturbed (not shown). The deletion strain's growth rate is reduced by $15 \%$, which is probably due to the hyphal branching disturbance. The increase in conidiation was quantified by counting the spores produced in slants to be almost a three fold more than the FGSC987 (74-OR23-1A) (Fig. 12D). All phenotypes, aerial hyphae and asexual development abnormalities, could be complemented by introducing a $7 \mathrm{~kb}$ genomic fragment comprising the ve-1 gene locus. The deletion strain was also investigated for its ability for sexual propagation, to reveal that it is both female- and male-fertile producing ascospore-containing perithecia on cornmeal agar to the same extent as the wild-type $N$. crassa progenitor strain. One additional phenotype, which is blocked protoperithecia development on synthetic crossing (SC) media, could, however, not be complemented by the $7 \mathrm{~kb}$ genomic fragment (data not shown).

The fact that the mutant is able to form protoperithecia on cornmeal medium but not on the defined synthetic one is elusive but may be based on the complex nature of the former growth substrate. The deletion strain did not reveal any abnormality under different light sources. These findings suggest that VE-1 is important for the regulation of hyphal growth, morphology and repression of asexual sporulation in $N$. crassa, but differences in sexual development on two tested media may imply the existence of some redundantly acting gene products during sexual development in N. crassa. 
A
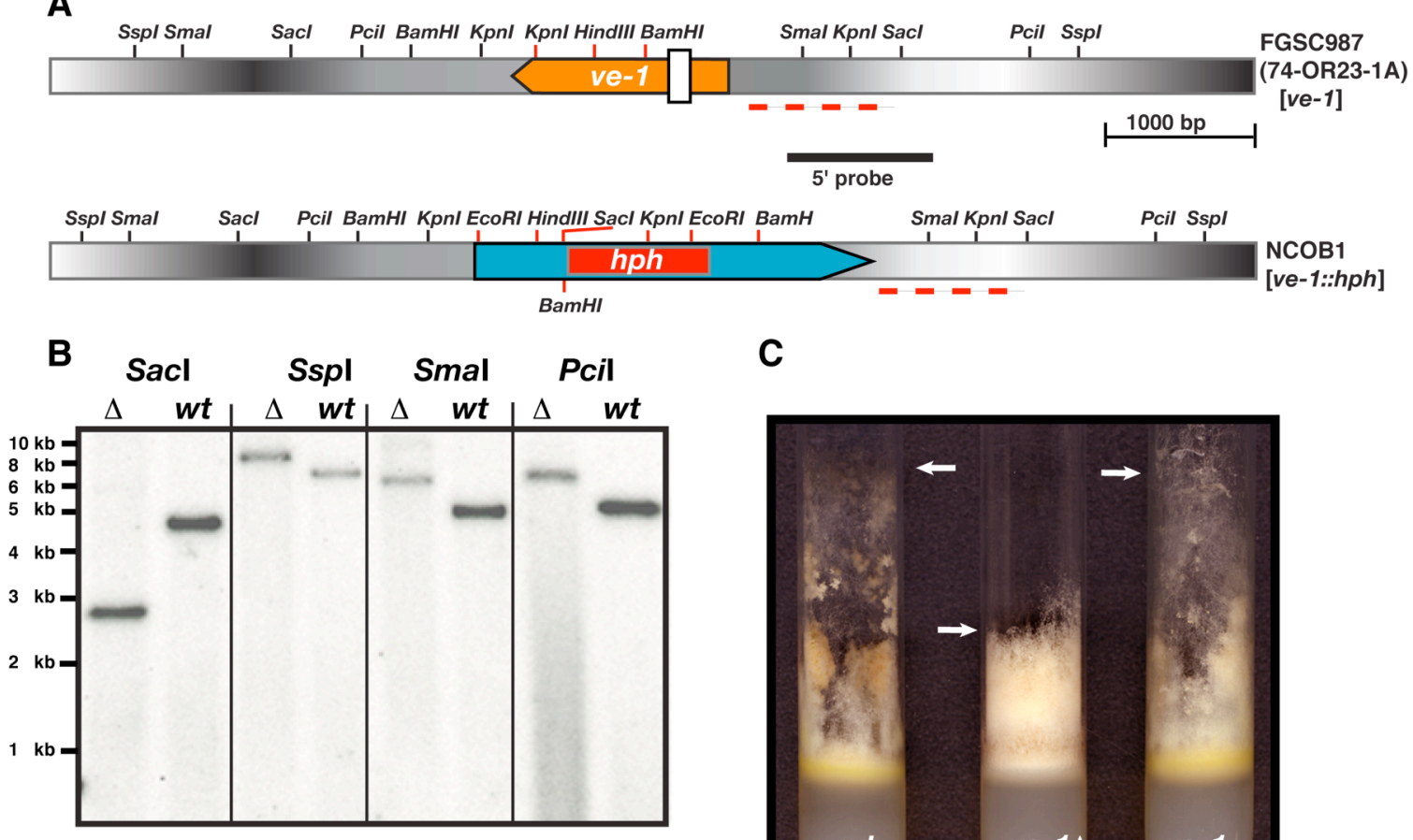

C

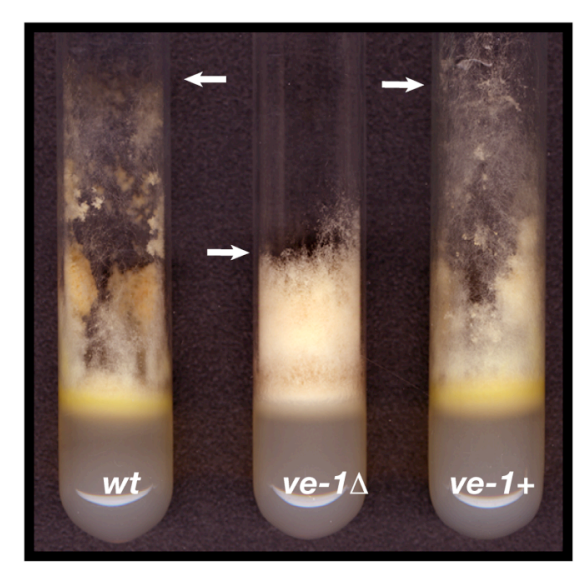

D

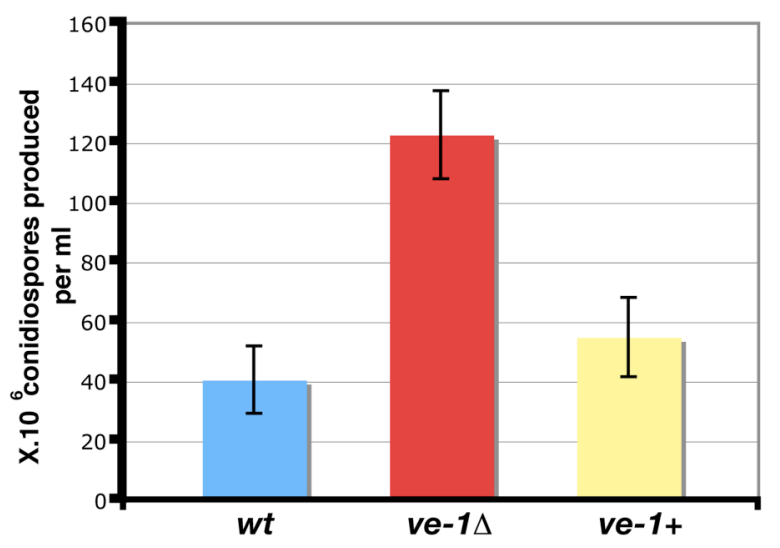

Figure 12: Deletion of ve-1 results in increased conidiation associated with stunted aerial hyphae formation. (A) Comparative depiction of the wild-type ve-1 locus in strain FGSC987 (74-OR23-1A) and the ve$1 \Delta:: h p h$ locus in the knock-out strain NCOB1. The black bar indicates the region encompassed by the probe employed in Southern analyses. (B) Autoradiography results of Southern hybridization validates the homologous gene replacement [sizes of detected restriction fragments as deduced from gene loci maps: SacI: 2.9 kb for deletant ( $(\Delta), 4.9 \mathrm{~kb}$ for wild-type (wt); SspI: $9.1(\Delta)$ vs. $7.5 \mathrm{~kb}(\mathrm{wt}) ;$ SmaI: $6.8(\Delta)$ vs. $5.2 \mathrm{~kb}(\mathrm{wt}) ;$ PciI: $7.2(\Delta)$ vs. $5.6 \mathrm{~kb}(\mathrm{wt})$; sizes of length standard fragments were extrapolated from the initial agarose gel electrophoresis image]. Phenotypical investigation of the $N$. crassa ve-1 $1 \Delta$ strain unveils shortened aerial hypha formation (C) and increased asexual conidiospore formation (D); both defects are complemented by re-transformation with the $v e-1$ genomic locus. White arrows in (C) indicate the edge of upward growing hyphae. Strains were grown at room temperature for five days, conidiospores were quantified by counting spores from different four tubes for each strain; standard deviations are indicated as black bars. 


\section{$v e-1$ is transcribed constitutively during different life stages of $N$. crassa}

Temporal and spatial expression of conserved regulators determine the developmental fate of related organisms. The expression of $v e A$ in A. nidulans is regulated during the course of distinct developmental stages: during vegetative growth it is transcribed at basal levels, whereas transcript levels intensify during asexual development and become stronger for the period of sexual development (Kim et al., 2002).

Accordingly, we considered that there might be a similar regulation of ve- 1 expression in $N$. crassa. Mycelia of the vegetative growth phase was grown in Vogel's minimal media for $15 \mathrm{~h}$ under constant shaking. Asexual development was triggered in Vogel's minimal cultures grown for five days without shaking to induce conidiation, and sexual development was induced by mycelial growth for three days in liquid SC medium followed by incubation on SC plates for an additional five days. Northern hybridisation experiments with RNA preparations from these different developmental stages revealed that, in contrast to veA expression in $A$. nidulans, ve-1 appears to have a constitutive transcription pattern throughout the probed developmental stages of $N$. crassa (Fig. 13A), indicating a general importance of the ve-1 gene during all life phases of the organism and not only during one specific stage.

A

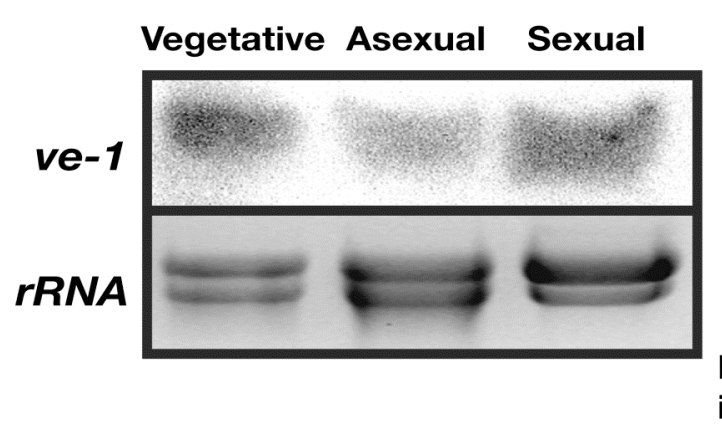

B

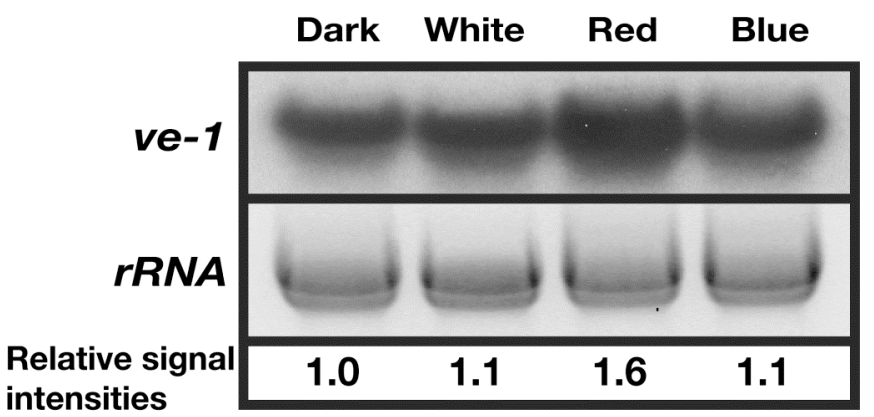

Figure 13: ve-1 expression is constitutive during development and increases upon red light illumination.

(A) Northern hybridization of ve-1 displays constant expression levels during vegetative growth (15 h, in Vogel's liquid minimal media culture), asexual (five days in Vogel liquid minimal media without shaking) and sexual development on SC (three days in synthetic crossing liquid media, and subsequently plated on SC plates for additional five days). (B) For light-dependent expression analysis, ve-1 expression was monitored under illumination by different light sources in Vogel's minimal media grown at $30{ }^{\circ} \mathrm{C}$ for two days. Northern hybridisation intensities of ve-1 under red light conditions are elevated by $60-70 \%$, as quantified from autoradiographies obtained from two independent experimental setups.

A. nidulans and N. crassa exhibit red and blue light responses, respectively. Hence we asked whether ve-1 expression is regulated by different wavelengths emitted from light sources. Cultures of $N$. crassa were grown without shaking at $30^{\circ} \mathrm{C}$ for 2 days under white, blue, and red light illumination, respectively, or in the darkness and analyzed for the 
expression of ve-1 by Northern blot hybridisation (Fig. 13B). Inspection of the resulting autoradiographies revealed that, when compared to dark and white light conditions, steadystate transcript levels of ve- 1 increase under red light, indicating a light-dependent regulation of $v e-1$ expression.

\section{VE-1 and the ve-1 promoter are functional in $A$. nidulans}

To test if the two velvet proteins are functionally conserved among these distantly related filamentous ascomycetes, we expressed the $N$. crassa protein VE-1 in A. nidulans to test whether it is a functional orthologue of $\mathrm{VeA}$, and to monitor its regulation in the heterologous host. For that purpose, we constructed a set of chimeric expression constructs. One plasmid (pME3179) contains the ve- 1 coding sequence driven by the $A$. nidulans veA promoter and terminator sequences to test functionality of the ve- 1 coding sequence in A. nidulans. This construct was transformed into the veA $\Delta$ deletion strain DVAR1 to yield AGB285. While a wild-type A. nidulans strain develops cleistothecia after four to five days at $37^{\circ} \mathrm{C}, \mathrm{AGB} 285$ needed about 8-9 days under identical incubation conditions to produce fruiting bodies that bear meiotically-derived, fertile ascospores (Fig. 14). In addition to this delayed complementation of the sexual development defect, a brown pigmentation, which is a prominent phenotype caused by the veA $\Delta$ deletion, was absent (Fig. 14).

After determining that ve- 1 expressed from the veA promoter is functional, we tested if $v e A$ expression in $A$. nidulans can be accomplished by the ve-1 promoter. For that purpose, we devised a chimeric plasmid (pME3180) containing a veA cDNA under the control of the $N$. crassa ve-1 promoter and terminator sequences and transformed it into the A. nidulans

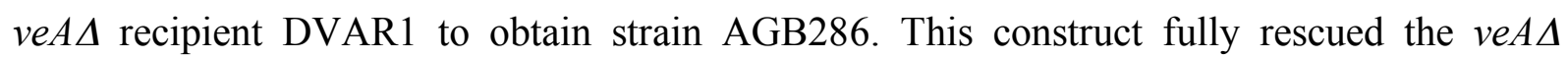
deletion phenotypes, which are characterized by the absence of fruiting bodies and excessive brown pigmentation (Fig. 14).

Finally, we tested whether a native genomic DNA fragment of ve- 1 is also functional in A. nidulans. A PCR-amplified fragment of the ve- 1 genomic locus comprising $1.6 \mathrm{~kb}$ of 5 , and 3' UTRs was transformed into the veAS strain DVAR1, yielding AGB287, and was found to fully restore the deletion phenotypes to result in ascospore-containing fruiting bodies and to reconstitute the brownish pigmentation phenotype (Fig. 14). In all cases, the cleistothecia formed by any complementation construct were filled with fertile ascospores (data not shown). 


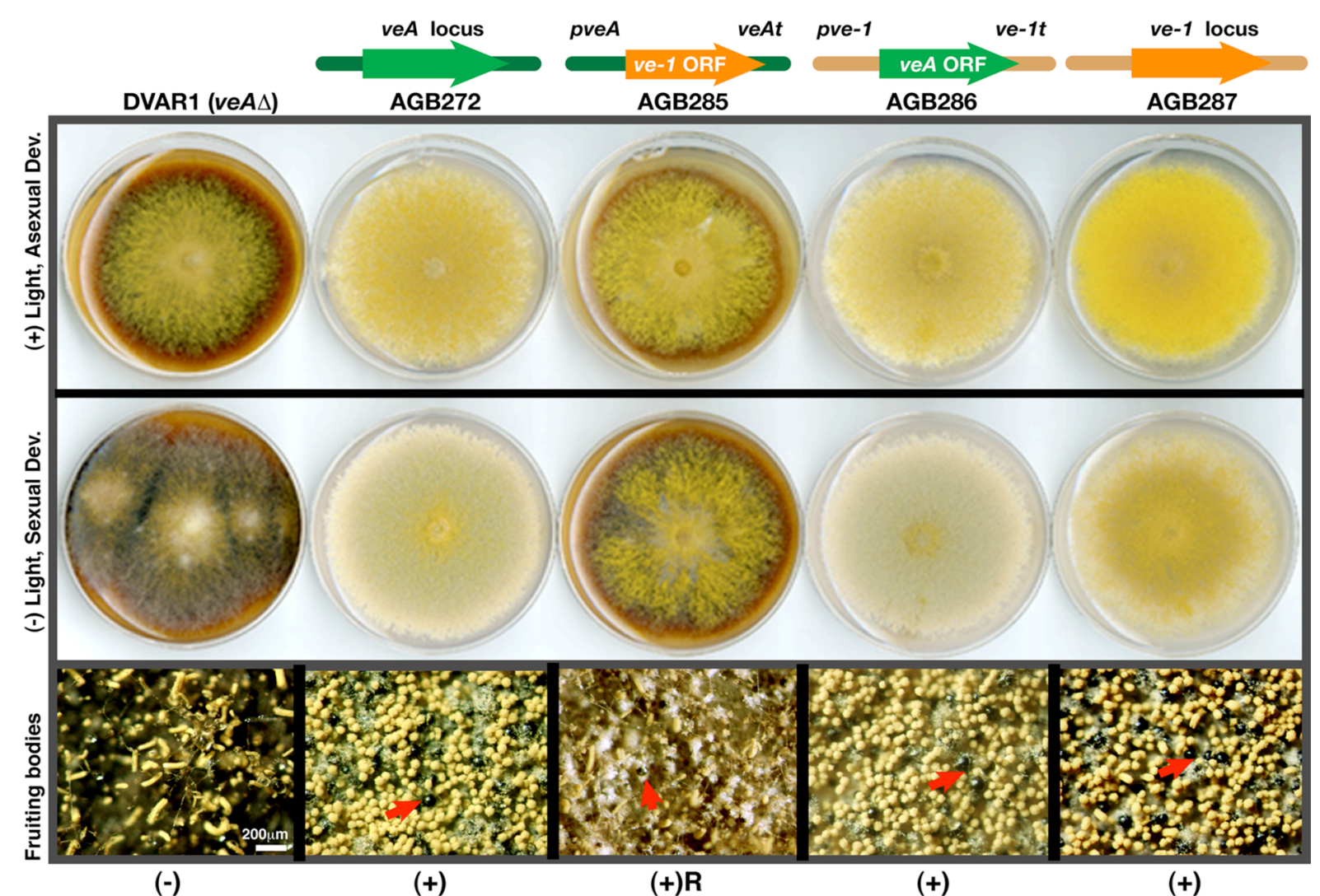

Figure 14: The $N$. crassa velvet gene is a functional orthologue of $\boldsymbol{A}$. nidulans veA. $A$. nidulans strain DVAR1 (veAA), which is impaired in fruiting body formation and defective in asexual sporulation associated with brown pigment production, was transformed with velvet genes from A. nidulans or $N$. crassa. As control, a native genomic fragment of veA was used that entirely rescued the veA null phenotype of DVAR1 (AGB272). 10 ${ }^{3}$ conidia were inoculated per plate and incubated at $37^{\circ} \mathrm{C}$ for 3 days in the presence of light for asexual development and for 5 days in the darkness to propagate sexual development, respectively. In strain AGB285, the veA deletion phenotype is partially complemented as brown pigmentation is reduced and the strain is able to form fruiting bodies with a delay of 4-5 days. pME3180 as expressed in AGB286 behaves identical to the native A. nidulans fragment. The $N$. crassa ve-1 genomic fragment of $5 \mathrm{~kb}$ length fully complements all defects of DVAR1 (AGB287). The potograph of the AGB285 fruiting bodies was taken on day 8 due to strains delayed development, red arrows indicate mature cleistothecia.

We also quantified the number of conidiospores and fruiting bodies produced by the strains expressing the chimeric genes in comparison to the veA $\Delta$ progenitor DVAR1. Strains bearing the veA native genomic locus (AGB272) and the ve-1 genomic locus (AGB287) produced comparable amounts of conidiospores while in the strains that express chimeric constructs (AGB285 and AGB286), the defect in conidiospore production is only partially rescued (Fig. 15A). Furthermore, the amount of fruiting bodies generated in the chimeric veAbearing strain (AGB286) is comparable to the wild-type situation of AGB272 (Fig. 15B), while cleistothecia formation in AGB287 (ve-1 genomic fragment) is slightly lower. The low 
numbers of cleistothecia produced in AGB285 $\left({ }^{p} v e A: \because v e-1: \because v e A^{t}\right)$ might be a result of interference of the 5 ' intron that lies within the veA promoter with the intron in the ve-1 coding sequence. Taken together, all three constructs complement the characteristic phenotypes of an $A$. nidulans veA $\Delta$ mutant strain, validating the functional conservation of the velvet factor between homothallic and heterothallic ascomycetous fungi. In N. crassa, the function of VE-1 in influencing asexual sporulation negatively is more obvious, but our data indicate a role of $v e A$ also in $A$. nidulans' asexual development. Functionality of the $N$. crassa ve- 1 coding sequence and its promoter elements in $A$. nidulans, which also mirrors the lightdependent regulation of expression, suggests that either the protein is regulated in the same manner or conserved promoter sequences are responsive to the light regulation in A. nidulans. The latter notion may be substantiated by the fact that the 5' UTRs of both orthologous genes contain conserved regions.

A

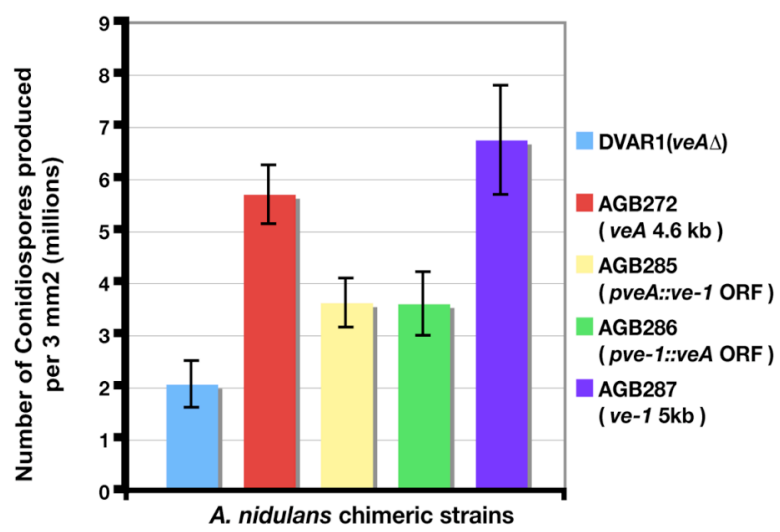

B

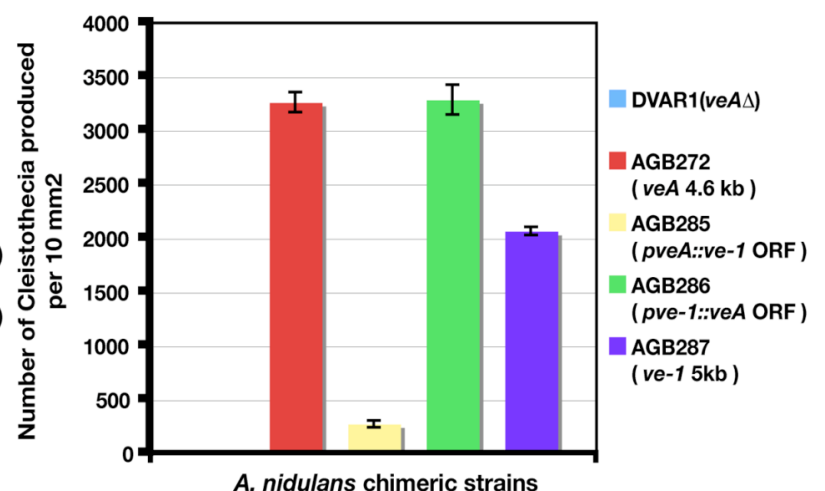

Figure 15: Quantitative depiction of the cross-complementations studies on $N$. crassa ve-1 constructs expressed in A. nidulans. (A) Asexual spore productions of chimeric strains per $3 \mathrm{~mm}^{2}$ : the DVAR1 strain produces reduced numbers of conidia, which is reconstituted by an A. nidulans genomic fragment comprising veA (AGB272) as well as by the chimeric constructs as expressed in strains AGB285, AGB286, and AGB287. (B) Cleistothecia produced per $10 \mathrm{~mm}^{2}:$ A nidulans veAs strain (DVAR1) is defective in cleistothecia formation, and fruiting bodies' production is restored in strains AGB272 and AGB286. AGB287 also displays complementation of the $v e A \Delta$ phenotype, however forming slightly less fruiting bodies than AGB272. Strain AGB285 forms less fruiting bodies with 4-5 days retardation. For each strain different sectors from three independent plates were counted, standard deviations are indicated. 


\section{Discussion}

The recent sequencing efforts and publications on fungal genomes have revealed many orthologous and paralogous genes conserved throughout this kingdom. By detailed inspection of genes responsible for different metabolic, developmental, and morphological pathways functional or structural conservations could be uncovered. One interesting fungal protein is the so-called velvet factor, which is unique for filamentous fungi. By analysis of the $N$. crassa genome, we disclosed one homologue to the velvet-encoding A. nidulans veA gene, and a sequence alignment of its deduced gene product VE-1 with VeA revealed a high degree of conservation. A. nidulans has an additional velvet-like protein designated as VelB, which has been identified as an interaction partner of $\mathrm{VeA}$ (our unpublished data); in silico analysis indicates that there is also a velB orthologue encoded by the $N$. crassa genome (NCU02775.3), suggesting conservation for this regulatory complex among ascomycetes.

A. nidulans $\mathrm{VeA}$ is required for the balance between asexual and sexual development (Kim et al., 2002). It is presumably inactivated by white or red light to stimulate sexual development in the darkness while it reduces asexual sporulation under these conditions (Champe et al., 1981; Mooney et al., 1990; Mooney and Yager, 1990; Timberlake, 1990). Red light regulation was elucidated partially by the characterization of an $A$. nidulans fphA $\Delta$ mutant that forms fruiting bodies inappropriately under red light illumination (Blumenstein et al., 2005). N. crassa, as a heterothallic ascomycete, displays two major life cycles: asexual conidiation and sexual development, and especially the latter is directed by internal and external factors. $N$. crassa fruiting bodies are produced independently of illumination. However, the perithecial ostiole is directed towards the source of light (Harding and Melles, 1983), and under blue light conditions, the number of protoperithecia is increased (Degli-Innocenti and Russo, 1984). In comparison, the A. nidulans illumination response is more pronounced with respect to red light, and there are no data describing any obvious UV or blue light response for this homothallic fungus.

Many genes that have been demonstrated to be involved in meiosis and ascus development are closely related in filamentous ascomycetes (Raju, 1992). In a sequence alignment, a striking conservation of the N-termini of the VeA and VE-1 proteins is evident (Fig. 1A), and there is functional evidence that this part of VeA is important for its regulatory function in $A$. nidulans development as many laboratory strains of $A$. nidulans carry a point mutation in the veA start codon which results in a truncated gene product (Kim et al., 2002). Strains expressing this $v e A 1$ allele produce decreased numbers of fruiting bodies and are not affected in their sporulation by red light illumination (Mooney and Yager, 1990). To address 
any particular functional conservation of the $\mathrm{N}$-termini, phenotypes resulting from expression of a correspondingly truncated $N$. crassa VE-1 protein might be assessed in future studies.

Our cross-complementation experiments demonstrate that VE-1, even when expressed from the heterologous $v e A$ promoter, is able to complement the phenotypes that derive from the loss of the veA gene in $A$. nidulans. The ve- 1 coding sequence under the control of the $A$. nidulans veA 5' UTR rescued the veA $\Delta$ phenotype in a slightly delayed manner. Moreover, the veA coding sequence could be functionally expressed from the ve-1 5' UTR, so the elements present in the promoter region of ve- 1 seem to be recognized by the transcriptional machinery of A. nidulans to result in proper expression of veA. Sequence alignments of the ve-1 and veA 5' UTRs provide evidence for conserved regions, which may serve as cis-acting elements. Although ve-1 expression in N. crassa is induced upon red light illumination, the function of VE-1 is seemingly not influenced by environmental illumination conditions. A. nidulans' red light response is mediated by the phytochrome FphA (Blumenstein et al., 2005), which was found to interact with VeA (Purschwitz et al., 2006). The N. crassa genome contains two phytochrome-like genes, that encode putative VE-1 interactors, and deletion of them results in no obvious phenotype (Froehlich et al., 2005). Deletion of fphA in A. nidulans, however, enhances fruiting body formation under red light illumination, which normally represses sexual development (Blumenstein et al., 2005). The presence of this red lightdependent phenotype in an A. nidulans but not an $N$. crassa phytochrome mutant might be an explanation why ve-1 gene does not act in a light-dependent fashion in N. crassa, while it is apparently regulated in a light-dependent manner in A. nidulans. Accordingly, it is likely that VE-1 incorporates into the same signal transduction pathway in A. nidulans.

Deletion of ve-1 in $N$. crassa caused the formation of shortened aerial hyphae accompanied by increased conidial production and a decreased tip extension with abnormal branching. One additional, but more enigmatic phenotype is the lack of protoperithecia formation in SC medium, a phenotype that could not be complemented in any of the analysed knock-out mutant strains. Despite the fact that this sexual phenotype cannot be complemented by reintroduction of the otherwise complementing ve- 1 genomic fragment, it might retain some hints for an elusive role of VE-1 during the sexual development of N. crassa. Redundancy with other factors such as the homologues of velB might obscure its function in protoperithecia formation. The pronounced aerial hypae phenotype seen in the ve- $1 \Delta$ strain is similar to that observed in an $A$. nidulans veA $\Delta$ strain during sexual development in darkness. However, aerial mycelia formation of a $v e A \Delta$ strain is not detectable during conidiation in the light. More interestingly, with the loss of ve-1, asexual sporulation is upregulated or 
derepressed in $N$. crassa, and the mutant forms more conidia than the wild-type strain FGSC987 (74-OR23-1A). This suggests that in N. crassa ve-1 represses asexual sporulation either in the light or in the darkness, whereas veA represses asexual conidiation of $A$. nidulans in the darkness. In addition, it is also required for asexual sporulation in the light, since a $v e A \Delta$ deletant is also defective in light-induced asexual conidiation (Kim et al., 2002). In $N$. crassa, ve- 1 overexpression from the $N$. crassa cpc- 1 promoter does not cause any abnormal phenotype (data not shown). In contrast, forced expression of veA in $A$. nidulans results in promiscuous production of sexual structures irrespectively of environmental conditions (Kim et al., 2002). Conclusively, N. crassa VELVET seems to be primarily a repressor of asexual conidiation, whereas $A$. nidulans velvet acts both as a repressor of asexual sporulation and as activator of fruiting body formation. Since the cross-complementation experiments confirm the functionality of VE-1 in A. nidulans, they are likely to operate similarly, and the observed difference could be due to the different downstream elements. Taken together, VE-1 has retained some repressor function for asexual conidiation, but somehow lost its sexual role to another factor in N. crassa.

Despite their structural conservation among filamentous ascomycetes, velvet orthologues seem to have gained different roles depending on the organism's need, expanding their conserved role in developmental programs. They can be adapted to the fungal-specific life cycle and may be involved in diverse functions such as sclerotia formation and toxin production like in A. parasiticus (Calvo et al., 2004), nutrition dependent sporulation like in A. fumigatus (Krappmann et al., 2005), or the microconidia-to-macroconidia ratio and cell wall formation like in the heterothallic fungus Fusarium verticilloides ( $\mathrm{Li}$ et al., 2006). Understanding the mechanisms of such diverse velvet-driven roles among filamentous fungi will require more work at the level of protein-protein interactions which holds the promise to elucidate the importance and the exact regulatory mechanism of these structurally and functionally conserved fungal-specific proteins. 


\section{References}

Adams, T.H., Boylan, M.T., and Timberlake, W.E. (1988). brlA is necessary and sufficient to direct conidiophore development in Aspergillus nidulans. Cell 54, 353-362.

Adams, T.H., Wieser, J.K., and Yu, J.H. (1998). Asexual sporulation in Aspergillus nidulans. Microbiol Mol Biol Rev 62, 35-54.

Andrianopoulos, A., and Timberlake, W.E. (1994). The Aspergillus nidulans abaA gene encodes a transcriptional activator that acts as a genetic switch to control development. Mol Cell Biol 14, 2503-2515.

Ballario, P., Vittorioso, P., Magrelli, A., Talora, C., Cabibbo, A., and Macino, G. (1996). White collar-1, a central regulator of blue light responses in Neurospora, is a zinc finger protein. Embo J 15, 1650-1657.

Bell-Pedersen, D., Cassone, V.M., Earnest, D.J., Golden, S.S., Hardin, P.E., Thomas, T.L., and Zoran, M.J. (2005). Circadian rhythms from multiple oscillators: lessons from diverse organisms. Nat Rev Genet 6, 544-556.

Blumenstein, A., Vienken, K., Tasler, R., Purschwitz, J., Veith, D., Frankenberg-Dinkel, N., and Fischer, R. (2005). The Aspergillus nidulans phytochrome FphA represses sexual development in red light. Curr Biol 15, 1833-1838.

Braus, G.H., Krappmann, S., and Eckert, S. (2002). Sexual development in ascomycetes: Fruit body formation in Aspergillus nidulans. In Molecular Biology of Fungal Development, Osiewacz, ed (New York: Marcel Dekker Inc), pp. 215-244.

Brown, T., and Mackey, K. (1997). Analysis of RNA by Northern and Slot Blot Hybridization. In Current Protocols in Molecular Biology (John Wiley \& Sons Inc.), pp. 4.9.1-4.9.16.

Calvo, A.M., Bok, J., Brooks, W., and Keller, N.P. (2004). veA is required for toxin and sclerotial production in Aspergillus parasiticus. Appl Environ Microbiol 70, 47334739. 
Cerda-Olmedo, E. (2001). Phycomyces and the biology of light and color. FEMS Microbiol Rev 25, 503-512.

Champe, S.P., Kurtz, M.B., Yager, L.N., Butnick, N.J., and Axelrod, D.E. (1981). Spore formation in Aspergillus nidulans: competence and other developmental processes. In Fungal spores: Morphogenic Controls, H.R. Hohl and G. Turian, eds (New York: Academic Press), pp. 255-276.

Crosson, S., Rajagopal, S., and Moffat, K. (2003). The LOV domain family: photoresponsive signaling modules coupled to diverse output domains. Biochemistry 42, 2-10.

Davis, R.D., and DeSerres, F.J. (1970). Genetic and microbiological research techniques for Neurospora crassa. Methods Enzymol. 17, 79-143.

Degli-Innocenti, F., and Russo, V.E. (1984). Isolation of new white collar mutants of Neurospora crassa and studies on their behavior in the blue light-induced formation of protoperithecia. J Bacteriol 159, 757-761.

Feinberg, A.P., and Vogelstein, B. (1983). A technique for radiolabeling DNA restriction endonuclease fragments to high specific activity. Anal Biochem 132, 6-13.

Froehlich, A.C., Liu, Y., Loros, J.J., and Dunlap, J.C. (2002). White Collar-1, a circadian blue light photoreceptor, binding to the frequency promoter. Science 297, 815-819.

Froehlich, A.C., Noh, B., Vierstra, R.D., Loros, J., and Dunlap, J.C. (2005). Genetic and molecular analysis of phytochromes from the filamentous fungus Neurospora crassa. Eukaryot Cell 4, 2140-2152.

Galagan, J.E., Calvo, S.E., Cuomo, C., Ma, L.J., Wortman, J.R., Batzoglou, S., Lee, S.I., Basturkmen, M., Spevak, C.C., Clutterbuck, J., Kapitonov, V., Jurka, J., Scazzocchio, C., Farman, M., Butler, J., Purcell, S., Harris, S., Braus, G.H., Draht, O., Busch, S., D'Enfert, C., Bouchier, C., Goldman, G.H., Bell-Pedersen, D., Griffiths-Jones, S., Doonan, J.H., Yu, J., Vienken, K., Pain, A., Freitag, M., Selker, E.U., Archer, D.B., Penalva, M.A., Oakley, B.R., Momany, M., Tanaka, T., Kumagai, T., Asai, K., Machida, M., Nierman, W.C., Denning, D.W., Caddick, M., Hynes, M., Paoletti, M., Fischer, R., Miller, B., Dyer, P., Sachs, 
M.S., Osmani, S.A., and Birren, B.W. (2005). Sequencing of Aspergillus nidulans and comparative analysis with A. fumigatus and A. oryzae. Nature 438, 1105-1115.

Galagan, J.E., Calvo, S.E., Borkovich, K.A., Selker, E.U., Read, N.D., Jaffe, D., FitzHugh, W., Ma, L.J., Smirnov, S., Purcell, S., Rehman, B., Elkins, T., Engels, R., Wang, S., Nielsen, C.B., Butler, J., Endrizzi, M., Qui, D., Ianakiev, P., BellPedersen, D., Nelson, M.A., Werner-Washburne, M., Selitrennikoff, C.P., Kinsey, J.A., Braun, E.L., Zelter, A., Schulte, U., Kothe, G.O., Jedd, G., Mewes, W., Staben, C., Marcotte, E., Greenberg, D., Roy, A., Foley, K., Naylor, J., StangeThomann, N., Barrett, R., Gnerre, S., Kamal, M., Kamvysselis, M., Mauceli, E., Bielke, C., Rudd, S., Frishman, D., Krystofova, S., Rasmussen, C., Metzenberg, R.L., Perkins, D.D., Kroken, S., Cogoni, C., Macino, G., Catcheside, D., Li, W., Pratt, R.J., Osmani, S.A., DeSouza, C.P., Glass, L., Orbach, M.J., Berglund, J.A., Voelker, R., Yarden, O., Plamann, M., Seiler, S., Dunlap, J., Radford, A., Aramayo, R., Natvig, D.O., Alex, L.A., Mannhaupt, G., Ebbole, D.J., Freitag, M., Paulsen, I., Sachs, M.S., Lander, E.S., Nusbaum, C., and Birren, B. (2003). The genome sequence of the filamentous fungus Neurospora crassa. Nature 422, 859-868.

Gyula, P., Schafer, E., and Nagy, F. (2003). Light perception and signalling in higher plants. Curr Opin Plant Biol 6, 446-452.

Hanahan, D., Jessee, J., and Bloom, F.R. (1991). Plasmid transformation of Escherichia coli and other bacteria. Methods Enzymol 204, 63-113.

Harding, R.W., and Melles, S. (1983). Genetic Analysis of Phototropism of Neurospora crassa Perithecial Beaks Using White Collar and Albino Mutants. Plant Physiol 72, 996-1000.

He, Q., Cheng, P., Yang, Y., Wang, L., Gardner, K.H., and Liu, Y. (2002). White collar-1, a DNA binding transcription factor and a light sensor. Science 297, 840-843.

Idnurm, A., and Heitman, J. (2005). Light controls growth and development via a conserved pathway in the fungal kingdom. PLoS Biol 3, e95.

Käfer, E. (1965). Origins of translocations in Aspergillus nidulans. Genetics 52, 217-232. 
Kim, H., Han, K., Kim, K., Han, D., Jahng, K., and Chae, K. (2002). The veA gene activates sexual development in Aspergillus nidulans. Fungal Genet Biol 37, 72-80.

Kolar, M., Punt, P.J., van den Hondel, C.A., and Schwab, H. (1988). Transformation of Penicillium chrysogenum using dominant selection markers and expression of an Escherichia coli lacZ fusion gene. Gene 62, 127-134.

Krappmann, S., Bayram, O., and Braus, G.H. (2005). Deletion and allelic exchange of the Aspergillus fumigatus veA locus via a novel recyclable marker module. Eukaryot Cell 4, 1298-1307.

Kües, U. (2000). Life history and developmental processes in the basidiomycete Coprinus cinereus. Microbiol Mol Biol Rev 64, 316-353.

Li, S., Myung, K., Guse, D., Donkin, B., Proctor, R.H., Grayburn, W.S., and Calvo, A.M. (2006). FvVE1 regulates filamentous growth, the ratio of microconidia to macroconidia and cell wall formation in Fusarium verticillioides. Mol Microbiol 62, 1418-1432.

Linden, H., and Macino, G. (1997). White collar 2, a partner in blue-light signal transduction, controlling expression of light-regulated genes in Neurospora crassa. Embo J 16, 98-109.

Linden, H., Ballario, P., and Macino, G. (1997). Blue light regulation in Neurospora crassa. Fungal Genet Biol 22, 141-150.

Liu, Y., He, Q., and Cheng, P. (2003). Photoreception in Neurospora: a tale of two White Collar proteins. Cell Mol Life Sci 60, 2131-2138.

Maheshwari, R. (1999). Microconidia of Neurospora crassa. Fungal Genet Biol 26, 1-18.

Marshall, M.A., and Timberlake, W.E. (1991). Aspergillus nidulans wetA activates sporespecific gene expression. Mol Cell Biol 11, 55-62.

Mooney, J.L., and Yager, L.N. (1990). Light is required for conidiation in Aspergillus nidulans. Genes Dev 4, 1473-1482. 
Mooney, J.L., Hassett, D.E., and Yager, L.N. (1990). Genetic analysis of suppressors of the veA1 mutation in Aspergillus nidulans. Genetics 126, 869-874.

Pöggeler, S., Nowrousian, M., and Kück, U. (2006). Fruiting-Body Development in Ascomycetes. In The Mycota I Growth, Differentiation and Sexuality, K. Fischer, ed (Heidelberg: Springer-Verlag), pp. 325-355.

Punt, P.J., and van den Hondel, C.A. (1992). Transformation of filamentous fungi based on hygromycin B and phleomycin resistance markers. Methods Enzymol 216, 447-457.

Purschwitz, J., Muller, S., Kastner, C., and Fischer, R. (2006). Seeing the rainbow: light sensing in fungi. Curr Opin Microbiol 9, 566-571.

Raju, N.B. (1992). Genetic control of the sexual cycle in Neurospora. Mycol. Res. 96, 241262.

Rechsteiner, M. (1990). PEST sequences are signals for rapid intracellular proteolysis. Semin Cell Biol 1, 433-440.

Saiki, R.K., Bugawan, T.L., Horn, G.T., Mullis, K.B., and Erlich, H.A. (1986). Analysis of enzymatically amplified beta-globin and HLA-DQ alpha DNA with allele-specific oligonucleotide probes. Nature 324, 163-166.

Sambrook, J., Fritsch, E.F., and Maniatis, T. (1989). Molecular Cloning: A Laboratory Manual. (Cold Spring Harbor, NY: Cold Spring Harbor Laboratory Press).

Schwerdtfeger, C., and Linden, H. (2003). VIVID is a flavoprotein and serves as a fungal blue light photoreceptor for photoadaptation. EMBO J 22, 4846-4855.

Seiler, S., Vogt, N., Ziv, C., Gorovits, R., and Yarden, O. (2006). The STE20/Germinal Center Kinase POD6 Interacts with the NDR Kinase COT1 and Is Involved in Polar Tip Extension in Neurospora crassa. Mol Biol Cell 17, 4080-4092.

Siegel, R.W., Matsuyama, S.S., and Urey, J.C. (1968). Induced macroconidia formation in Neurospora crassa. Experientia 24, 1179-1181.

Sohn, K.T., and Yoon, K.S. (2002). Ultrastructural study on the cleistothecium development in Aspergillus nidulans. Mycobiology 30, 117-127. 
Southern, E.M. (1975). Detection of specific sequences among DNA fragments separated by gel electrophoresis. J Mol Biol 98, 503-517.

Springer, M.L. (1993). Genetic control of fungal differentiation: the three sporulation pathways of Neurospora crassa. Bioessays 15, 365-374.

Springer, M.L., and Yanofsky, C. (1989). A morphological and genetic analysis of conidiophore development in Neurospora crassa. Genes Dev 3, 559-571.

Stinnett, S.M., Espeso, E.A., Cobeno, L., Araujo-Bazan, L., and Calvo, A.M. (2007). Aspergillus nidulans VeA subcellular localization is dependent on the importin alpha carrier and on light. Mol Microbiol 63, 242-255.

Thompson, J.D., Higgins, D.G., and Gibson, T.J. (1994). CLUSTAL W: improving the sensitivity of progressive multiple sequence alignment through sequence weighting, position-specific gap penalties and weight matrix choice. Nucleic Acids Res 22, 46734680 .

Timberlake, W.E. (1990). Molecular genetics of Aspergillus development. Annu Rev Genet 24, 5-36.

Vollmer, S.J., and Yanofsky, C. (1986). Efficient cloning of genes of Neurospora crassa. Proc Natl Acad Sci U S A 83, 4869-4873.

Yager, L.N. (1992). Early developmental events during asexual and sexual sporulation in Aspergillus nidulans. Biotechnology 23, 19-41.

Zonneveld, B.J.M. (1977). Biochemistry and ultrastructure of sexual development in Aspergillus. (London: Academic Press). 


\title{
Chapter 4
}

\section{The velvet complex coordinates light, fungal development and secondary metabolism}

\begin{abstract}
Differentiation and secondary metabolism are correlated processes in fungi and respond to light. In Aspergillus nidulans, light inhibits sexual reproduction as well as secondary metabolism. We identify the heterotrimeric velvet complex as a link between light-responding developmental regulation and epigenetic control of secondary metabolism. VeA, which is primarily expressed in the dark, physically interacts with VelB that is expressed during sexual development. VeA bridges VelB stoichiometrically to the nuclear methyltransferase LaeA, an established master regulator of secondary metabolism. Deletion of either velB or veA results in defects in both sexual fruiting body formation and production of secondary metabolites.
\end{abstract}




\section{Introduction}

Secondary metabolites are small bioactive molecules including important biotechnological products such as antibiotics as well as potent toxins (Cole, 2003). The secondary metabolism is a common feature of plants, fungi and bacteria but is atypical for animals. Whereas the primary metabolism is essential for growth, the loss of the potential to produce the secondary metabolites does not result in the death of an organism. Secondary metabolic pathways are often tightly correlated with the developmental programmes and are triggered by external cues including light. Light is sensed by a plethora of organisms ranging from archaea to mammals with specific photosensing molecules. Light-driven processes not only include changes in secondary metabolites but also growth, morphogenesis or the adjustment of circadian rhythms (Gyula et al., 2003; Lin and Todo, 2005).

The mould A. nidulans forms air-borne asexual spores in light, but preferentially undergoes sexual reproduction in the dark (Zonneveld, 1977; Adams et al., 1998). This results in the formation of sexual fruit bodies called cleistothecia that consist of different cell types and in an increase in secondary metabolism (Braus et al., 2002b). Secondary metabolites of $A$. nidulans include 'friends and foes' of human health as the antibiotic penicillin (Brakhage, 1997) and the carcinogenic aflatoxin (AF) precursor sterigmatocystin (ST), respectively (Hicks, 2002). Mutations resulting in defects in fungal development often also impair secondary metabolism (Calvo et al., 2002). There is some genetic evidence for a connection between fruit body formation, secondary metabolism and light in A. nidulans, but the molecular mechanism is yet elusive (Kim et al., 2002; Busch et al., 2003; Kato et al., 2003).

The A. nidulans mutants lacking the red light receptor phytochrome gene $f p h A$ are partially blind and produce more fruit bodies under red light (Blumenstein et al., 2005). Whereas plant phytochromes translocate into the nucleus upon light exposure (Kircher et al., 2002), the A. nidulans phytochrome FphA is localized in the cytoplasm under all tested conditions (Blumenstein et al., 2005). This implies the presence of additional components that transmit the red light signal into the fungal nucleus. One candidate for such a bridge is the enigmatic and conserved velvet protein encoded by the veA gene whose expression is significantly increased during sexual development (Kim et al., 2002; Krappmann et al., 2005; Li et al., 2006). An interaction between VeA and the phytochrome FphA has been proposed (Purschwitz et al., 2006) and the transport of VeA into the nucleus is inhibited by light (Stinnett et al., 2007). VeA might act as a negative regulator of asexual development whose function is suppressed by light (Yager, 1992). 
Fungal secondary metabolite biosynthetic genes are often clustered and regulated by pathwayspecific transcription factors (Woloshuk et al., 1994; Keller et al., 2005). In addition, secondary metabolism is regulated at a higher hierarchic level by a global epigenetic control mechanism. The nuclear LaeA protein, which seems to be present in numerous fungi, has been identified as a master regulator of secondary metabolism in aspergilli (Bok and Keller, 2004). The deletion of laeA has a minute impact on morphological and developmental processes, but results in silencing of numerous secondary metabolite gene clusters including those responsible for the syntheses of the antibiotic penicillin as well as the toxins such as ST or gliotoxin (Keller et al., 2005; Bok et al., 2006). LaeA appears to be a nuclear methyltransferase, which is proposed to control the accessibility of chromatin regions that include secondary metabolite clusters (Bok et al., 2006). Although the lack of the developmental veA gene impairs ST and penicillin production (Kato et al., 2003), there is no experimental evidence for a direct or indirect interaction between the VeA developmental controller and the LaeA secondary metabolism regulator. 


\section{Materials and Methods}

\section{Strains, media, and growth conditions}

Fungal strains are listed in Table 7. A. nidulans TNO2A3 which displays a $v e A^{+}$phenotype served as wild-type for the velB deletion, AGB152 and DVAR1 were used for overexpression (Kim et al., 2002; Busch et al., 2003; Nayak et al., 2006). A velB replacement cassette comprising $2 \mathrm{~kb}$ of $v e l B$ upstream and downstream flanking regions and the pyrithiamine resistance gene ptrA as selection marker was created (Fig. 23A) and introduced into the $n k u A \Delta$ background strain TNO2A3 (Nayak et al., 2006); correct gene replacement was confirmed by Southern analyses (Fig. 23B). E. coli DH5 $\alpha$ and MACH-1 (INVITROGEN) were applied for plasmid DNA and were propagated in LB medium (1\% tryptone, $0.5 \%$ yeast extract, $1 \% \mathrm{NaCl}$ ) supplemented with $100-150 \mu \mathrm{g} \cdot \mathrm{ml}^{-1}$ ampicillin. The bacterial strain $\mathrm{KS} 272$ for recombinogenic engineering was propagated in low-salt $(0.5 \% \mathrm{NaCl}) \mathrm{LB}$ medium with 25 $\mu \mathrm{g} \cdot \mathrm{ml}^{-1}$ chloramphenicol. Minimal medium $\left(0.52 \mathrm{~g} \cdot \mathrm{l}^{-1} \mathrm{KCl}, 0.52 \mathrm{~g} \cdot \mathrm{l}^{-1} \mathrm{MgSO}_{4}, 1.52 \mathrm{~g} \cdot \mathrm{l}^{-1}\right.$ $\mathrm{KH}_{2} \mathrm{PO}_{4}, 0.1 \%$ trace element solution, $\mathrm{pH}$ 6.5) was used for growth of fungal strains, supplemented with appropriate amounts of 4-aminobenzoic acid (PABA, $1 \mu \mathrm{g} \cdot \mathrm{ml}^{-1}$ ), Biotin $\left(0.02 \mu \mathrm{g} \cdot \mathrm{ml}^{-1}\right)$, Uracil $\left(50 \mu \mathrm{g} \cdot \mathrm{ml}^{-1}\right)$, Pyridoxine $\left(0.05 \mu \mathrm{g} \cdot \mathrm{ml}^{-1}\right)$, nourseothricin-dihydrogen sulfate (100-120 $\left.\mu \mathrm{g} \cdot \mathrm{ml}^{-1}\right)$ (clonNAT, WERNER BIOAGENTS), pyrithiamine (TAKARA Bio Inc) $\left(0.1 \mu \mathrm{g} \cdot \mathrm{ml}^{-1}\right) ; 1 \%$ D-glucose was used as the source of carbon together with $10 \mathrm{mM}$ ammonium or nitrate as nitrogen source. For TAP* tag experiments, fungal strains were grown in complete medium (0.5\% yeast extract, $1 \%$ bacto-peptone, $1 \%$ glucose $)$. Sterigmatocystin production of strains was assayed as described (Bok and Keller, 2004).

\section{Transformation procedures}

E. coli and A. nidulans cells were transformed as described (Hanahan et al., 1991; Punt and van den Hondel, 1992).

\section{Plasmid constructions details}

The plasmids utilized in this work are listed in Table 8, oligonucleotide sequences are given in Table 9. As cloning plasmids, pBluescript II KS (StRATAGENE) and pUC19 (FERMENTAS) were used. The plasmid pME3156 containing veA::ctap* tag fusion was constructed by recombineering an $800 \mathrm{bp}$ EarI fragment comprising a C-terminal fusion of the TAP* tag to the veA coding sequence derived from pME3154 with NaeI-linearised pME3155 in E. coli. The recombineering is a genetic engineering based on homologous recombination in an $E$. 
coli host strain expressing phage-derived proteins (Muyrers et al., 2000). In order to create pME3157, a ptrA pyrithiamine resistance cassette were amplified with oligonucleotides Sv129/130 from pPTRII (TAKARA) and inserted into the SmaI site of pME3156, and the final construct was used in tandem affinity purification experiments. The veA::sgfp fusion in pME3168 was created by replacing the C-TAP* tag module in pME3154 by an OZG28/29amplified $s g f p$ fragment digested with NcoI/HindIII. pME3168 was digested with SmaI and a blunt ptrA (Sv129/130) was inserted resulting in pME3169. Gpd1/Nat2-amplified $1.4 \mathrm{~kb}$ ${ }^{p}$ gpdA::nat $^{R}$ cassette from pNV1 was cloned in SmaI site of pME3168 creating pME3167. To create a velB deletion construct, a $2 \mathrm{~kb}$ US flanking region was amplified (OZG57/58) and inserted into the EcoRV site of pBluescript KS II (STRATAGENE); the resulting plasmid was then used for insertion of a $2 \mathrm{~kb}$ velB DS flanking region (OZG59/60) into SmaI site, which was then digested with $S f i \mathrm{I}$ to insert the SfiI-released ptrA marker from pME3024 (Krappmann et al., 2006) generating pME3158, from which a $5.9 \mathrm{~kb}$ replacement cassette was used for deletion of velB locus. For complementation, pME3159 was created by cloning a 5 kb velB genomic fragment (OZG99/100) in the ApaI site of pNV1 (Seiler et al., 2006). For overexpression and localization experiments, the nitrogen source-dependent expression module of pME3160 was exploited, which contains the A. nidulans niiA/niiD intergenic region flanked by the corresponding termination regions to allow expression of two genes in a bidirectional orientation at the same time. The veA and velB cDNAs were amplified and cloned in PmeI site of pME3160 yielding pME3161 veA and pME3162 velB overexpression constructs, respectively. The velB cDNA::sgfp fusion construct was created by a fusion PCR (Yu et al., 2004) with OZG63/116 for velB and OZG115/29 for sgfp. To create a dominant

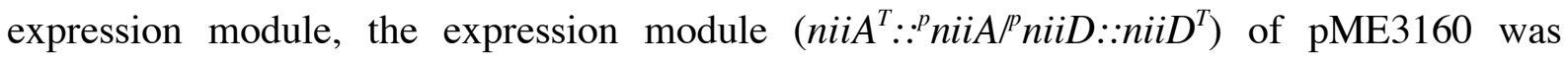
amplified with Sv315/318 and cloned into ApaI-digested flushed pNV1 to yield pME3166. The velB cDNA and velB cDNA::sgfp recombinant DNA fragments were cloned into the PmeI site of pME3166. To obtain the ${ }^{p}$ gpdA::mrfp ::h2A construct, the gpdA promoter and intron (Sv337/338), mrfp (Sv339/340), h2A cDNA containing terminator (Sv339/340) were amplified. Final products were fused using double joint PCR procedure (Yu et al., 2004). The ${ }^{p}$ gpdA::mrfp ::h2A recombinant fragment was cloned into the EcoRV site of pBluescript KS II followed by ${ }^{p} g p d A:: n a t^{R}$ cassette insertion into SmaI site yielding pME3173. pME3178 was constructed by subcloning HindIII genomic fragment of veA gene into HindIII site of pBluescript KS II followed by insertion of ptrA cassette into NotI site, which was finally transformed to DVAR1 yielding the complementation strain AGB272. Yeast two-hybrid bait constructs were formed by amplification of cDNAs from the sexual transcripts-specific cDNA 
library pCNS4 (Krappmann et al., 2006) and in-frame insertion in pEG202 (veA cDNA,

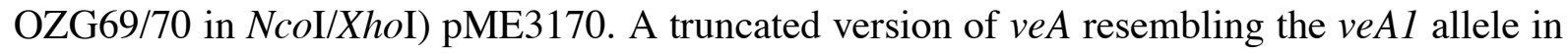
pEG202 (OZG98/Sv153 EcoRI) pME3171, the prey plasmid pJG 4-5 containing a velB cDNA (OZG67/68) and laeA cDNA (OZG65/66) in EcoRI/XhoI sites are designated as pME3172 and pME3189, respectively. For in vivo interaction experiments, n-eyfp (OZG73/74), veA cDNA (Sv142/143, same as OZG69/70 without restriction sites) and c-eyfp (OZG75/76), laeA cDNA (OZG61/62) were amplified and fused. The n-eyfp::veA and $c$ eyfp::laeA fusion constructs were cloned into the PmeI and SwaI sites of the pME3160, respectively. For the construction of the laeA::sgfp fusion plasmid (pME3190), laeA cDNA (OZG61/162) and sgfp (OZG29/161) were amplified, fused and inserted into the PmeI site of pME3166 expression module under the niiA promoter.

\section{Yeast two-hybrid analysis}

pEG202-derived bait and pJG4-5-derived prey vectors were co-transformed into S. cerevisiae strain EGY48-p1840 and transformants were selected on selective media SC-3 (-his, -trp, ura) containing leucine and 2\% glucose (Gyuris et al., 1993; Golemis and Brent, 1996). To assess leucine prototrophy, an appropriate volume of overnight grown cells was diluted and spotted on SC-4 (-his, -leu, -trp, -ura) plates containing 2\% galactose/ 1\% raffinose and lacking leucine to take pictures on the fourth day of growth. $\beta$-galactosidase assays were performed with cells grown for three days on membrane filters on SC-3 galactose/raffinose plates supplemented with leucine. Following shock-freezing in liquid nitrogen, membrane filters were soaked with $2.5 \mathrm{ml} \mathrm{Z}$-buffer $\left(60 \mathrm{mM} \mathrm{Na}_{2} \mathrm{HPO}_{4}, 40 \mathrm{mM} \mathrm{NaH}_{2} \mathrm{PO}_{4}, 10 \mathrm{mM} \mathrm{KCl}\right.$, $1 \mathrm{mM} \mathrm{MgSO}_{4}$, pH 7.0 ) comprising $60 \mu \mathrm{l}$ of $10 \% \mathrm{X}$-gal solution and were incubated at $30{ }^{\circ} \mathrm{C}$ for $3 \mathrm{~h}$. The blue colouring caused by $\beta$-galactosidase was documented at the end of the incubation period.

\section{Recombinant DNA procedures, hybridisation techniques and analysis of nucleic acids}

For recombinant DNA technology, standard protocols were performed (Sambrook et al., 1989b; Chaveroche et al., 2000). Taq, Pfu (MBI FermentAs) and Platinum Taq DNA polymerase (INVITROGEN) were used in PCR reactions (Saiki et al., 1986), and the cloning steps were confirmed by sequencing. Fungal genomic DNAs were prepared from ground mycelia (Kolar et al., 1988), and Southern blot analyses were conducted as described (Southern, 1975). Total RNA samples were analysed by Northern hybridization as described (Brown and Mackey, 1997). The STRATAGENE Prime-It II kit was used to radioactively label hybridization probes in the presence of $\left[\alpha{ }^{32} \mathrm{P}\right]$ dATP (Feinberg and Vogelstein, 1983). To 
produce autoradiographs, washed membranes were exposed to KODAK X-Omat films. Sequence data were analysed using the LASERGENE software package from DNASTAR, and alignments were created by the Clustal W method (http://www.ebi.ac.uk/clustalw/index.html; (Thompson et al., 1994). PEST motifs were analysed on the web tool (https://emb1.bcc.univie.ac.at/toolbox/pestfind/pestfind-analysis-webtool.htm) and NES patterns were identified on the web tool (http://www.cbs.dtu.dk/services/NetNES/).

\section{TAP purification}

Fungal strains AGB272 and AGB273 grown in liquid culture were transferred onto CMM (minimal medium $+0.1 \%$ casein hydrolysate) plates, wrapped with parafilm, and covered with aluminium foil to induce sexual development. At $48 \mathrm{~h}$ post induction of sexual development, the differentiating mycelia were ground in liquid nitrogen to prepare crude extracts in $\mathrm{B}^{*}$ buffer (100 mM Tris- $\mathrm{HCl}$ pH7.6, $250 \mathrm{mM} \mathrm{NaCl}, 10 \%$ glycerol, 0.05\% NP-40, $1 \mathrm{mM}$ EDTA, $2 \mathrm{mM}$ DTT) supplemented with an EDTA-free protease inhibitors mix (ROCHE), phosphatase inhibitors (MERCK) and specified protease inhibitors as recommended in the procedure at the NCRR (http://depts.washington.edu/yeastrc/pages/ms tap1.html). Crude extracts were centrifuged for $20 \mathrm{~min}$ at $15000 \mathrm{~g}$ and transferred into $50 \mathrm{ml}$ falcon tubes. Protein extracts were incubated for $3 \mathrm{~h}$ on a rotator with $300 \mu \mathrm{l}$ of IgG sepharose 6 Fast Flow (AmERShAM) at $4{ }^{\circ} \mathrm{C}$. After that point, the standard protocol (Step 14) as outlined at the NCRR web site was followed with minor modifications. TEV cleavage was executed under

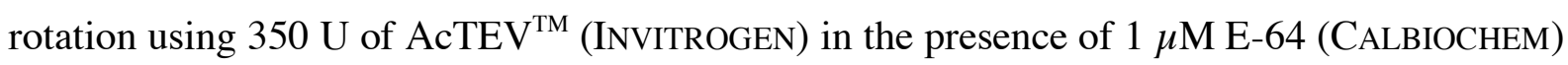
protease inhibitor for $5 \mathrm{~h}$ at $4^{\circ} \mathrm{C} ; 1 \mathrm{mM}$ PMSF (phenylmethanesulfonylfluoride) was included in the calmoduline binding step on an affinity resin (STRATAGENE). The TCA (trichloroacetic acid)-precipitated eluate was loaded onto a $10 \%$ polyacrylamide gel and stained with coomassie brilliant blue G (Sigma). Protein bands were cut out and submitted for mass spectrometry.

\section{Immunoblotting}

For the detection of the VeA::TAP* tag fusion protein and actin, anti-calmodulin binding peptide antibody (UPSTATE, catalog 07-482) and anti-actin antibody (MP Biomedicals, catalog 69100) were used respectively.

\section{Protein analysis by nano LC-MS}

Excised polyacrylamid gel pieces of protein bands were digested with trypsin as described by Shevchenko et al (Shevchenko et al., 1996). Peptides were then separated by water- 
acetonitrile gradients on Dionex-NAN75-15-03-C18-PM column with an ultimate-nanoHPLC system (Dionex, Bavel, the Netherlands). Online ESI-MS/MS2 spectra were generated on an LCQ-DecaXP ${ }^{\text {plus }}$ mass spectrometer (ThERmo FInNIGAN, San Jose, CA, USA). MS2 analysis and protein identification was done with the software SEQUEST/TURBOSEQUEST (Bioworks Browser 3.1, Thermo Finnigan) by using the Broad Institute public $A$. nidulans genome protein database (http://www.broad.mit.edu/annotation/genome/aspergillus nidulans/Home.html). VeA was identified by eight different peptides with SEQUEST (cross-correlation) XCorr values ranging from 5.27 to 2.11 , VelB by seven different peptides of XCorr values ranging from 5.18 to 2.72 , LaeA by three peptides (4.85 to 2.45 ), and KapA by four peptides (4.54 to 2.92).

\section{Fluorescence microscopy}

A. nidulans spores $\left(5.5 \times 10^{5}\right)$ were inoculated either on $18 \mathrm{~mm} \times 18 \mathrm{~mm}$ cover slips submerged in appropriately supplemented liquid medium or on large glass slides covered with a thin layer of medium and incubated at $30^{\circ} \mathrm{C}$ overnight. The effect of illumination on localization of VeA and VelB was investigated by growing selected strains in darkness and light (sometimes red light $680 \mathrm{~nm}$ ) on the agar surface or in the submerged culture. Cover slips were mounted on microscope slides using spore storage solution $(0.002 \%$ Tween, $0.5 \%$ $\mathrm{NaCl}$ ) and fixed with wax. Fluorescence photos were taken with a ZEISS Axiovert S100 microscope supported with a HAMAMATSU OCRA-ER digital camera, using the Openlab tmV5.0.1 software package (IMPROVISION, Coventry, UK). 
Table 7: Fungal strains used in this study

\section{Strain Genotype}

\section{Reference}

Aspergillus nidulans

\begin{tabular}{|c|c|c|}
\hline FGSC4 & Glasgow wild-type & FGSC \\
\hline FGSC26 & biAl, veAl & FGSC \\
\hline FGSC33 & biA1;pyroA4, veA1 & FGSC \\
\hline DVAR1 & pabaA1, yA2; $\arg B \Delta: \because \operatorname{trp} C ; \operatorname{trp} C 801 ; \operatorname{ve} A \Delta: \because \arg B$ & (Kim et al., 2002) \\
\hline \multirow[t]{2}{*}{ AGB272 } & \multicolumn{2}{|c|}{${ }^{p}$ veA::veA, ptrA; pabaA1, yA2; $\arg B \Delta: \because \operatorname{trp} C ; \operatorname{trp} C 801, v e A \Delta: \because \arg B$} \\
\hline & & This study \\
\hline \multirow[t]{2}{*}{ AGB273 } & \multicolumn{2}{|c|}{${ }^{p}$ veA::veA::ctap*tag, ptrA; pabaA1, yA2; $\arg B \Delta: \because \operatorname{trp} C ; \operatorname{trp} C 801$} \\
\hline & veA $A:: \arg B$ & This study \\
\hline \multirow[t]{2}{*}{ AGB274 } & \multicolumn{2}{|c|}{ 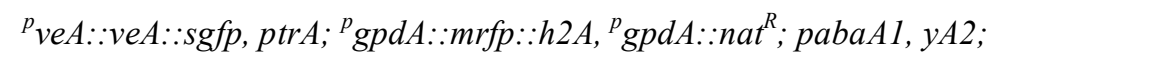 } \\
\hline & $\arg B \Delta:: \operatorname{trp} C ; \operatorname{trp} C 801, \operatorname{ve} A \Delta:: \arg B$ & This study \\
\hline \multirow[t]{2}{*}{ AGB275 } & \multicolumn{2}{|c|}{ 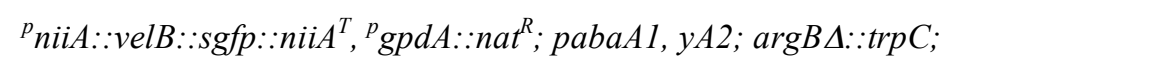 } \\
\hline & $\operatorname{trp} C 801, v e A \Delta:: \arg B$ & This study \\
\hline \multirow[t]{2}{*}{ AGB276 } & \multicolumn{2}{|c|}{${ }^{p}$ niiA::velB::niiA ${ }^{T},{ }^{p}$ gpdA::natR; pabaA1, yA2; $\arg B \Delta:: \operatorname{trp} C$} \\
\hline & $\operatorname{trp} C 801$, veA $\Delta:: \arg B$ & This study \\
\hline AGB152 & pyroA4, pyrG89, veA & (Busch et al., 2003) \\
\hline \multirow[t]{2}{*}{ AGB277 } & \multicolumn{2}{|c|}{ 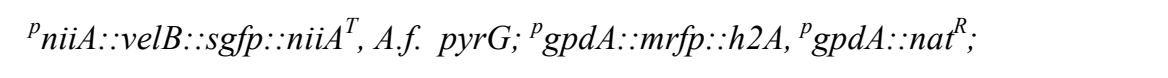 } \\
\hline & pyroA4, pyrG89 & This study \\
\hline AGB278 & ${ }^{p}$ niiA::velB::niiA ${ }^{T}$, A.f. pyrG; pyroA4, pyrG89 & This study \\
\hline TNO2A3 & pyrG89, pyro $A 4, \arg B 2 ; n k u A \Delta:: \arg B$ & (Nayak et al., 2006) \\
\hline AGB279 & velB $\Delta:: p \operatorname{tr} A ;$ pyrG89, pyro $A 4, \arg B 2 ; n k u A \Delta:: \arg B$ & This study \\
\hline \multirow[t]{2}{*}{ AGB280 } & \multicolumn{2}{|c|}{${ }^{p}$ velB::velB ${ }^{p}{ }^{g p d A}::$ nat ${ }^{R} ;$ velB $\Delta::$ ptrA; pyrG89, pyroA4, $\arg B 2 ;$} \\
\hline & $n k u A \Delta:: \arg B$ & This study \\
\hline \multirow[t]{2}{*}{ AGB281 } & \multicolumn{2}{|l|}{ 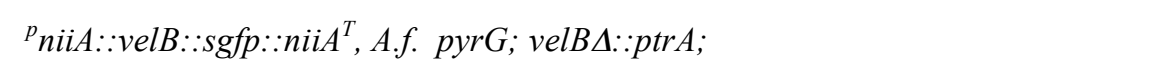 } \\
\hline & pyrG89, pyro $A 4, \arg B 2 ; n k u A \Delta:: \arg B$ & This study \\
\hline \multirow[t]{2}{*}{ AGB282 } & \multicolumn{2}{|c|}{${ }^{p}$ veA $:$ veA $:: \operatorname{sgfp},{ }^{p}$ gpdA::nat ${ }^{R} ;$ velB $\Delta:: p \operatorname{tr} A ;$ pyrG89, pyroA4, argB2; } \\
\hline & $n k u A \Delta:: \arg B$ & This study \\
\hline \multirow[t]{2}{*}{ AGB283 } & \multicolumn{2}{|c|}{${ }^{p}$ niiA $:$ veA $:: n i i A^{T}$, A.f. pyrG; velB $\Delta::$ ptrA; pyrG89, pyroA4, argB2; } \\
\hline & $n k u A \Delta:: \arg B$ & This study \\
\hline \multirow[t]{2}{*}{ AGB284 } & \multicolumn{2}{|c|}{${ }^{p}$ niiA::velB::niiA${ }^{T},{ }^{p}$ gpdA::nat ${ }^{R} ;$ pabaA1, yA2; $\arg B \Delta:: \operatorname{trp} C ; \operatorname{trp} C 801$} \\
\hline & veA $A:: \arg B$ & This study \\
\hline
\end{tabular}




\begin{tabular}{|c|c|c|}
\hline \multirow{2}{*}{ AGB307 } & \multicolumn{2}{|c|}{ 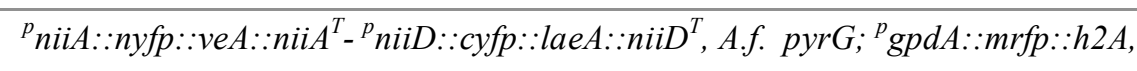 } \\
\hline & ${ }^{p}$ gpdA::nat ${ }^{R} ;$ pyroA4, pyrG89 & This study \\
\hline AGB308 & ${ }^{p}$ niiA $: \because l a e A: \because s g f p:: n i i A^{T},{ }^{p}$ gpdA $\because: n a t^{R}$ & This study \\
\hline \multirow[t]{2}{*}{ AGB310 } & \multicolumn{2}{|c|}{ 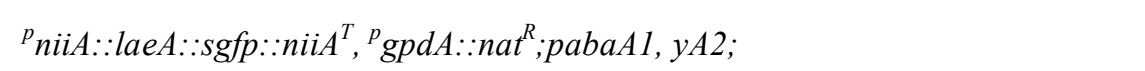 } \\
\hline & $\arg B \Delta:: \operatorname{trp} C ; \operatorname{trp} C 801 ; \operatorname{veA} \Delta:: \arg B$ & This study \\
\hline \multirow[t]{2}{*}{ AGB311 } & \multicolumn{2}{|c|}{ 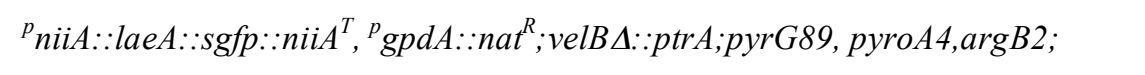 } \\
\hline & $n k u A \Delta:: \arg B$ & This study \\
\hline RNI 17.1 & velBA::Afpyr $G ; y A 2$ & This study \\
\hline RNI 17.2 & velBA::AfpyrG; yA2; pyroA4 & This study \\
\hline RNI 17.3 & velBA::AfpyrG & This study \\
\hline RNI 18.1 & velBA::AfpyrG; yA2 ; pyroA4; veA+ & This study \\
\hline RNI 18.2 & velBA::AfpyrG; yA2;veA+ & This study \\
\hline RNI 18.3 & velBA::AfpyrG; pyroA4; veA+ & This study \\
\hline \multicolumn{3}{|c|}{ Saccharomyces cerevisiae } \\
\hline \multicolumn{3}{|c|}{ EGY48-p1840 MATa his3 trp1 ura3-52 leu2::3LexAop-LEU2 LexAop-LacZ/URA3 } \\
\hline & & (Golemis and Brent, 1996) \\
\hline
\end{tabular}

FGSC: Fungal Genetics Stock Center, Kansas City, MO, USA. 
Table 8: Plasmid constructs employed in this study

\begin{tabular}{|c|c|c|}
\hline Plasmid & Description \& Characteristics & Reference \\
\hline pPTRII & autonomously replicating Aspergillus plasmid $[\mathrm{ptr} A$ & , AMA1, bla $]$ \\
\hline pEG202 & vector for the construction of LexA hybrid proteins & (Golemis and Brent, 1996) \\
\hline pJG4-5 & vector for the construction of B42 hybrid proteins & (Gyuris et al., 1993) \\
\hline pNV1 & Dominant resistance cloning plasmid & (Seiler et al., 2006) \\
\hline pME3024 & \multicolumn{2}{|c|}{ ptrA gene with $S f i$ sites in EcoRV site of pBlueII KS(Krappmann et al., 2006) } \\
\hline pME3154 & \multicolumn{2}{|c|}{ veA C-Terminus::ctap* tag::veA 3 ' UTR in pGEM5 This study } \\
\hline pME3155 & $v e A 4.6 \mathrm{~kb}$ HindIII genomic fragment in pUC19 & This study \\
\hline pME3156 & ${ }^{p} v e A: \because v e A: \because c$ tap $^{*}$ tag in $\mathrm{pUC} 19$ & This study \\
\hline pME3157 & ${ }^{p}$ veA::veA::ctap* tag, ptrA, in pUC19 & This study \\
\hline pME3158 & velB deletion cassette [velB::ptrA] & This study \\
\hline pME3159 & $5 \mathrm{~kb}$ velB locus amplicon in $A p a \mathrm{I}$ site of $\mathrm{pNV} 1$ & This study \\
\hline pME3160 & Exp. module niiA ${ }^{t}{ }_{-}^{p}$ niiA ${ }^{p}$ niiD-niiD ${ }^{t}-A f$ pyrG, bla & This study \\
\hline pME3161 & ${ }^{p}$ niiA $:$ veA cDNA in PmeI site of pME3160 & This study \\
\hline pME3162 & ${ }^{p}$ niiA $:$ velB cDNA in PmeI site of pME3160 & This study \\
\hline pME3163 & ${ }^{p}$ niiA::velB::sgfp in PmeI site of pME3160 & This study \\
\hline pME3164 & ${ }^{p}$ niiA::velB cDNA in PmeI site of pME3166 & This study \\
\hline pME3165 & ${ }^{p}$ niiA::velB::sgfp in PmeI site of pME3166 & This study \\
\hline \multirow[t]{2}{*}{ pME3166 } & \multicolumn{2}{|c|}{ Expression module $2.6 \mathrm{~kb}$ amplicon from pME3160 with primers Sv315/318 } \\
\hline & in ApaI site of pNV1 & This study \\
\hline pME3167 & ${ }^{p}$ veA::veA::sgfp, ${ }^{p}$ gpdA $:: n a t^{R}$ in pUC19 & This study \\
\hline pME3168 & ${ }^{p} v e A:: v e A:: s g f p$ in $\mathrm{pUC} 19$ & This study \\
\hline pME3169 & ${ }^{p} v e A:: v e A:: s g f p, p \operatorname{tr} A$ in $\mathrm{pUC} 19$ & This study \\
\hline pME3170 & $v e A$ cDNA in pEG202 YTH bait plasmid & This study \\
\hline pME3171 & $v e A 1 \mathrm{cDNA}$ in pEG202 YTH bait plasmid & This study \\
\hline pME3172 & velB cDNA in pJG4-5 YTH prey plasmid & This study \\
\hline \multirow[t]{2}{*}{ pME3173 } & \multicolumn{2}{|c|}{${ }^{p}$ gpdA::intron::mrfp::h2A cDNA in EcoRV and ${ }^{p} g p d A:: n a t^{R}$} \\
\hline & in SmaI of pBlueII KS & This study \\
\hline \multirow[t]{2}{*}{ pME3178 } & \multicolumn{2}{|l|}{ veA $4.6 \mathrm{~kb}$ HindIII genomic fragment in HindIII and ptrA } \\
\hline & in NotI site of pBlueII KS & This study \\
\hline \multirow[t]{2}{*}{ pME3188 } & \multicolumn{2}{|c|}{${ }^{p}$ niiA $:: n$-eyfp $:$ ve $A$ cDNA in pmeI, and ${ }^{p}$ niiD::c-eyfp $:: l a e A$ cDNA } \\
\hline & in $s w a$ I site of pME3160 expression module & This study \\
\hline pME3189 & laeA cDNA in pJG4-5 YTH bait plasmid & This study \\
\hline
\end{tabular}


Table 9: Oligonucleotides utilized for plasmid generations

\begin{tabular}{|c|c|c|}
\hline Designation & Sequence & Feature \\
\hline OZG28 & \multicolumn{2}{|c|}{ 5'-TTT GGC CAT GGG TGG TAG CGG TGG TAT GGT GAG CAA } \\
\hline & GGG CGA GGA GCT G-3' & $s g f p$-ggsgg-Spacer $(N c o \mathrm{I})$ \\
\hline \multirow[t]{2}{*}{ OZG29 } & \multicolumn{2}{|c|}{ 5'-AAA ATT TAA GCT TCT ACT TGT ACA GTT CGT CCA TGC } \\
\hline & CGT G-3' & $s g f p 3^{\prime}$ end (HindIII) \\
\hline OZG57 & 5'-ACT CAC GAA TCC ACG GGA TAC AT-3' & velB 5UTR-A \\
\hline \multirow[t]{2}{*}{ OZG58 } & \multicolumn{2}{|c|}{ 5'-GGC CTG AGT GGC CGG GTG GGA TAC GGT CCA TCG AAA-3' } \\
\hline & & velB 5UTR-B (sfiI) \\
\hline \multirow[t]{2}{*}{ OZG59 } & \multirow{2}{*}{\multicolumn{2}{|c|}{$\begin{array}{r}\text { 5'-GGC CAT CTA GGC CGA CCG TAT ATT GTT TCA TAA ATC CTT-3' } \\
\text { velB 3UTR-A (sfil) }\end{array}$}} \\
\hline & & \\
\hline OZG60 & 5'-TAT GAC CGC GTG AGC AAA TAG GAC-3' & velB 3UTR-B \\
\hline OZG61 & 5'-ATG TTT GAG ATG GGC CCG GTG GG-3' & laeA start \\
\hline OZG62 & 5'-TTA TCT TAA TGG TTT CCT AGC CTG GT-3' & laeA stop \\
\hline OZG63 & 5'-ATG TAC GCT GTT GAG GAT AGG GC-3' & velB start \\
\hline OZG64 & 5'-TTA GTA TTC GTT ATC CAG ACC ATC G-3' & velB stop \\
\hline OZG65 & \multicolumn{2}{|c|}{ 5'-GAA TTC ATG TTT GAG ATG GGC CCG GTG GG-3' laeA start (EcoRI) } \\
\hline OZG66 & \multicolumn{2}{|c|}{$5^{\prime}$-CTC GAG TTA TCT TAA TGG TTT CCT AGC CTG-3' laeA stop (XhoI) } \\
\hline OZG67 & \multicolumn{2}{|c|}{$5^{\prime}$-GAA TTC ATG TAC GCT GTT GAG GAT AGG GC-3'velB start (EcoRI) } \\
\hline OZG68 & \multicolumn{2}{|c|}{$5^{\prime}$-CTC GAG TTA GTA TTC GTT ATC CAG ACC ATC-3'velB stop (XhoI) } \\
\hline OZG69 & \multicolumn{2}{|c|}{$5^{\prime}$-CCA TGG ATG GCT ACA CTT GCA GCA CCA CC-3'veA start (NcoI) } \\
\hline \multirow[t]{2}{*}{ OZG70 } & \multicolumn{2}{|c|}{ 5'-CTC GAG TTA ACG CAT GGT GGC AGG CTT TGA GA-3' } \\
\hline & & veA sto \\
\hline OZG73 & 5'-ATG GTG AGC AAG GGC GAG GAG-3' & $n$-eyfp start \\
\hline \multirow[t]{2}{*}{ OZG74 } & \multicolumn{2}{|c|}{ 5'-GGT GGT GGT GCT GCA AGT GTA GCC ATC GTG GCG ATG GAG } \\
\hline & CGC ATG ATA TAG-3' & $n$-eyfp::veA fusion maker \\
\hline OZG75 & 5'-ATG GCC GAC AAG CAG AAG AAC-3' & c-eyfp start \\
\hline \multirow[t]{2}{*}{ OZG76 } & \multicolumn{2}{|c|}{ 5'-ACG AGT TCC CAC CGG GCC CAT CTC AAA CAT GTG GTT CAT } \\
\hline & GAC CTT CTG TTT CAG-3' & c-eyfp::laeA fusion maker \\
\hline \multirow[t]{2}{*}{ OZG98 } & \multicolumn{2}{|c|}{ 5'-TTT GAA TTC ATG CAG CAG CCC AAG CGC GCG AGA G-3' } \\
\hline & & veAl start \\
\hline OZG99 & $5^{\prime}$-AAA GGG CCC CGA GAA TGT CCG CCT G & $3^{\prime}$ velB complement-A (ApaI) \\
\hline OZG100 & 5'-CCA AGT CTG CCC GAC AAG CTC ACT G-3' & velB complement-B \\
\hline OZG115 & 5'-CGC CAC AGC GAC GAG GAC GAT GGT CT & AAC GAA TAC \\
\hline
\end{tabular}




\begin{tabular}{|c|c|}
\hline & GGT GGT AGC GGT GGT ATG GTG AGC AAG-3' \\
\hline OZG116 & 5'-GTA TTC GTT ATC CAG ACC ATC GTC-3' \\
\hline \multirow[t]{2}{*}{ OZG161 } & 5'-CTG CAC ATA TAC CAG GCT AGG AAA CCA TTA AGA GGT GGT \\
\hline & AGC GGT GGT ATG GTG AGC-3' \\
\hline OZG162 & 5'-TCT TAA TGG TTT CCT AGC CTG GTA-3' \\
\hline Sv129 & $5^{\prime}$-ATC TGA CAG AGC GGC CGC AAT TGA TTA CG-3' ptrA-A \\
\hline Sv130 & $5^{\prime}$-ATA TAT GCG GCC GCT CTT GCA TCT TTG TTT-3' ptrA-B \\
\hline Sv153 & $5^{\prime}$-TGA TCC CGC ATA TGA TCA GGG CGC CGG TT-3' veA primer for veAl \\
\hline Sv315 & 5'-GAT ACC AAA CGG AAC TGG CTG TTA TGG-3' \\
\hline Sv318 & 5'-ATC GAC GCA ACC ATC GAA GCA GC-3' \\
\hline Sv337 & 5'-GAT CTT TGC CCG GTG TAT GAA ACC-3' \\
\hline \multirow[t]{2}{*}{ Sv338 } & 5'-TCG GAG GAG GCC ATG GTG ATG TCT GCT CAA GC-3' \\
\hline & gpdA promoter $\mathrm{B}$ \\
\hline \multirow[t]{2}{*}{ Sv339 } & 5'-GAC ATC ACC ATG GCC TCC TCC GAG GAC GTC ATC-3' \\
\hline & $m r f p$ start \\
\hline \multirow[t]{2}{*}{ Sv340 } & 5'-GGC TCC AGC GCC TGC ACC AGC TCC GGC GCC GGT GGA GTG \\
\hline & GCG G-3' \\
\hline \multirow[t]{2}{*}{ Sv341 } & 5'-GGA GCT GGT GCA GGC GCT GGA GCC ACT GGC GGC AAA TCT \\
\hline & GGT G-3' \\
\hline Sv342 & 5'-ATC TGG AGG GGA CAG GCA GTT TAT-3' \\
\hline \multirow[t]{2}{*}{ GpdA } & 5'-GGG TTT CGA ACT ACA TCA AGG GTC CAA GAC CGA CAT CGA \\
\hline & GGC TCT GTA CAG TGACCG GTG-3' \\
\hline Nat2 & 5'-AGG GAA TTC TCA GGG GCA GGG CAT GC-3' \\
\hline
\end{tabular}




\section{Results}

\section{The A. nidulans velB gene product is a VeA-interacting protein}

The tandem affinity purification (TAP) protocol (Rohila et al., 2004) was adapted for A. nidulans and was employed to identify VeA interacting proteins (Fig. 16, 17). It is based on two tandemly repeated affinity tags. The first tag consists of two Staphylococcus aureus protein A domains (for IgG beads binding) that is separated from CBP (Calmodulin Binding Peptide) with a TEV protease cleavage site (Tobacco Etch $\underline{\text { Virus}) . ~ C B P ~ d o m a i n ~(f o r ~}$ Calmodulin affinity resin binding) is connected to the fusion protein through EKCS (Enterokinase $\underline{\text { Cleavage }} \underline{\text { Site) }}$ that is only present in the N-terminus TAP tag constructs. The advantage of TAP tag system is that it allows the purification of native protein complexes associated with the TAP tagged protein under mild salt conditions (Fig. 16).

A

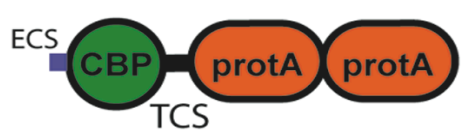

TAP tag

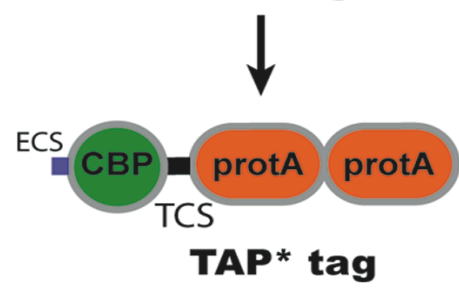

B

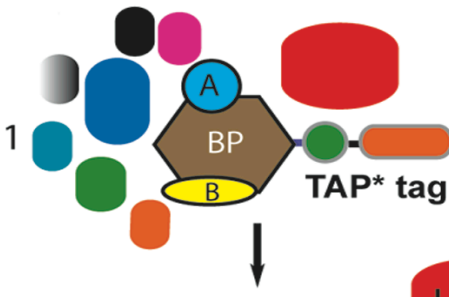

2
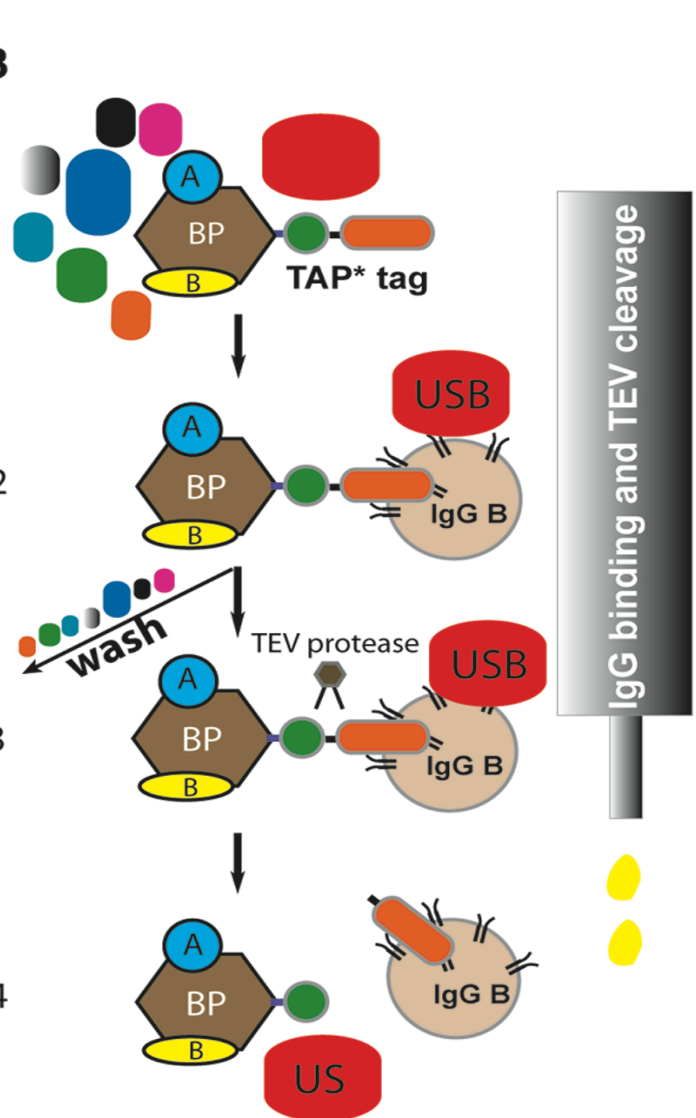
Figure 16: TAP (Tandem $\underline{\text { Affinity }}$ Purification) tag purificiation. (A) TAP tag contains two affinity tags: two tandemly repeated protein A epitopes which are connected to CBP (ㄷalmodulin $\underline{B}$ inding $\underline{\text { Peptide) }}$ through TEV (Tobacco Etch Virus) cleavage site. The N-terminal TAP tag contains an additional cleavage site, EKCS

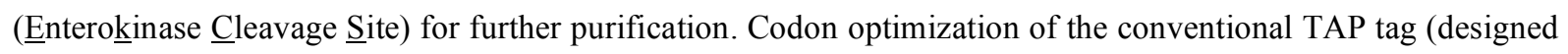
for yeast application) according to the $A$. nidulans codon usage without changing the feature of the tag (TAP* tag). (B) 1-2-3; the first purification steps based on the affinity between IgG antibodies covalently-bound to the beads and protein A epitope of the TAP tag. During the first purification, the tagged bait protein (BP) binds to the IgG beads with the proten A domain while the unspecific porteins flow through and unspecifically bound proteins are washed away except rare unspecific binding (USB). 3-4; The IgG-bound protein complex is cleaved by the site specific TEV protease and the cleaved complex containing CBP domain is incubated with calmodulin affinity resin in the presence of calcium ions (5-6) and washed with the same buffer and the pure protein

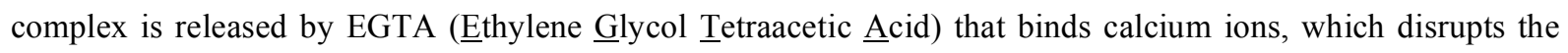
binding between resin and protein complex (7). Further purification when required, may be performed with Enterokinase cleavage or the elution fraction can be directly run in SDS gel and stained followed by the exicision of the bands, and submission to the mass spectrometry identification (8-9).

The strain expressing the functional veA::ctap* construct was further analysed. Protein enrichment was performed from dark-grown sexually induced cultures as well as from cultures that were kept in the light. Final eluates of the veA::ctap* A. nidulans strain and the $v e A$ wild-type control (wt) were visualised and analysed by mass spectrometry. Light-grown cultures resulted in low expression of VeA in comparison to significantly increased levels of VeA in the dark (Fig. 17).

A
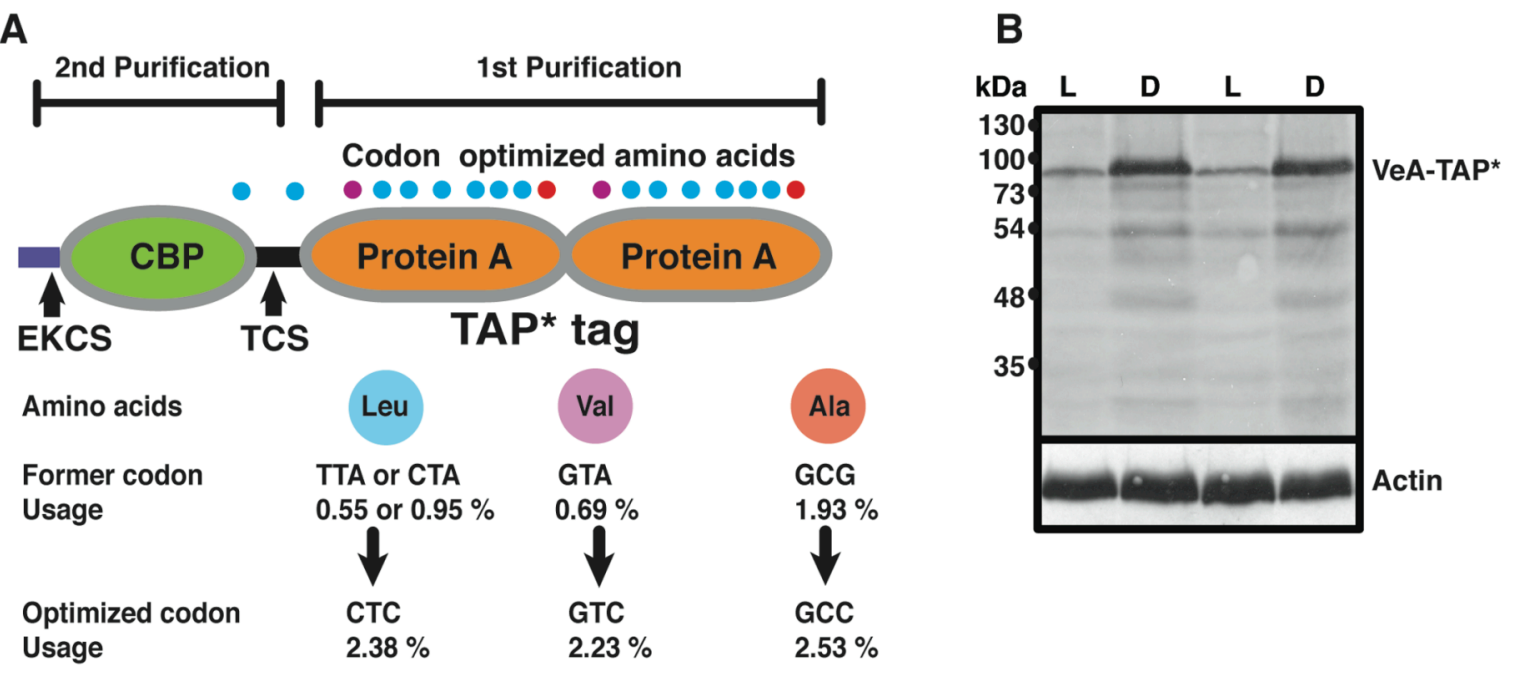

Figure 17: Codon modifications in the new TAP tag and expression of veA::c-tap* in the light and dark. (A) Depiction of the TAP tag. The codons for 18 amino acids were changed by a directed mutagenesis method (Kunkel, 1985) and are designated as differently coloured spheres. (B) Immunoblotting with antibody against calmodulin binding peptide: $85 \mathrm{kDa} \mathrm{VeA}: \mathrm{TAP} * \mathrm{tag}$, in the light (L) expression is relatively low in comparison to dark (D); as a control anti-actin antibody was used. 
Various proteins were co-purified with VeA::cTAP* in the dark (Fig. 18A and B). The protein in the first band was identified as VeA (AN1052.3). In bands 2 and 5, VeA and the AN0363.3 protein were present in a stoichiometric fashion. Based on the protein sequence similarity of AN0363.3 to VeA (Fig. 22), this newly identified protein was designated as VelB for $\underline{\boldsymbol{v}}$ lvet-like protein B. The low amounts of VeA-TAP tag fusion protein in lightgrown cultures also co-purified corresponding amounts of VelB (not shown). For further corroboration of the mass spectrometry data, yeast two-hybrid was performed cloning the VeA and VelB cDNAs into bait and prey plasmids, respectively. Yeast two-hybrid (Y2H) analysis (Gyuris et al., 1993) confirmed the interaction between VeA and VelB (Fig. 19, upper panel).

A

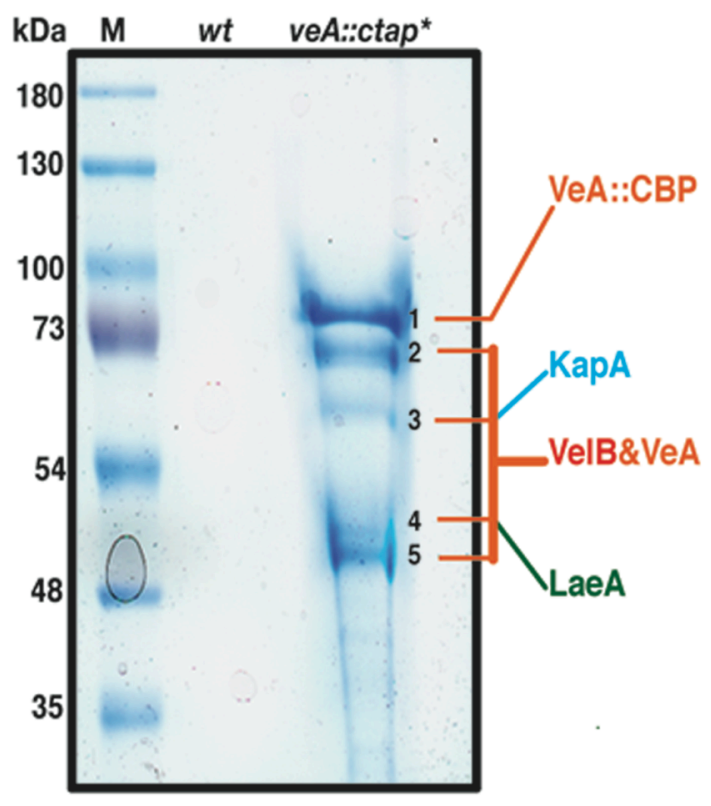

B

\begin{tabular}{|c|c|c|}
\hline Protein & Peptides identified & Bands \\
\hline $\begin{array}{c}\text { VeA } \\
\text { AN1052.3 }\end{array}$ & $\begin{array}{l}\text { LEVISNPFIVYSAK } \\
\text { AGYFIFPDLSVR } \\
\text { LSSPQEFLEFR } \\
\text { SSLLDGPDQMAYK } \\
\text { FPGLTTSTPISR } \\
\text { LNIMQQPK } \\
\text { LWETNSMLSK } \\
\text { KFPGLTTSTPISR }\end{array}$ & $\begin{array}{llll}1 & 2 & 3 & 45 \\
1 & 2 & \\
1 & 2 & 3 & 45 \\
1 & 2 & 3 & 455 \\
1 & 2 & 3 & 45 \\
1 & & \\
1 & & \\
& 2 & 345\end{array}$ \\
\hline $\begin{array}{c}\text { VelB } \\
\text { AN0363.3 }\end{array}$ & $\begin{array}{l}\text { IGVWFVLQDLSVR } \\
\text { IWSLQVVQQPIR } \\
\text { KFPGVIESTPLSK } \\
\text { NLIGCLSASAYR } \\
\text { FPGVIESTPLSK } \\
\text { RPITPPPCIR } \\
\text { DRRPITPPPCIR }\end{array}$ & $\begin{array}{r}45 \\
2345 \\
45 \\
45 \\
45 \\
2345 \\
4\end{array}$ \\
\hline $\begin{array}{c}\text { LaeA } \\
\text { AN0807.3 }\end{array}$ & $\begin{array}{l}\text { EIHAYNILHIYQAR } \\
\text { YAVAGGPAPWNR } \\
\text { HWYDCLK }\end{array}$ & $\begin{array}{l}4 \\
4 \\
4\end{array}$ \\
\hline $\begin{array}{c}\text { КарА } \\
\text { AN2142.3 }\end{array}$ & $\begin{array}{l}\text { IIQVALDGLENILK } \\
\text { TPQPDWNTIAPALPVLAK } \\
\text { IQAVIEAGIPR } \\
\text { GVFSDQIEAQIQATTK }\end{array}$ & $\begin{array}{l}3 \\
3 \\
3 \\
3\end{array}$ \\
\hline
\end{tabular}

Figure 18: Identification of VeA-associated proteins in A. nidulans. (A) Brilliant blue G-stained 10\% SDS PAGE gel of TAP procedure for VeA-associated proteins. One lane (wt, veA without tag) was loaded with the final eluate from strain AGB272, the other lane (veA::ctap*) was loaded with the eluate from AGB273. (B) The polypeptides identified from the bands belong to the corresponding proteins, lowest cross-correlation (XCorr) value for peptides was XCorr 2.11, M: molecular weight marker in kDa. 
The N-terminal part of VeA is required for the interaction with VelB which is expressed

\section{during late developmental stages}

VelB (Fig. 22) is conserved in the fungal kingdom and shares $18 \%$ amino acid identity with VeA. Whereas VelB has no typical nuclear localization signal (NLS) motif, VeA contains an NLS sequence in the N-terminus (Kim et al., 2002; Stinnett et al., 2007). The fungal $\alpha$ importin KapA is a VeA interacting protein (Fig. 18 A and B) which has been identified by $\mathrm{Y} 2 \mathrm{H}$; a truncated protein lacking the $\mathrm{N}$-terminal 37 amino acids (VeA1) is impaired in this interaction (Stinnett et al., 2007). The truncated VeA1 protein also showed impaired interaction with VelB, suggesting that the $\mathrm{N}$-terminus of $\mathrm{VeA}$ is required for interaction with both VelB and the $\alpha$ importin KapA (Fig. 19 upper panel).

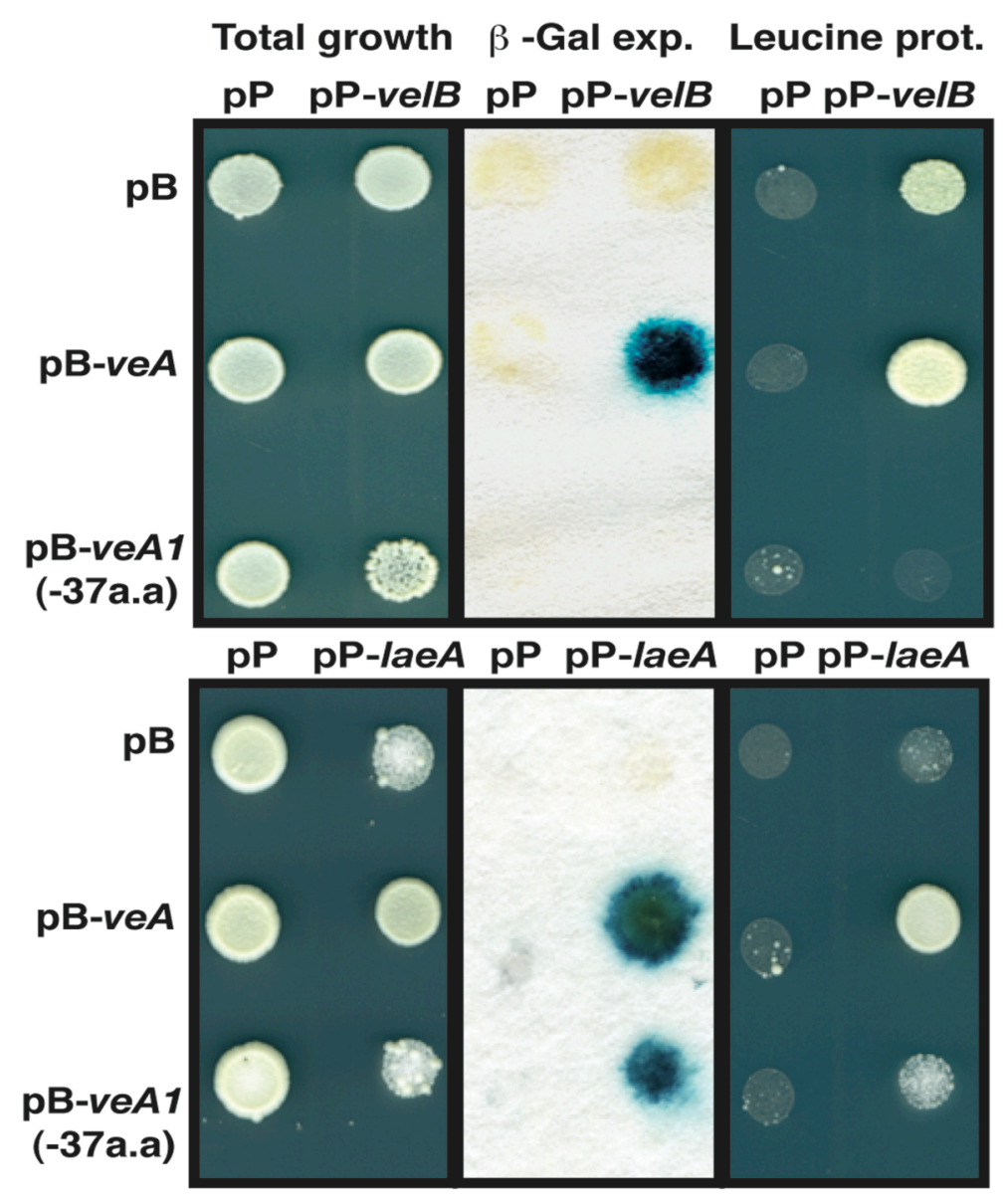

Figure 19: Interaction of VeA with VelB and LaeA in the Y2H system. Left: Total growth of yeast strains carrying plasmid combinations of $v e A, v e A 1$, velB and laeA on SC-3 (-his, -trp, -ura), 2\% galactose/1\% raffinose plates. Middle: $\beta$-galactosidase expression for strains grown on the SC-3, $2 \%$ galactose and $1 \%$ raffinose. Right: Leu prototrophy, in the pveA / pvelB strain leucine biosynthesis is restored. Despite the weak background growth on the leu-lacking media, pEG202/pJG4-5-velB is negative in $\beta$-galactosidase assays (see middle panel). pB: bait plasmid, pP: prey plasmid. 
Northern blot analysis reveals that velB expression increases in the late asexual $(24 \mathrm{~h})$ and sexual developmental stages (48, 72 h) (Fig. 20). The veA mRNA accumulation reflects this pattern (Kim et al., 2002), and suggests that VelB functions at the late asexual and sexual developmental stages, likely via the interaction with VeA.

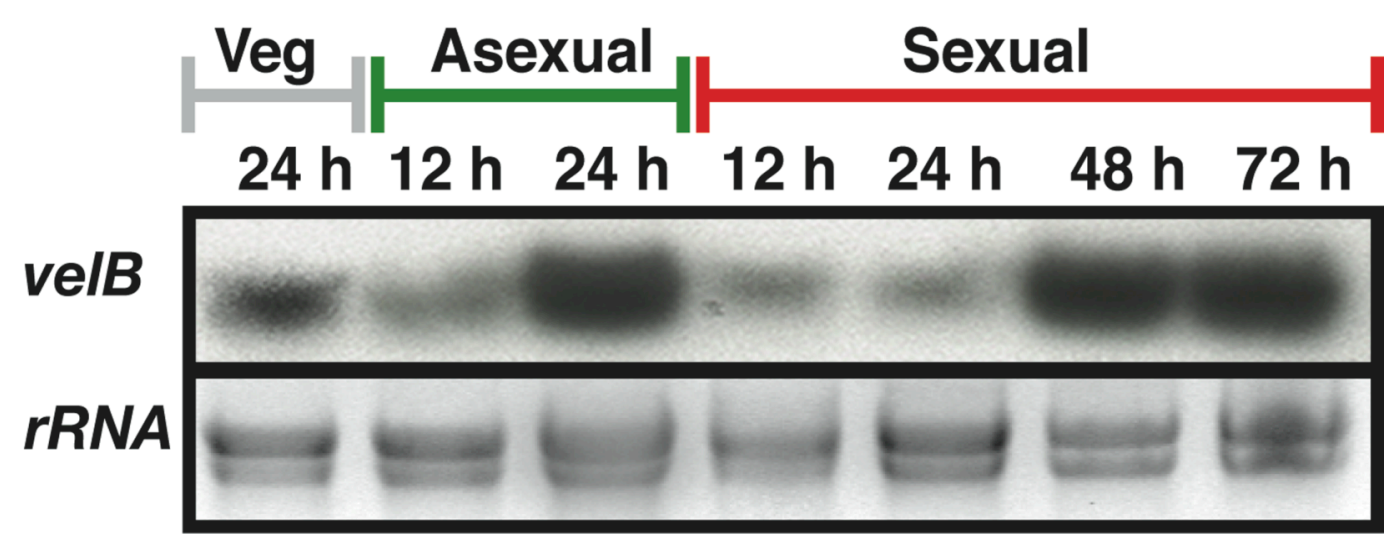

Figure 20: velB is expressed during late asexual and sexual development. Northern hybridisation shows basal expression of velB during vegetative growth $(24 \mathrm{~h})$ in submerged culture or during early periods of asexual or sexual development. Increased expression is observed as the developmental programme proceeds and reaches to the final points (in the late asexual ( $24 \mathrm{~h}$ ) and late sexual (48 and 72 hours) stages).

\section{LaeA, a global regulator of secondary metabolism, is another VeA-interacting protein}

As a third VeA interacting protein (Fig. 18A and B) we identified AN0807.3, the nuclear methyltransferase LaeA, which is required for the expression of multiple secondary metabolite biosynthetic gene clusters (Bok and Keller, 2004). Several enrichment experiments revealed that LaeA is enriched in similar amounts as VeA and VelB, suggesting a stoichiometric trimeric complex. Under illumination conditions, i.e., when VeA is weakly expressed, only VelB, but not LaeA was identified as a VeA::cTAP* interacting protein (not shown).

The VeA-LaeA interaction was further corroborated in this study by Bimolecular fluorescence complementation (BiFC) (Hu et al., 2002) of $n$-eyfp $: v e A$ and $c$-eyfp $:$ laeA in $A$. nidulans (Fig. 21). Distinct fluorescent specks indicate interactions and are visible in the nuclei, suggesting that the VeA-LaeA interaction occurs in the nucleus. The VeA1 allele lacking the N-terminal 37 amino acid failed to interact with LaeA in BiFC (not shown), which might be due to the impaired nuclear import of VeA1 (Stinnett et al., 2007). In addition, the Y2H system confirmed the interaction between VeA and LaeA. In contrast to our BiFC data, LaeA also interacted in the $\mathrm{Y} 2 \mathrm{H}$ system with the VeA1 protein, suggesting that the VeA N- 
terminus is not required for the VeA-LaeA interaction. However, LaeA did not interact with VelB in the $\mathrm{Y} 2 \mathrm{H}$ setup (not shown).

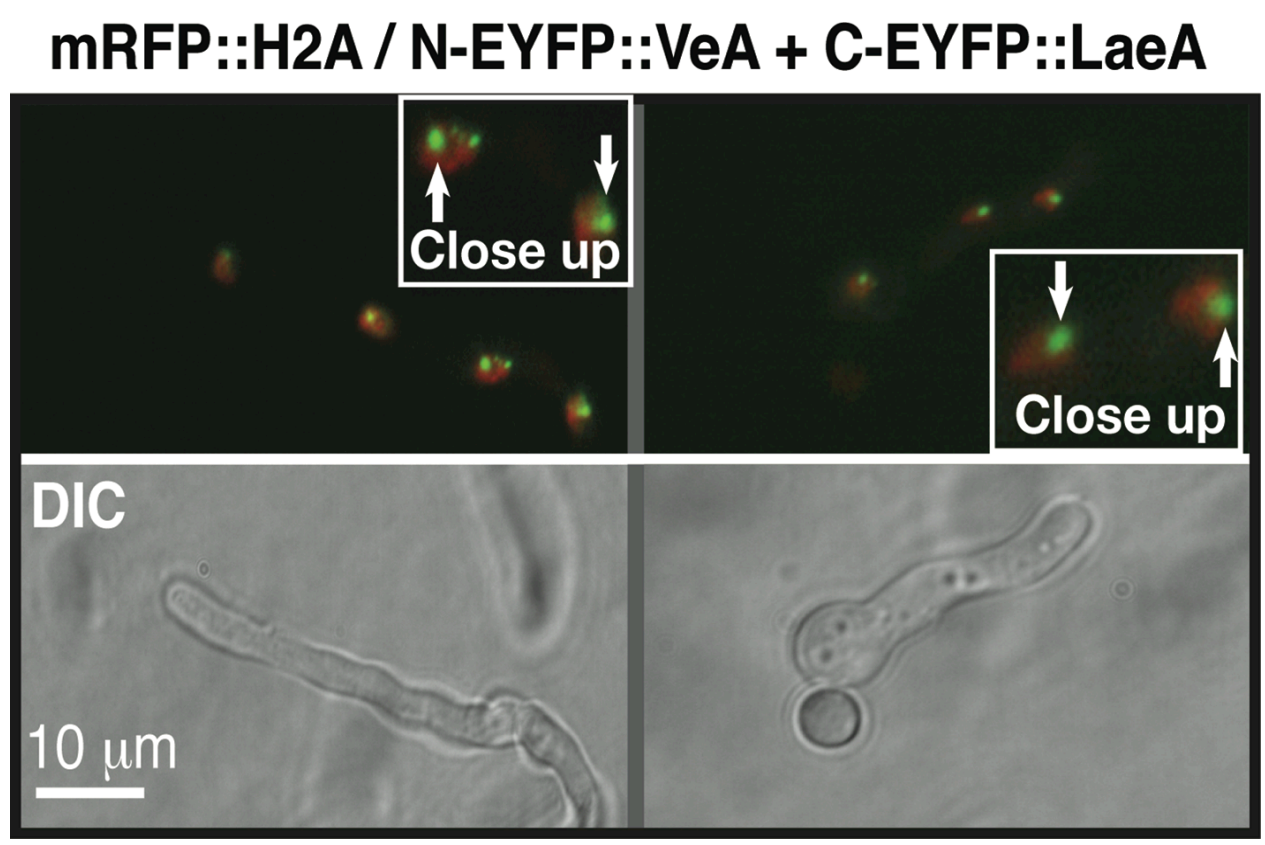

Figure 21: BiFC studies on the interaction of VeA with LaeA. N-EYFP::VeA fusion protein interacts with CEYFP::LaeA fusion protein in vivo indicated as yellow specks in the nucleus, which was counterstained with an mRFP::H2A fusion. Interaction does not take place in the whole nucleus but at certain points (gene clusters) which LaeA probably acts on.

Taken together, the VeA-LaeA complex is primarily formed in the nucleus, whereas VelB and LaeA do not show direct physical interaction. The N-terminus of VeA, which is crucial for the VeA-VelB and VeA-KapA interactions, appears to be dispensable for the VeA-LaeA interaction.

\section{The velB gene encodes a VeA-related protein}

The velB gene (Fig. 22A) has the capacity to encode a protein of 369 amino acids with a coding region made up by four exons interrupted by three intronic regions. A fourth intron is located in the 5' untranslated region of the gene. According to the Clustal W algorithm in the global alignment, the identity index between VelB and VeA is about 18\%, accompanied by many conserved amino acid substitutions in both proteins (Fig. 22B). VeA comprises a putative nuclear localization signal (NLS) sequence in its N-terminus (Kim et al., 2002) and a nuclear export signal (NES) in the middle of the protein (http://www.cbs.dtu.dk/services/NetNES/), while VelB does not have any classical NLS motif. Furthermore, VeA has a PEST (Pro, Glu or Asp, Ser, Thr rich) box responsible for 
protein degradation, but VelB appears to have only some PEST-like motifs of low value when submitted to the PEST finder internet tool (https://emb1.bcc.univie.ac.at/toolbox/pestfind/ pestfind-analysis-webtool.htm).

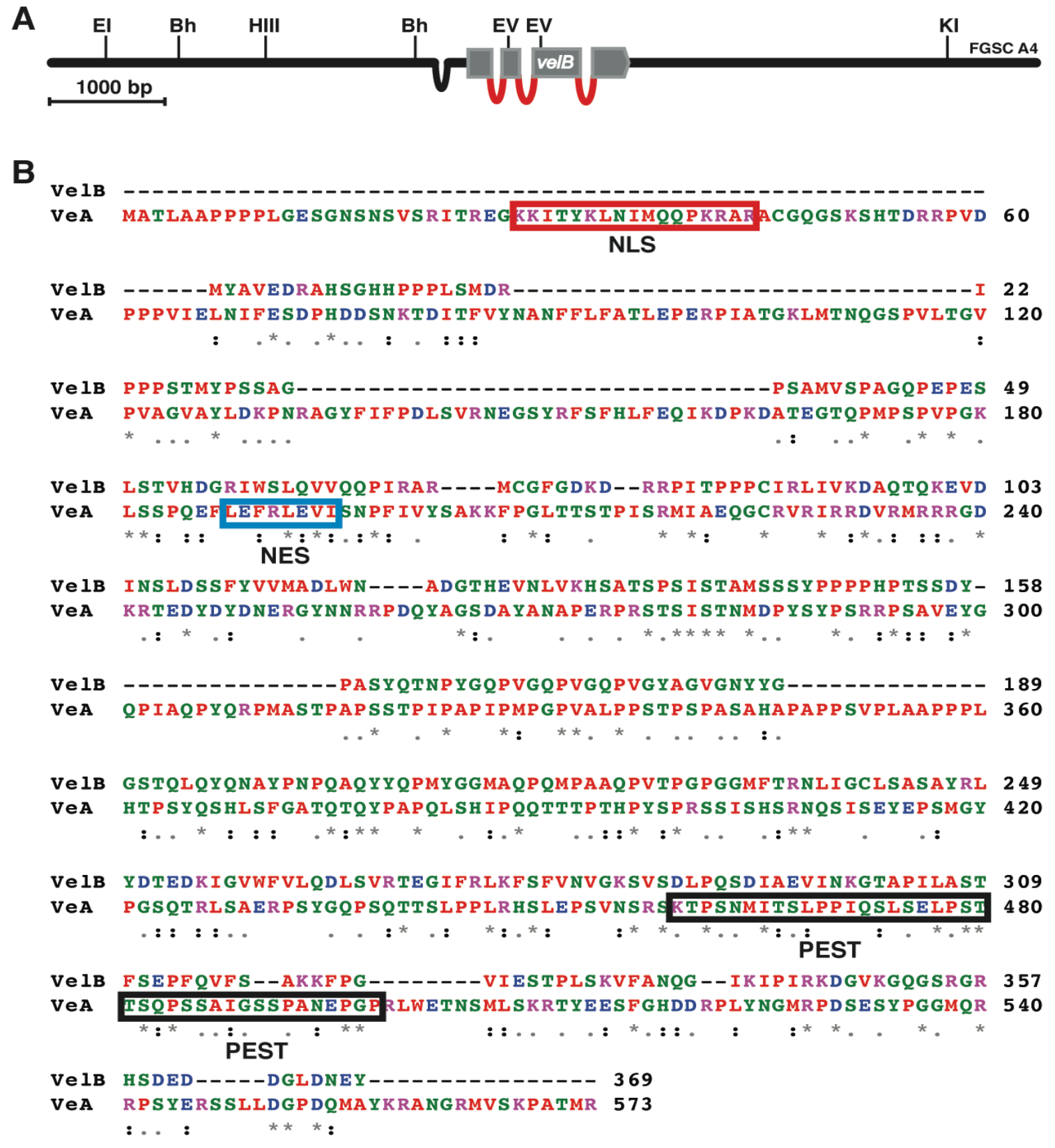

Figure 22: The velB gene structure and an alignment of VeA and VelB. (A) Architecture of the velB locus of A. nidulans. Exons are grey rectangles and recognition sites of common restriction endonucleases are given as EI (EcoRI), Bh (BamHI), HIII (HindIII), EV (EcoRV) and KI (KpnI). (B) Local alignment of the VelB and VeA proteins. Identical residues are indicated by an asterisk (*), conserved amino acid substitutions (similar amino acids) as two dots (:), and semi-conserved ones as a dot (.). The red rectangle indicates the putative nuclear localization signal (NLS) of VeA, The blue rectangle indicates the putative nuclear export signal (NES) and the black rectangle presents a conserved PEST (Pro, Glu (or Asp), Ser, Thr) motif. Red: small and hydrophobic (including aromatic amino acid), Blue: acidic, Magenta: basic, Green: hydroxyl, amine and basic amino acids. 


\section{velB $\Delta$ mutants are impaired in sexual development, sterigmatocystin production and}

\section{light response}

The evidence for the physical interaction of VeA with VelB as well as LaeA and for the formation of a heterotrimeric complex leads to the prediction that $\mathrm{VeA}$ and $\mathrm{VelB}$ are functionally interdependent. We deleted the velB gene (Fig. 23A and B) resulting in several clear defects (Fig. 24A and B). The velBA mutant no longer displays a light-dependent developmental pattern. Similar to the $v e A \Delta$ mutant, the velB $\Delta$ mutant is unable to form sexual fruit bodies even in the dark. Asexual sporulation capacity in the velB $\Delta$ mutant is impaired but not as significantly as in the veA deletion strain. Re-introduction of the velB locus fully rescued all the defects (Fig. 24A, +velB). Neither velB overexpression in a veA $\Delta$ nor veA overexpression in a velB $\Delta$ background rescued the defects of individual mutants (not shown), further corroborating the idea that VeA and VelB have an inter-dependent relationship. Interestingly, unlike overproduction of $\mathrm{VeA}$, overexpression of $v e l B$ in a $v e A^{+}$background does not cause excessive production of cleistothecia, but induces a two-fold increase in asexual sporulation in comparison to wt (not shown). The veA $\Delta / v e l B \Delta$ double mutant exhibited the phenotype identical to that of the veA $\Delta$ single mutant (not shown). These results suggest that VeA might control the number of sexual structures whereas VelB might have an additional role in asexual development.
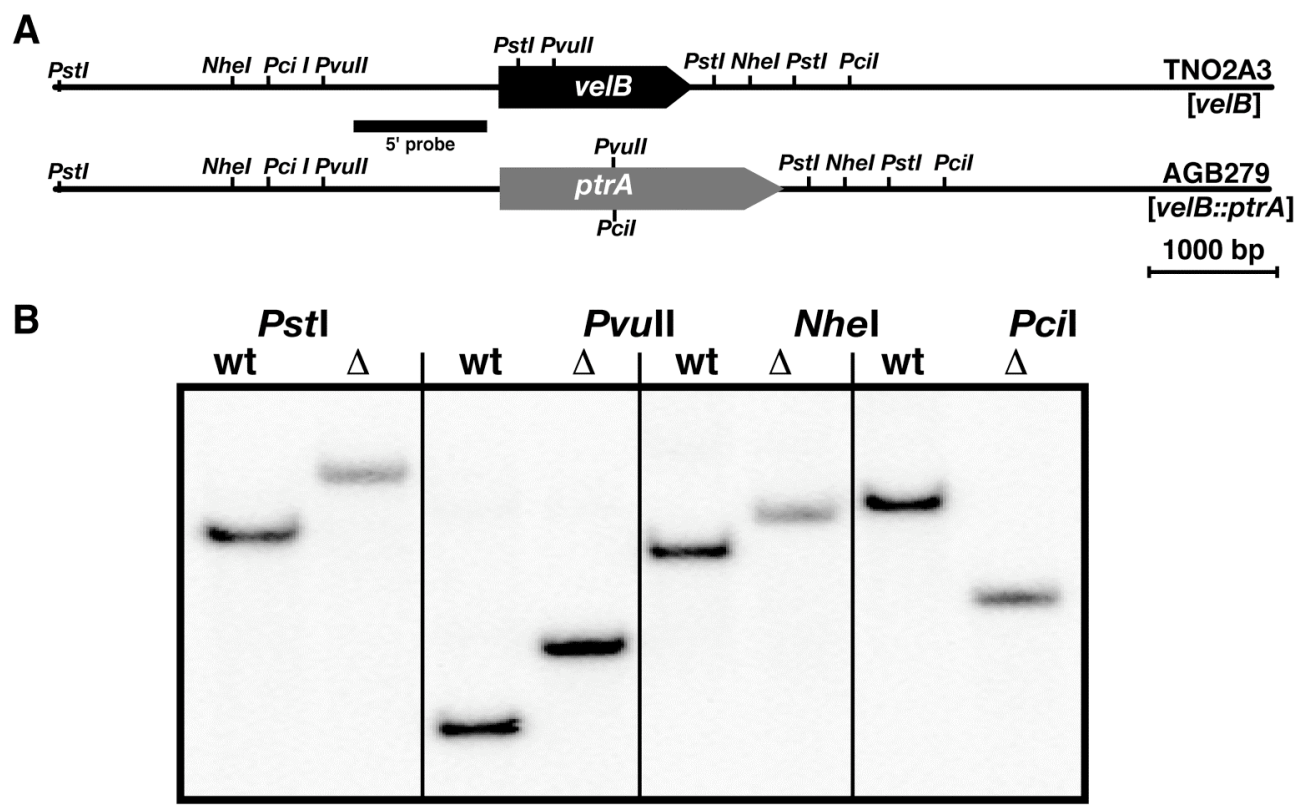

Figure 23: Deletion of velB locus via homologous gene replacement. (A) Comparative depiction of the wildtype velB locus (TNO2A3) and the velB::ptrA locus (AGB279). The black bar indicates the probe used the for Southern hybridization, which anneals to the 5 UTR region of the velB and deleted velB gene. (B) Autoradiography of Southern hybridization confirms the homologous gene replacement for the velB. 
When velB $\Delta$ strains are grown in the dark, a brownish pigment is produced, indicating the potential changes in secondary metabolism. LaeA was originally identified as a regulator of ST biosynthesis (Bok and Keller, 2004) and the veA gene is also needed for this (Kato et al., 2003). While all tested wild-type strains produce ST, no velB deletion mutant strains accumulate ST regardless of the veA allele (Fig. 24B). Therefore, VelB is an essential and novel factor, which is as much required for fungal development and secondary metabolite production as VeA. The data suggest that VelB is necessary for light-dependent secondary metabolism during sexual development presumably through controlling LaeA. This function of VelB in ST production is likely mediated by VeA, which bridges VelB and LaeA to form a heterotrimeric complex.

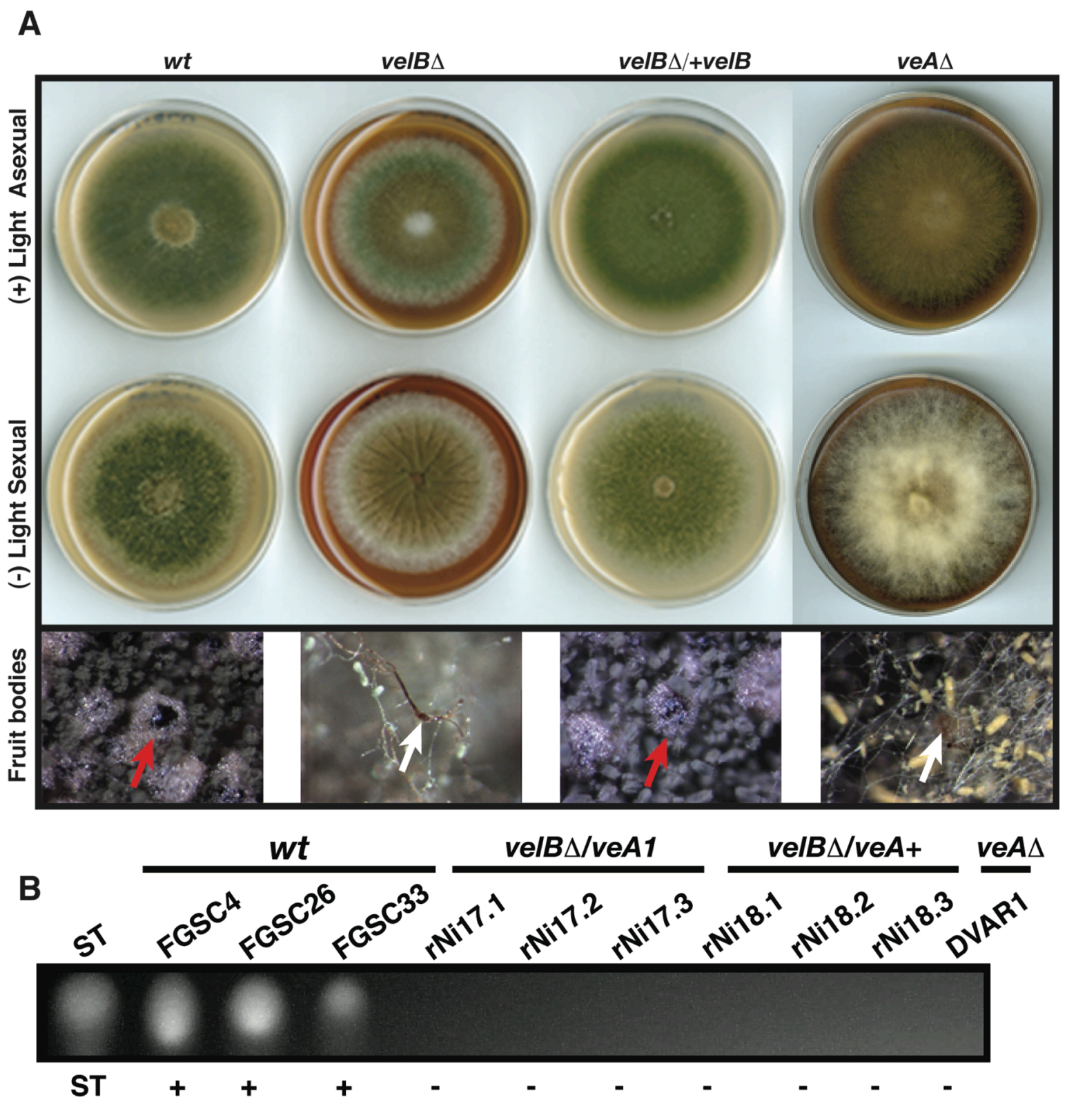


Figure 24: Deletion of $v e l B$ impairs sexual fruit body formation and sterigmatocystin production. (A) Phenotypic characterisation of velBA deletion strain AGB279. Defects are restored in the strain AGB280 (+velB). Fruit body formation in TNO2A3 and AGB280 appears as normal (red arrows), whereas aerial hyphae (white arrows) and red pigment accumulation accompanied by a lack of fruit bodies are evident for velB $\Delta$ and $v e A \Delta$ strains. Pictures of cleistothecia and hyphae were taken at 108 -fold magnification. (B) ST analysis of velB $\Delta$ strains by TLC in comparison to wt strains; in velB $\Delta$ strains ST cannot be detected regardless of the veA alleles, as a negative control no detection in DVAR1 (veA4) strain while in wt strains FGSC4 (wt, veA+), FGSC26 and FGSC33 (veAl) ST is detected. ST: ST standard.

\section{In contrast to LaeA which is constitutively nuclear and VeA which is light-dependently localized, VelB is in the nucleus as well as in the cytoplasm}

The BiFC data (Fig. 21) suggest that the interaction of VeA and LaeA occurs in the nucleus exclusively. Thus, in order to form a trimeric complex, VelB must be able to enter the nucleus despite the lack of an obvious NLS. The cellular localization of the LaeA fusion protein in the dark was nuclear as described (Bok and Keller, 2004) and did not significantly change in the absence or presence of either VeA or VelB (Fig. 25A). No changes in the LaeA nuclear localization during illumination were observed (not shown).

The distribution of the functional VelB fusion protein appears to be more complicated, because it was found in both the nucleus and the cytoplasm (Fig. 25B). This prompted us to test whether VelB localization is dependent on illumination as it has been described for VeA (Stinnett et al., 2007). The cellular localization of VelB did not significantly change when the fungus was grown in liquid culture under daylight or in the dark. Moreover, the exposure of the fungal cells to air, which normally induces differentiation, did not affect the ubiquitous localization of VelB in the cell (Fig. 25B).

A functional veA::sgfp fusion supports recent findings (Stinnett et al., 2007) that VeA localization is affected by illumination (Fig. 25C): VeA is predominantly cytoplasmic and only weakly nuclear when incubation was carried out in liquid culture under day light conditions but enriched in the nucleus when the fungus is incubated in liquid medium in the dark. Cultivation in the absence of light and under restricted air-exposure results in most efficient nuclear accumulation of the fusion protein probably correlated with the onset of the sexual differentiation programme and with increased secondary metabolism.

Taken together, these data suggest that LaeA, VelB and VeA all have nuclear subpopulations of different sizes. LaeA seems to be primarily present in the nucleus and its cellular localization does not depend on either VeA or VelB. Whereas VeA is enriched in the nucleus in the dark, VelB shows both a substantial cytoplasmic as well as a nuclear subpopulation. 

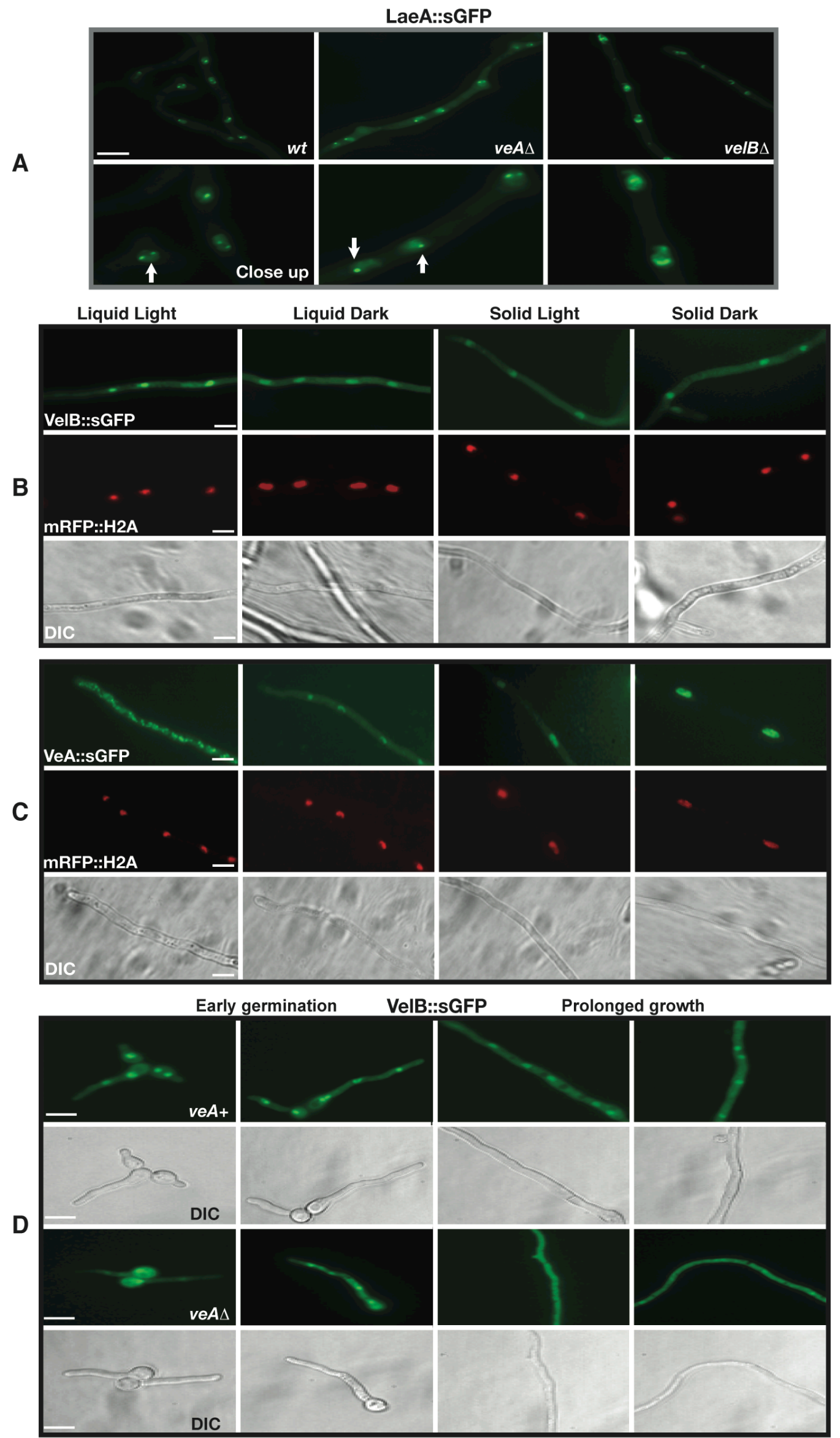
Figure 25: VeA enhances the nuclear transport of VelB. (A) Localization of LaeA:ssGP in wt, veA $\Delta$ and velBA backgrounds. (B) A functional VelB::sGFP (AGB277, AGB281). (C) Localization of a functional VeA::sGFP. (D) Fluorescence patterns in strains AGB277 and AGB275 expressing velB::sgfp in veA+ and veAA backgrounds. Bars represent $10 \mu \mathrm{m}$.

\section{VeA enhances the nuclear localization of VelB}

The next question we addressed was whether the nuclear localization of VelB lacking a canonical NLS is affected by VeA or whether there is an atypical NLS signal within VelB. Accordingly, we tested whether localization of VelB depends on VeA or vice versa. When VeA is present, germination of the conidiospores at about 10-12 hours after inoculation (early stage) and hyphae formation after 18 hours (late stage) resulted in the accumulation of the functional VelB::sGFP fusion protein in the nucleus (Fig. 25D). In contrast, localisation of the VelB::sGFP fusion protein in a $v e A \Delta$ background is almost exclusively cytoplasmic with some weak nuclear localization that becomes even weaker in the elongated hyphae. Collectively, we propose that VelB predominantly localizes to the nucleus with the assistance of VeA. When VeA is absent, a residual VelB nuclear transport is still present which might depend on an unconventional NLS or some other proteins. A comparison of VeA localization in the presence or absence of VelB suggests that the presence of VelB delays the nuclear transport of VeA, further supporting that nuclear import of VeA and VelB can affect each other (not shown).

Our data suggest that the mechanism underlying the coordinated induction of sexual development and secondary metabolism in A. nidulans might be the interaction between the key developmental regulatory complex VelB/VeA and LaeA. We propose that in the dark the VelA/VeA/LaeA interaction controls and presumably supports the epigenetic activity of LaeA, which subsequently results in increased expression of secondary metabolite gene clusters. In light, this interaction is inhibited because the bridging factor VeA is unable to enter the nucleus. Since the absence of LaeA does not result in clear developmental changes, the VelB/VeA complex may have additional functions in the fungal development. 


\section{Discussion}

Fungal development and metabolism respond to various external signals including a wide spectrum of wavelengths of light. A. nidulans produces many compounds relevant to biotechnology and human health and is a well-suited model for the analysis of the interplay between secondary metabolism, light and differentiation. The novel fungal protein, VelB in conjunction with $\mathrm{VeA}$, connects light-responding development and secondary metabolism in A. nidulans. VelB is linked by direct protein interactions to the light-inhibited VeA and to the global regulator of secondary metabolism LaeA (Fig. 26). We present evidence that the formation of this complex is the molecular basis that synchronises developmental and metabolic changes to the disappearance of light. Various VelB functions depend on the lightcontrolled cellular context, which allows or prohibits the interaction with the other two proteins.

A. nidulans grows vegetatively in the soil by hyphal tip extension through which it gains the competence for development and secondary metabolism (Adams et al., 1998). The light trigger of asexual development corresponds to its needs as a terrestrial inhabitant to release high numbers of asexual spores (conidia) to the air. Vegetative growth and asexual development do not require VeA or LaeA extensively, but require VelB for maximal yields of conidia. These conditions correlate with the light-dependent cytoplasmic localization of VeA, the constitutive nuclear function of LaeA, and the partial nuclear localization of VelB, respectively. The basal level VelB import into the nucleus does not require VeA. In light, when only little amounts of $\mathrm{VeA}$ and VelB are present in the nucleus, the nuclear master regulator of secondary metabolism LaeA seems to be only active in those hyphae that are not exposed to light. Accordingly, the deletion of laeA results in a loss of mycelial pigmentation at the bottom of the colony (Bok and Keller, 2004).

VeA possesses a classical NLS (Stinnett et al., 2007), and therefore the low amounts of cytoplasmic protein found under illumination require light-dependent inhibition of nuclear import. VeA localization is reminiscent to the phytochrome-dependent localization of plant COP1, a repressor of plant photomorphogenesis, which is also localized in the cytoplasm in the light (von Arnim and Deng, 1994). Fungal VeA co-localizes in the cytoplasm with the constitutively cytoplamic red light receptor FphA (Blumenstein et al., 2005; Purschwitz et al., 2006). It is intriguing to speculate that light changes the FphA activity and this results in inactivation of the VeA NLS site and therefore its exclusion from the nucleus.

During sexual development and in the dark, VeA and VelB are highly produced. VeA is transported into the nucleus and enhances the nuclear import of VelB. This seems to be the 
key molecular event that induces sexual development and the elevated production of the LaeA-dependent secondary metabolites. Sexual development requires a series of fundamental changes in metabolism, which result in formation of different cell and tissue types in $A$. nidulans (Braus et al., 2002b). Sexual development and the formation of many secondary metabolites occur after asexual development, and prefer limited air exchange, solid surface and the darkness (Axelrod et al., 1973).

LaeA is required for secondary metabolism but not for sexual development, whereas VeA and VelB are required for both. This hints to LaeA-independent functions of VelB/VeA in sexual development. Overexpression studies suggest that the amount of VeA determines the developmental decisions in A. nidulans, whereas enhanced expression of VelB does not affect sexual development. Localization studies imply that VelB does not influence the intracellular shuttling of VeA between cytoplasm and nucleus, and that VelB is constitutively present in both cellular compartments. Interaction between VeA and VelB depends on the VeA N-terminus which is also required for the $\alpha$-importin KapA interaction and nuclear import (Stinnett et al., 2007). Nuclear localization of VelB depends to a great extent on VeA, indicating that the cytoplasmic VelB/VeA dimer translocates into the nuclear compartment via the VeA NLS. We assume, that a certain threshold level of the VelB/VeA complex, which is reached only in the dark, has to be present in the nucleus to promote further development.

Since neither VeA nor VelB carries any obvious DNA binding or catalytic motif, VelB/VeA might have additional, presumably more transient, interacting partners besides LaeA. For instance, the VelB/VeA complex might be involved in the degradation of lightinduced regulators. The Arabidopsis thaliana COP1 photomorphogenesis repressor, which shows a VeA-like light dependent cellular distribution (Ulm and Nagy, 2005), has an E3 ubiquitin ligase activity. COP1 targets phytochrome and light-dependent transcription factors to the protein destruction machinery (Seo et al., 2004a). The COP1 action requires the COP9 signalosome (CSN) protein complex, which is also present in fungi. We have shown that defects in the fungal COP9 signalosome result in light independent constitutive progression of sexual development (Busch et al., 2003). The precisely controlled protein degradation is known to be important for the formation of ascospores in A. nidulans (Krappmann et al., 2006). This opens a scenario where the VelB/VeA heterodimer bridges proteins which support the destruction of factors required during illumination. VeA does not seem to physically interact with CSN (not shown) and therefore the connection between light control and CSN remains to be elucidated. 
Despite the inability of VelB to interact with LaeA directly, the absence of velB impairs both sexual development and secondary metabolite production. This leads to the idea that VelB affects LaeA indirectly by interacting with VeA, which in turn bridges VelB and LaeA. A consequence of the formation of a trimeric complex may include sequestering VelB from its role in asexual development. The LaeA-VeA interaction seems to be independent of the N-terminus of VeA where VelB binds, which further supports the physically ordered trimeric VelB/VeA/LaeA complex. We propose to designate this trimeric complex, which coordinates sexual development and secondary metabolism in response to light, the velvet complex. What is the function of the interaction between the VelB/VeA complex and LaeA? We assume that VelB/VeA is part of the epigenetic control of chromatin remodeling by modulating the LaeA methyltransferase activity which has been suggested to modify histones (Bok and Keller, 2004; Keller et al., 2005). When we cross-linked the VeA-TAP* to fungal extracts by formaldehyde we identified the histone H2B in addition to LaeA and VelB (not shown). This result further corroborates that $\mathrm{VelB} / \mathrm{VeA} / \mathrm{LaeA}$-mediated control occurs at the level of histone modification.

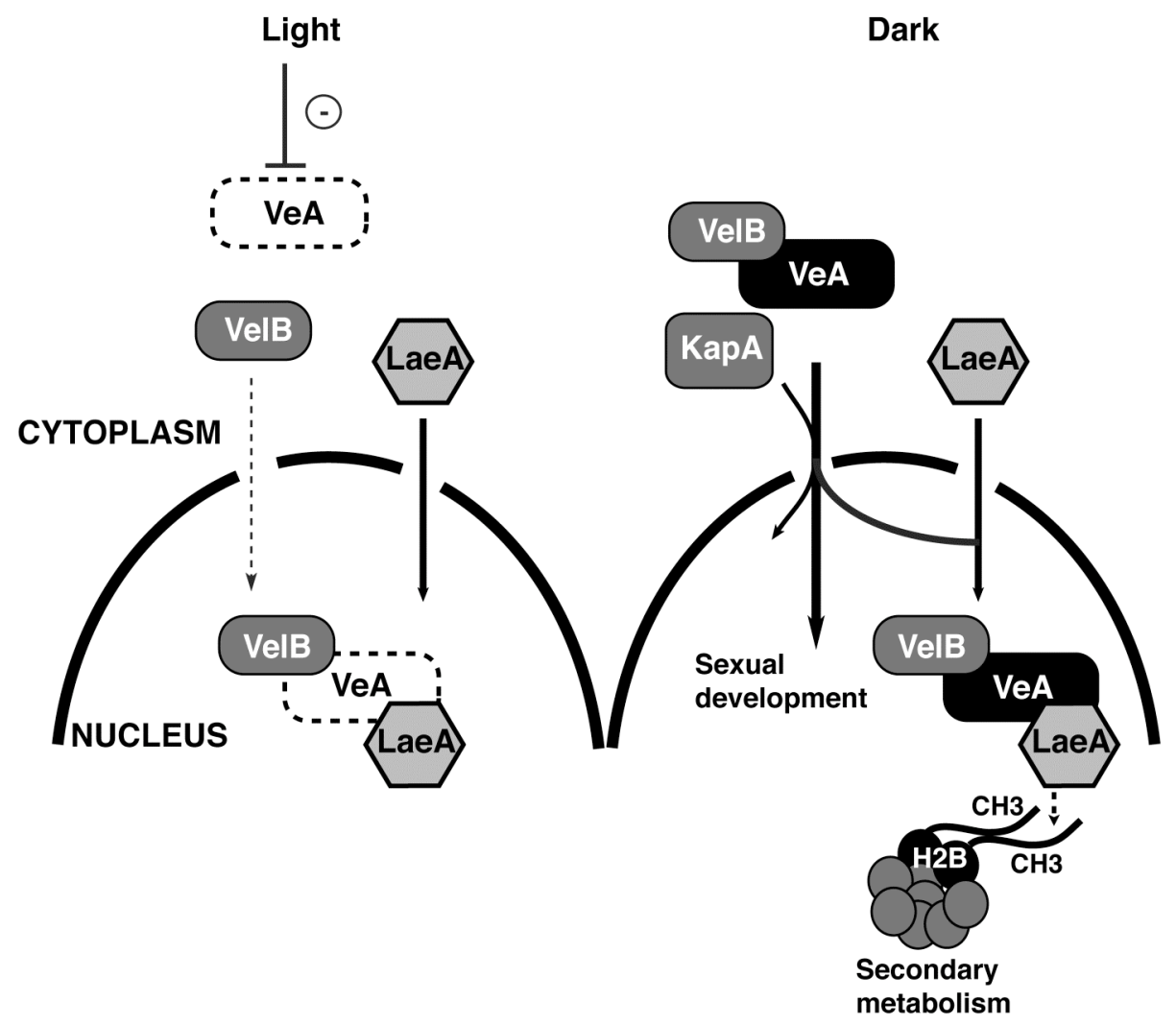

Figure 26: Model for the velvet complex action. In light: low amounts of VeA are retained in the cytoplasm; VelB supports asexual spore formation and LaeA shows low activity. In the dark: abundant VeA is transported into the nucleus together with KapA and VelB. VelB/VeA and LaeA form the velvet complex that epigenetically activates secondary metabolite clusters; at least VelB/VeA are required for sexual development. 
Conclusively, we propose (Fig. 26) that more amounts of VeA is functionally active in the dark and forms a complex with increased amounts of VelB. When the VeA enhanced nuclear transport of VelB has reached a threshold, the velvet compex is formed to control LaeA and to initiate sexual development. Fungal morphogenesis and secondary metabolism have traditionally been kept as separate fields, yet these are obviously inter-connected. Our studies of the $\mathrm{VelB} / \mathrm{VeA} / \mathrm{LaeA}$ complex elucidate the molecular mechanisms underlying the intimate relationship between fungal development and secondary metabolism. 


\section{References}

Adams, T.H., Wieser, J.K., and Yu, J.H. (1998). Asexual sporulation in Aspergillus nidulans. Microbiol Mol Biol Rev 62, 35-54.

Axelrod, D.E., Gealt, M., and Pastushok, M. (1973). Gene control of developmental competence in Aspergillus nidulans. Dev Biol 34, 9-15.

Blumenstein, A., Vienken, K., Tasler, R., Purschwitz, J., Veith, D., Frankenberg-Dinkel, N., and Fischer, R. (2005). The Aspergillus nidulans phytochrome FphA represses sexual development in red light. Curr Biol 15, 1833-1838.

Bok, J.W., and Keller, N.P. (2004). LaeA, a regulator of secondary metabolism in Aspergillus spp. Eukaryot Cell 3, 527-535.

Bok, J.W., Noordermeer, D., Kale, S.P., and Keller, N.P. (2006). Secondary metabolic gene cluster silencing in Aspergillus nidulans. Mol Microbiol 61, 1636-1645.

Brakhage, A.A. (1997). Molecular regulation of penicillin biosynthesis in Aspergillus (Emericella) nidulans. FEMS Microbiol Lett 148, 1-10.

Braus, G.H., Krappmann, S., and Eckert, S. (2002). Sexual development in ascomycetes: Fruit body formation in Aspergillus nidulans. In Molecular Biology of Fungal Development, Osiewacz, ed (New York: Marcel Dekker Inc), pp. 215-244.

Brown, T., and Mackey, K. (1997). Analysis of RNA by Northern and Slot Blot Hybridization. In Current Protocols in Molecular Biology (John Wiley \& Sons Inc.), pp. 4.9.1-4.9.16.

Busch, S., Eckert, S.E., Krappmann, S., and Braus, G.H. (2003). The COP9 signalosome is an essential regulator of development in the filamentous fungus Aspergillus nidulans. Mol Microbiol 49, 717-730. 
Calvo, A.M., Wilson, R.A., Bok, J.W., and Keller, N.P. (2002). Relationship between secondary metabolism and fungal development. Microbiol Mol Biol Rev 66, 447-459, table of contents.

Chaveroche, M.K., Ghigo, J.M., and d'Enfert, C. (2000). A rapid method for efficient gene replacement in the filamentous fungus Aspergillus nidulans. Nucleic Acids Res 28, E97.

Cole, R.a.S., M. (2003). Handbook of Secondary Fungal Metabolites. (Amsterdam: Elsevier).

Feinberg, A.P., and Vogelstein, B. (1983). A technique for radiolabeling DNA restriction endonuclease fragments to high specific activity. Anal Biochem 132, 6-13.

Golemis, and Brent, R. (1996). In Current protocols in molecular biology, F.M. Ausubel, R. Brent, R.E. Kingston, D.D. Moore, J.G. Seidmann, A.J. Smith, and K. Struhl, eds (Massachusetts: Harvard Medical School), pp. 429-454.

Gyula, P., Schafer, E., and Nagy, F. (2003). Light perception and signalling in higher plants. Curr Opin Plant Biol 6, 446-452.

Gyuris, J., Golemis, E., Chertkov, H., and Brent, R. (1993). Cdi1, a human G1 and S phase protein phosphatase that associates with Cdk2. Cell 75, 791-803.

Hanahan, D., Jessee, J., and Bloom, F.R. (1991). Plasmid transformation of Escherichia coli and other bacteria. Methods Enzymol 204, 63-113.

Hicks, J., Shimizu, K. \& Keller, N. (2002). Genetics and biosynthesis of aflatoxins and sterigmatocystin. In The Mycota, F. Kempken, ed (Berlin: Springer-Velag), pp. 55-69.

Hu, C.D., Chinenov, Y., and Kerppola, T.K. (2002). Visualization of interactions among bZIP and Rel family proteins in living cells using bimolecular fluorescence complementation. Mol Cell 9, 789-798. 
Kato, N., Brooks, W., and Calvo, A.M. (2003). The expression of sterigmatocystin and penicillin genes in Aspergillus nidulans is controlled by veA, a gene required for sexual development. Eukaryot Cell 2, 1178-1186.

Keller, N.P., Turner, G., and Bennett, J.W. (2005). Fungal secondary metabolism - from biochemistry to genomics. Nat Rev Microbiol 3, 937-947.

Kim, H., Han, K., Kim, K., Han, D., Jahng, K., and Chae, K. (2002). The veA gene activates sexual development in Aspergillus nidulans. Fungal Genet Biol 37, 72-80.

Kircher, S., Gil, P., Kozma-Bognar, L., Fejes, E., Speth, V., Husselstein-Muller, T., Bauer, D., Adam, E., Schafer, E., and Nagy, F. (2002). Nucleocytoplasmic partitioning of the plant photoreceptors phytochrome $\mathrm{A}, \mathrm{B}, \mathrm{C}, \mathrm{D}$, and $\mathrm{E}$ is regulated differentially by light and exhibits a diurnal rhythm. Plant Cell 14, 1541-1555.

Kolar, M., Punt, P.J., van den Hondel, C.A., and Schwab, H. (1988). Transformation of Penicillium chrysogenum using dominant selection markers and expression of an Escherichia coli lacZ fusion gene. Gene 62, 127-134.

Krappmann, S., Bayram, O., and Braus, G.H. (2005). Deletion and allelic exchange of the Aspergillus fumigatus veA locus via a novel recyclable marker module. Eukaryot Cell 4, 1298-1307.

Krappmann, S., Jung, N., Medic, B., Busch, S., Prade, R.A., and Braus, G.H. (2006). The Aspergillus nidulans F-box protein GrrA links SCF activity to meiosis. Mol Microbiol 61, 76-88.

Kunkel, T.A. (1985). Rapid and efficient site-specific mutagenesis without phenotypic selection. Proc Natl Acad Sci U S A 82, 488-492.

Li, S., Myung, K., Guse, D., Donkin, B., Proctor, R.H., Grayburn, W.S., and Calvo, A.M. (2006). FvVE1 regulates filamentous growth, the ratio of microconidia to macroconidia and cell wall formation in Fusarium verticillioides. Mol Microbiol 62, 1418-1432. 
Lin, C., and Todo, T. (2005). The cryptochromes. Genome Biol 6, 220.

Muyrers, J.P., Zhang, Y., and Stewart, A.F. (2000). ET-cloning: think recombination first. Genet Eng (N Y) 22, 77-98.

Nayak, T., Szewczyk, E., Oakley, C.E., Osmani, A., Ukil, L., Murray, S.L., Hynes, M.J., Osmani, S.A., and Oakley, B.R. (2006). A versatile and efficient gene-targeting system for Aspergillus nidulans. Genetics 172, 1557-1566.

Punt, P.J., and van den Hondel, C.A. (1992). Transformation of filamentous fungi based on hygromycin B and phleomycin resistance markers. Methods Enzymol 216, 447-457.

Purschwitz, J., Muller, S., Kastner, C., and Fischer, R. (2006). Seeing the rainbow: light sensing in fungi. Curr Opin Microbiol 9, 566-571.

Rohila, J.S., Chen, M., Cerny, R., and Fromm, M.E. (2004). Improved tandem affinity purification tag and methods for isolation of protein heterocomplexes from plants. Plant J 38, 172-181.

Saiki, R.K., Bugawan, T.L., Horn, G.T., Mullis, K.B., and Erlich, H.A. (1986). Analysis of enzymatically amplified beta-globin and HLA-DQ alpha DNA with allele-specific oligonucleotide probes. Nature 324, 163-166.

Sambrook, J., Fritsch, E.F., and Maniatis, T. (1989). Molecular Cloning: A Laboratory Manual. (Cold Spring Harbor, NY: Cold Spring Harbor Laboratory Press).

Seiler, S., Vogt, N., Ziv, C., Gorovits, R., and Yarden, O. (2006). The STE20/Germinal Center Kinase POD6 Interacts with the NDR Kinase COT1 and Is Involved in Polar Tip Extension in Neurospora crassa. Mol Biol Cell 17, 4080-4092.

Seo, H.S., Watanabe, E., Tokutomi, S., Nagatani, A., and Chua, N.H. (2004). Photoreceptor ubiquitination by COP1 E3 ligase desensitizes phytochrome A signaling. Genes Dev 18, 617-622. 
Shevchenko, A., Wilm, M., Vorm, O., and Mann, M. (1996). Mass spectrometric sequencing of proteins silver-stained polyacrylamide gels. Anal Chem 68, 850-858.

Southern, E.M. (1975). Detection of specific sequences among DNA fragments separated by gel electrophoresis. J Mol Biol 98, 503-517.

Stinnett, S.M., Espeso, E.A., Cobeno, L., Araujo-Bazan, L., and Calvo, A.M. (2007). Aspergillus nidulans VeA subcellular localization is dependent on the importin alpha carrier and on light. Mol Microbiol 63, 242-255.

Thompson, J.D., Higgins, D.G., and Gibson, T.J. (1994). CLUSTAL W: improving the sensitivity of progressive multiple sequence alignment through sequence weighting, position-specific gap penalties and weight matrix choice. Nucleic Acids Res 22, 4673 4680.

Ulm, R., and Nagy, F. (2005). Signalling and gene regulation in response to ultraviolet light. Curr Opin Plant Biol 8, 477-482.

von Arnim, A.G., and Deng, X.W. (1994). Light inactivation of Arabidopsis photomorphogenic repressor COP1 involves a cell-specific regulation of its nucleocytoplasmic partitioning. Cell 79, 1035-1045.

Woloshuk, C.P., Foutz, K.R., Brewer, J.F., Bhatnagar, D., Cleveland, T.E., and Payne, G.A. (1994). Molecular characterization of $a f l R$, a regulatory locus for aflatoxin biosynthesis. Appl Environ Microbiol 60, 2408-2414.

Yager, L.N. (1992). Early developmental events during asexual and sexual sporulation in Aspergillus nidulans. Biotechnology 23, 19-41.

Yu, J.H., Hamari, Z., Han, K.H., Seo, J.A., Reyes-Dominguez, Y., and Scazzocchio, C. (2004). Double-joint PCR: a PCR-based molecular tool for gene manipulations in filamentous fungi. Fungal Genet Biol 41, 973-981. 
Zonneveld, B.J.M. (1977). Biochemistry and ultrastructure of sexual development in Aspergillus. (London: Academic Press). 


\section{Conclusions}

\section{Fungal CryA: the missing link between cryptochromes and photolyases}

Light perception is an enigmatic process that directs many genetic, physiological and biochemical processes for the optimal growth of various organisms. Fungi need day light for their proper spore production. Molecular networks comprising light perception and transduction of the light signal to gene expression are poorly understood. As a part of the blue light sensing machinery, specialized receptors receive the blue light spectrum and regulate gene expression. Two important protein families, the cryptochromes and the photolyases are closely related and demonstrate high indentity to each other. However, with respect to function they appear as highly diverged. DNA photolyases repair UV-damaged DNA by activation of UVA light (Sancar, 2000; Green, 2004; Lin and Todo, 2005). Cryptochromes (CRYs) have more specialized roles such as in the entrainment of the circadian rhythm or the daily rhythm in many organisms, flowering, cell elongation, leaf expansion in plants in response to the blue light spectrum. Importantly, they lack DNA repair activity (Sancar, 2000; Lin and Todo, 2005). CRYs are thought to have evolved from DNA photolyases by gene duplication and have lost their DNA repair activities (Sancar, 2000). So far, no true cryptochrome has been studied in fungal organisms, only phototropins termed as WHITE COLLAR-1 and -2 have been studied mostly in N. crassa. They regulate many responses such as the entrainment of the circadian clock, caretenoid synthesis, protoperithecia and photo induction (Ballario et al., 1996; Ballario and Macino, 1997; Dunlap and Loros, 2004).

In this work, the cryptochrome/photolyase encoding gene of $A$. nidulans, cryA, as first fungal cryptochrome was investiagated and its role in blue light signaling pathway was analysed. The cryA gene was deleted and expressed in a heterologous E.coli host defective in UV-induced DNA repair. CryA displays photoreversal activity in E.coli, i.e., it repairs DNA damage upon UVA light illumination. Forced expression under nitrate promoter (Muro-Pastor et al., 1999) contributes to the survival in A. nidulans. cryA, despite being classified as a photolyase-like protein, controls sexual development-specific gene expression. Experimental data reveal that cryA is a cryptochrome that still holds photolyase function or that has not been evolved completely. The crystal structure of CryA and extensive comparison of its crystal structure to the other members of cryptochrome/photolyase family can give more insight into the dual function of CryA. Investigation of CryA-like proteins in closely related fungi will shed light on the evolution of fungal cryptochromes in detail. Moreover, application of affinity purifications employing the TAP tag might help to uncover the proteins with which CryA interacts in the cell. 


\section{Velvet proteins are highly conserved in ascomycetous fungi}

Recent publications of fungal genomes revealed conserved sequences. Velvet proteins are structurally conserved throughout ascomycetes. The genome sequences of N. crassa and A. nidulans have been determined (Galagan et al., 2003; Galagan et al., 2005) and inspection of both genomes reveals many orthologous sequences, the function of which might be conserved or altered during the course of evolution.

Here, we have characterized gene of $N$. crassa orthologous to velvet $A$ performing gene disruption, expression and cross-complementation experiments. Alignment of the two proteins disclosed the fact that velvet proteins are highly similar in structure and this similarity is more pronounced in the N-terminus of the protein. Deletion of the $N$. crassa ve- 1 uncovered that $v e-1$ bears a repressor function in asexual conidiation while it does not have any sexual development function in $N$. crassa. The sporulation phenotype is not light dependent but expression of ve-1 increases under red light illumination. Crosscomplementation experiments unveiled that $N$. crassa velvet is functional in A. nidulans both in terms of promoter and coding sequence. This is the first study showing the functional conservation between two velvet proteins of two different organisms. The role of ve-1 in $N$. crassa is to regulate asexual sporulation probably due to different downstream elements or a redundancy in the sexual development pathway, whereas ve-l's role in sexual development remains elusive. It will be interesting to see if VE-1 is a part of the red light signaling network because the expression, or at least the promoter of ve-l is responsive to red light. The functions of the red light receptor encoding genes phy-1 and phy-2 in N. crassa is still unclear (Froehlich et al., 2005). It will be attractive to perform in vivo and in vitro interaction experiments between VE-1 and Neurospora phytochromes. The $N$. crassa genome contains a second velvet protein encoding sequence, which brings the question into mind if ve-2 has any redundant function that takes over the role of $v e-1$ in the absence of $v e-1$. To answer this question, it will be necessary to create and analyse the double mutants (ve-1 $1 / v e-2 \Delta)$. More detailed analysis at the protein level as has been performed for the veA gene product of A. nidulans might yield insights into the fundemental differences and similarities between velvet-mediated developmental programmes in two different organisms.

\section{The Velvet complex : A core element that regulates light-dependent development and secondary metabolism in A. nidulans}

The homothallic ascomycete fungus $A$. nidulans undergoes asexual and sexual stages, both of which require certain gene products and environmental signals (Adams et al., 1998; Braus et 
al., 2002). The velvet protein, which is responsible for the light-dependent sexual development, integrates the environmental input (light) into a developmental program and regulates the balance between asexual conidiation and sexual development (Käfer, 1965; Champe et al., 1981; Mooney and Yager, 1990; Timberlake, 1990; Kim et al., 2002). It was also claimed that it participates in secondary metabolism to some extent (Kato et al., 2003). However, the mode of velvet action remained elusive. Protein complexes formed by different proteins are the key players of cellular physiology, metabolism and signal transduction and act as transcription factors, signaling complexes, enzymes and cytoskeletal elements.

In this work, we targeted the VeA interactome by applying the commonly used biochemical TAP tag purification method with a codon-optimized tag (Puig et al., 2001; Busch et al., 2007). We were able to co-purify three proteins VelB, LaeA and KapA along with VeA::TAP* as VeA-interacting proteins from dark grown cultures. VelB was identified as velvet-like protein because of its similarity to VeA. LaeA is described as a master regulator of secondary metabolism (Bok and Keller, 2004), and KapA is an alpha importin which was already shown to interact with VeA (Stinnett et al., 2007). We have been able to demonstrate that there is a velvet complex which simultaneously regulates light-dependent sexual development and ST production by activating the putative methyl transferase LaeA. This might answer the phenomenon that leads to the loss of ST production and down-regulation of other secondary metabolite genes in veAs background. The velvet complex covers an important position in the cell signalling network of Aspergillus nidulans. Similar complexes are likely to be found in different filamentous fungi because the components of this complex are highly conserved among filamentous ascomycetes. From now on, it will be interesting to know which additional proteins are included in this complex and which proteins interact with VelB and LaeA alone. Therefore to tag these two proteins with TAP tag will provide information about possible downstream elements. Altough it is reported that VeA interacts with the FphA phytochrome in yeast two-hybrid system (Purschwitz et al., 2006). The connection between the velvet complex and the two main light receptors, phytochrome FphA and cryptochrome CryA, has not been studied substantially. Hence, the future studies will focus on the connection between light receptors and the velvet complex. For that aim, tagging these two light receptors might reveal the missing proteins connecting the complex and the fungal receptors. Apart from the downstream and upstream elements, one interesting issue will also be the three dimensional structure of the velvet complex. Co-crystalization of the complex and the effect of different light sources on the conformation of the complex elements can provide much more insight into the elaborated role of that unique protein complex. 


\section{References}

Adams, T.H., Wieser, J.K., and Yu, J.H. (1998). Asexual sporulation in Aspergillus nidulans. Microbiol Mol Biol Rev 62, 35-54.

Ballario, P., and Macino, G. (1997). White collar proteins: PASsing the light signal in Neurospora crassa. Trends Microbiol 5, 458-462.

Ballario, P., Vittorioso, P., Magrelli, A., Talora, C., Cabibbo, A., and Macino, G. (1996). White collar-1, a central regulator of blue light responses in Neurospora, is a zinc finger protein. Embo J 15, 1650-1657.

Bok, J.W., and Keller, N.P. (2004). LaeA, a regulator of secondary metabolism in Aspergillus spp. Eukaryot Cell 3, 527-535.

Braus, G.H., Krappmann, S., and Eckert, S. (2002). Sexual development in ascomycetes: Fruit body formation in Aspergillus nidulans. In Molecular Biology of Fungal Development, Osiewacz, ed (New York: Marcel Dekker Inc), pp. 215-244.

Busch, S., Schwier, E.U., Nahlik, K., Bayram, O., Helmstaedt, K., Draht, O.W., Krappmann, S., Valerius, O., Lipscomb, W.N., and Braus, G.H. (2007). An eightsubunit COP9 signalosome with an intact JAMM motif is required for fungal fruit body formation. Proc Natl Acad Sci U S A 104, 8089-8094.

Champe, S.P., Kurtz, M.B., Yager, L.N., Butnick, N.J., and Axelrod, D.E. (1981). Spore formation in Aspergillus nidulans: competence and other developmental processes. In Fungal spores: Morphogenic Controls, H.R. Hohl and G. Turian, eds (New York: Academic Press), pp. 255-276.

Dunlap, J.C., and Loros, J.J. (2004). The neurospora circadian system. J Biol Rhythms 19, 414-424.

Froehlich, A.C., Noh, B., Vierstra, R.D., Loros, J., and Dunlap, J.C. (2005). Genetic and molecular analysis of phytochromes from the filamentous fungus Neurospora crassa. Eukaryot Cell 4, 2140-2152. 
Galagan, J.E., Calvo, S.E., Cuomo, C., Ma, L.J., Wortman, J.R., Batzoglou, S., Lee, S.I., Basturkmen, M., Spevak, C.C., Clutterbuck, J., Kapitonov, V., Jurka, J., Scazzocchio, C., Farman, M., Butler, J., Purcell, S., Harris, S., Braus, G.H., Draht, O., Busch, S., D'Enfert, C., Bouchier, C., Goldman, G.H., Bell-Pedersen, D., Griffiths-Jones, S., Doonan, J.H., Yu, J., Vienken, K., Pain, A., Freitag, M., Selker, E.U., Archer, D.B., Penalva, M.A., Oakley, B.R., Momany, M., Tanaka, T., Kumagai, T., Asai, K., Machida, M., Nierman, W.C., Denning, D.W., Caddick, M., Hynes, M., Paoletti, M., Fischer, R., Miller, B., Dyer, P., Sachs, M.S., Osmani, S.A., and Birren, B.W. (2005). Sequencing of Aspergillus nidulans and comparative analysis with A. fumigatus and A. oryzae. Nature 438, 1105-1115.

Galagan, J.E., Calvo, S.E., Borkovich, K.A., Selker, E.U., Read, N.D., Jaffe, D., FitzHugh, W., Ma, L.J., Smirnov, S., Purcell, S., Rehman, B., Elkins, T., Engels, R., Wang, S., Nielsen, C.B., Butler, J., Endrizzi, M., Qui, D., Ianakiev, P., BellPedersen, D., Nelson, M.A., Werner-Washburne, M., Selitrennikoff, C.P., Kinsey, J.A., Braun, E.L., Zelter, A., Schulte, U., Kothe, G.O., Jedd, G., Mewes, W., Staben, C., Marcotte, E., Greenberg, D., Roy, A., Foley, K., Naylor, J., StangeThomann, N., Barrett, R., Gnerre, S., Kamal, M., Kamvysselis, M., Mauceli, E., Bielke, C., Rudd, S., Frishman, D., Krystofova, S., Rasmussen, C., Metzenberg, R.L., Perkins, D.D., Kroken, S., Cogoni, C., Macino, G., Catcheside, D., Li, W., Pratt, R.J., Osmani, S.A., DeSouza, C.P., Glass, L., Orbach, M.J., Berglund, J.A., Voelker, R., Yarden, O., Plamann, M., Seiler, S., Dunlap, J., Radford, A., Aramayo, R., Natvig, D.O., Alex, L.A., Mannhaupt, G., Ebbole, D.J., Freitag, M., Paulsen, I., Sachs, M.S., Lander, E.S., Nusbaum, C., and Birren, B. (2003). The genome sequence of the filamentous fungus Neurospora crassa. Nature 422, 859-868.

Green, C.B. (2004). Cryptochromes: tail-ored for distinct functions. Curr Biol 14, R847-849.

Käfer, E. (1965). Origins of translocations in Aspergillus nidulans. Genetics 52, 217-232.

Kato, N., Brooks, W., and Calvo, A.M. (2003). The expression of sterigmatocystin and penicillin genes in Aspergillus nidulans is controlled by veA, a gene required for sexual development. Eukaryot Cell 2, 1178-1186. 
Kim, H., Han, K., Kim, K., Han, D., Jahng, K., and Chae, K. (2002). The veA gene activates sexual development in Aspergillus nidulans. Fungal Genet Biol 37, 72-80.

Lin, C., and Todo, T. (2005). The cryptochromes. Genome Biol 6, 220.

Mooney, J.L., and Yager, L.N. (1990). Light is required for conidiation in Aspergillus nidulans. Genes Dev 4, 1473-1482.

Muro-Pastor, M.I., Gonzalez, R., Strauss, J., Narendja, F., and Scazzocchio, C. (1999). The GATA factor AreA is essential for chromatin remodelling in a eukaryotic bidirectional promoter. Embo J 18, 1584-1597.

Puig, O., Caspary, F., Rigaut, G., Rutz, B., Bouveret, E., Bragado-Nilsson, E., Wilm, M., and Seraphin, B. (2001). The tandem affinity purification (TAP) method: a general procedure of protein complex purification. Methods 24, 218-229.

Purschwitz, J., Muller, S., Kastner, C., and Fischer, R. (2006). Seeing the rainbow: light sensing in fungi. Curr Opin Microbiol 9, 566-571.

Sancar, A. (2000). Cryptochrome: the second photoactive pigment in the eye and its role in circadian photoreception. Annu Rev Biochem 69, 31-67.

Stinnett, S.M., Espeso, E.A., Cobeno, L., Araujo-Bazan, L., and Calvo, A.M. (2007). Aspergillus nidulans VeA subcellular localization is dependent on the importin alpha carrier and on light. Mol Microbiol 63, 242-255.

Timberlake, W.E. (1990). Molecular genetics of Aspergillus development. Annu Rev Genet 24, 5-36. 


\section{Özgür Bayram, MSc in Molecular Biology.}

Birthdate / place $\quad$ 14.05.1978 in Adapazari / Sakarya, Turkey

Education :

1983-1988 Primary education Kumbasi-Karacomaklar Primary School, Adapazari

1988-1991

Secondary education Zübeyde Hanim Secondary School, Adapazari

1991-1994 High school education Fatih Anatolian Technical, Industry and Training School, Electronics, Adapazari

Scientific Background :

$1996-2000$

2000-2001

$2001-2003$

2003-2007
Study of Biology at the Uludag University, Bursa, Turkey

Study of English Language Kings School of English, Bournemouth England

Master of Science as Research assistant at the Uludag University, Title: "The analysis of the effects of the growth conditions on the transcription and frameshift rate of Ty2 retrotransposon" in the lab of Prof. Dr. Sezai Türkel

Research assistant and Ph.D work at the Department of Molecular Microbiology \& Genetics in the group of Prof. Dr. Gerhard. H. Braus, at the Institute of Microbiology and Genetics, Georg-AugustUniversity, Goettingen, Germany 